\title{
WestVirginiaUniversity
}

THE RESEARCH REPOSITORY @ WVU

Graduate Theses, Dissertations, and Problem Reports

2010

\section{Dynamic bandgap tuning of solid thin film photonic crystal structures}

Hyma Yalamanchili

West Virginia University

Follow this and additional works at: https://researchrepository.wvu.edu/etd

\section{Recommended Citation}

Yalamanchili, Hyma, "Dynamic bandgap tuning of solid thin film photonic crystal structures" (2010). Graduate Theses, Dissertations, and Problem Reports. 4677.

https://researchrepository.wvu.edu/etd/4677

This Thesis is protected by copyright and/or related rights. It has been brought to you by the The Research Repository @ WVU with permission from the rights-holder(s). You are free to use this Thesis in any way that is permitted by the copyright and related rights legislation that applies to your use. For other uses you must obtain permission from the rights-holder(s) directly, unless additional rights are indicated by a Creative Commons license in the record and/ or on the work itself. This Thesis has been accepted for inclusion in WVU Graduate Theses, Dissertations, and Problem Reports collection by an authorized administrator of The Research Repository @ WVU. For more information, please contact researchrepository@mail.wvu.edu. 


\title{
DYNAMIC BANDGAP TUNING OF SOLID THIN FILM \\ PHOTONIC CRYSTAL STRUCTURES
}

\author{
by
}

\section{Hyma Yalamanchili}

\author{
Thesis submitted to the \\ College of Engineering and Mineral Resources at \\ WEST VIRGINIA UNIVERSITY \\ in partial fulfillment of the requirements for the degree of \\ Master of Science \\ in \\ Electrical Engineering
}

Committee members:

Dr. Jeremy M. Dawson, Committee Chairperson

Dr. Lawrence A. Hornak

Dr. Dimitris Korakakis

Lane Department of Computer Science and Electrical Engineering

\section{Morgantown, West Virginia}

2010

Keywords: Photonic Crystals, E-beam Lithography, Bandgap tuning, Reflectivity, Ellipsometry, ANSYS, Aluminum Nitride, MPB 


\title{
Dynamic Bandgap Tuning of Solid Thin Film Photonic Crystal Structures
}

\author{
Hyma Yalamanchili
}

\begin{abstract}
Nanophotonics, specifically photonic crystals (PhCs), offer unique optical bandgap engineering possibilities that has driven the emergence of a variety of device platforms, including: beam splitters, nano-cavity resonators, lasers, fibers, waveguides and highly sensitive optofluidic biosensor devices. The design and fabrication of accurate lattice parameters for a $\mathrm{PhC}$ is very important to achieving the desired operating bandgap. The inclusion of tunability in thin film PhCs not only offers a means of adjusting for fabrication errors but also a mechanism to increase device functionality as well as providing a wider range of operating wavelengths. Nitride thin films, specifically Aluminum Nitride (AlN) and Gallium Nitride ( GaN), are being used as PhC slab materials by our group due to their desirable optical properties at visible wavelengths and high chemical and thermal stability under harsh conditions. The inherent piezoelectric properties of these materials offer a means of direct tuning of $\mathrm{PhC}$ lattice parameters through piezoelectric deformation.
\end{abstract}

This thesis presents the results of research aimed at actively tuning the bandgap of PhCs fabricated in piezoelectric AlN thin films. Theoretical investigations of the bandgap tuning of 'as-drawn' and deformed 1- and 2-D PhC lattice structures using coupled results from $\mathrm{PhC}$ optical behavioral modeling and finite element mechanical simulations are discussed. The results of experimental characterization of the optical and mechanical (i.e. tuning) properties of micro to nanoscale PhC lattice structures fabricated in Si and AlN using e-beam and optical lithography and reactive ion etching are presented. Experimental data is then used to explore the bandwidth tuning capability of large-area periodic nanophotonic structures. 


\section{Acknowledgements}

I would like to express my sincere thanks to Dr. Jeremy Dawson, my research advisor and committee chairperson who has been a constant source of motivation and support throughout my research work. I would also like to thank him for helping me to develop wide range of skills in design, fabrication as well as in characterization processes. I bestow my sincere thanks to my committee members Dr. Lawrence Hornak and Dr. Dimitris Korakakis for their time, valuable suggestions and support throughout my research experience. I would like to acknowledge Dr. Kolin Brown for his assistance and guidance in the clean room. I would like to express my special thanks to Sridhar Kuchibhatla and Henry Andagana for their constant support and informative discussions. I would also like to thank Swetha Sanju Koduri, Bashar Hamza and Anand Kadiyala for their support throughout my work.

Most importantly, I would like to thank my father, Y.V. Padmanabham and my mother, Sridevi for their unconditional love and support without which nothing would have been possible in my life. I would also like to thank my elder sister Swapna for her love and care towards me all these years. Last but not least, I would like to thank my fiancé, Naveen Kantamneni for his love, emotional support and great understanding throughout my research work. He always stood by me in my hardships. I look forward to spending rest of my life with him. I dedicate my thesis to my parents, sister and my fiancé. 


\section{Table of Contents}

CHAPTER 1: Introduction to Photonic Crystals and Tuning .................................................... 1

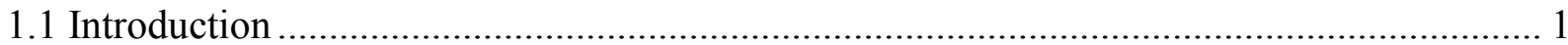

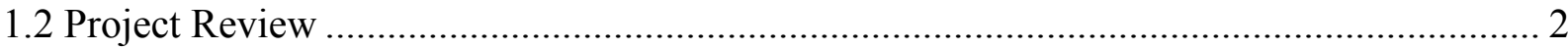

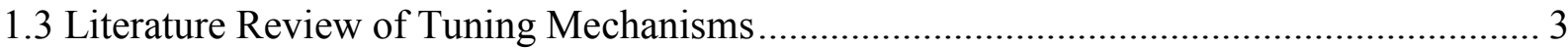

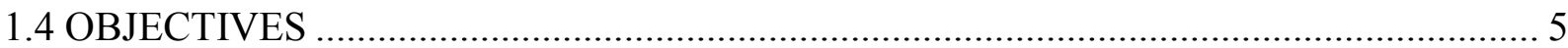

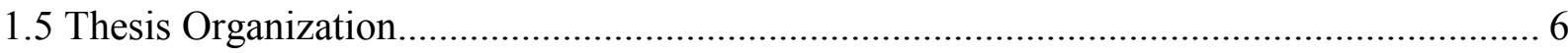

CHAPTER 2: THEORY .......................................................................................................................... 7

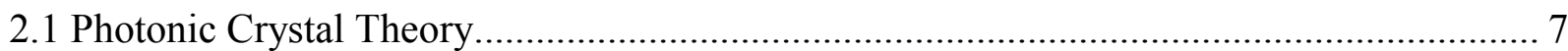

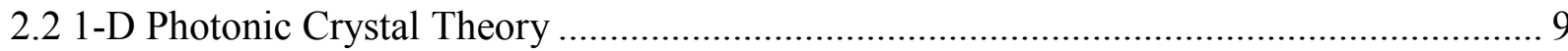

2.3 Photonic Crystal Slab and Off-Axis Propagation .............................................................. 11

2.3 Piezoelectric properties of Nitride Thin Films ............................................................. 12

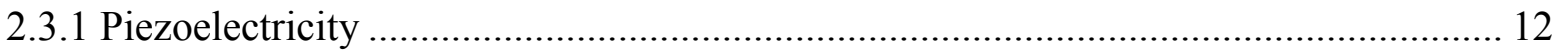

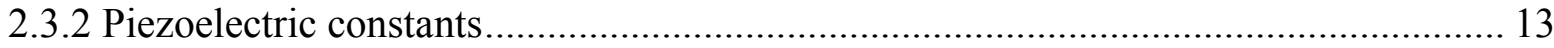

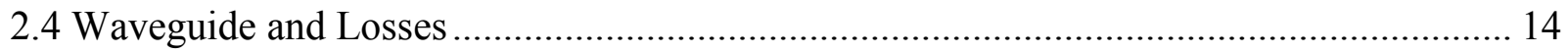

CHAPTER 3: THEORETICAL MODELING ....................................................................... 17

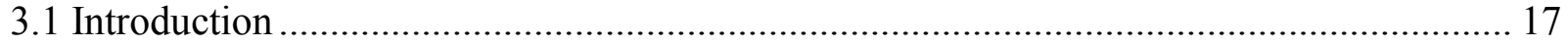

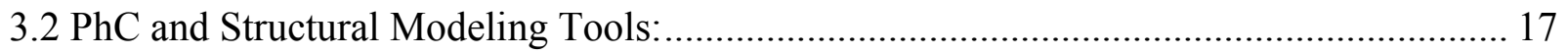

3.2.1 MPB and FEA of 2-D Photonic Crystal ................................................................ 17

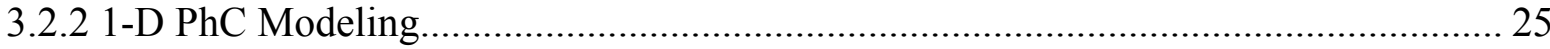

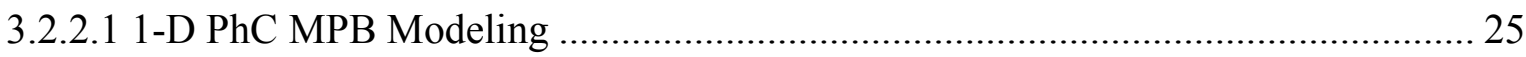

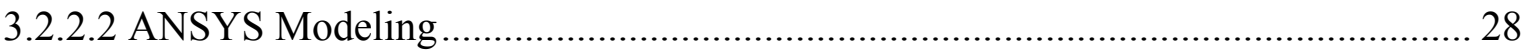

3.2.2.2(a) DC Analysis and Harmonic Analysis of 1-D PhC:_.................................. 28

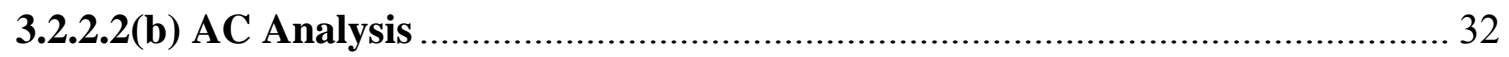

3.3 MPB Modeling for Polycrystalline PhC structures in $\mathrm{GaN}$................................................ 33

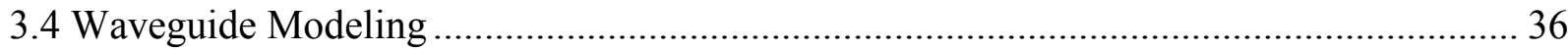

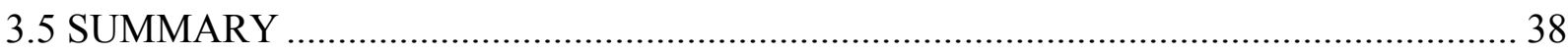

CHAPTER 4: FABRICATION ................................................................................................ 39

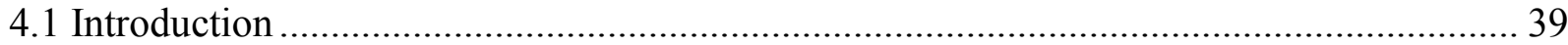

4.2 Fabrication of 1-D PhCs for optical characterization....................................................... 39 


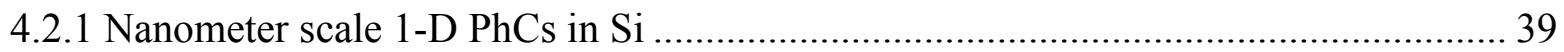

4.2.2 Introduction to Aluminum Nitride:.................................................................... 41

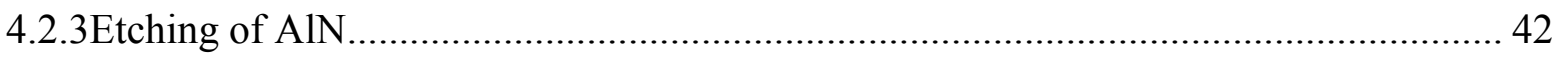

4.2.4 Nanometer scale 1-D PhCs in AlN ..................................................................... 42

4.3 Fabrication for Piezoelectric Characterization ........................................................ 47

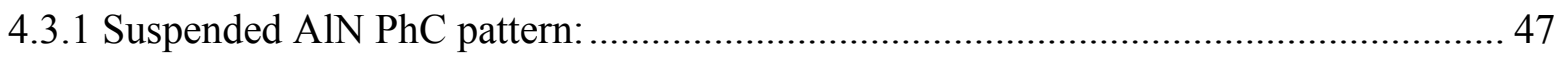

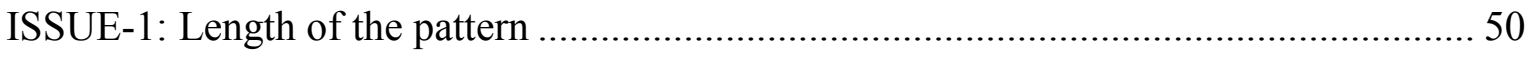

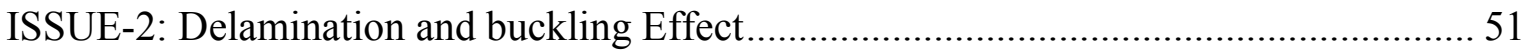

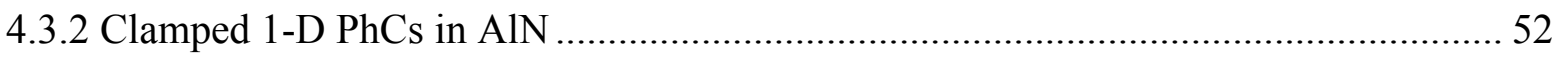

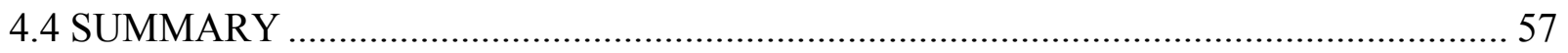

CHAPTER 5: CHARACTERIZATION........................................................................ 58

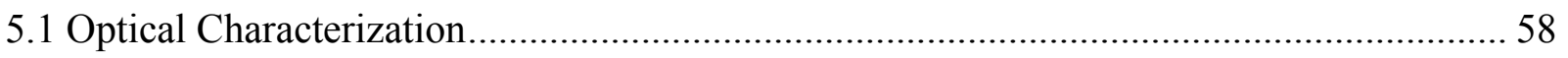

5.2 Photonic Crystal Characterization......................................................................... 58

5.2.1 Band Diagram Characterization Techniques using Normal Incidence ...................... 58

5.2.2Band diagram Characterization using Surface Coupling Technique.......................... 60

5.1.2.1 Earlier Work in Surfacing coupling Technique................................................ 62

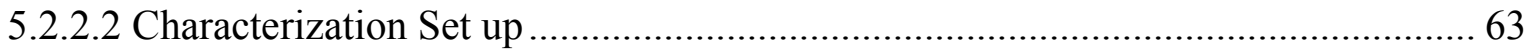

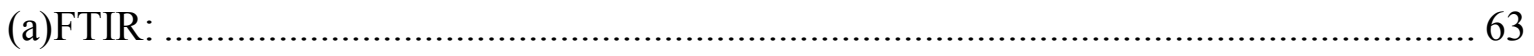

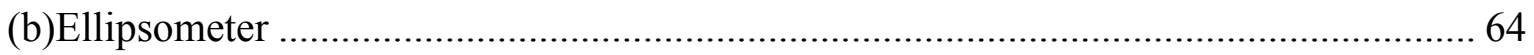

5.3 Optical Characterization of Waveguides................................................................... 71

5.3.2 Prism Coupling Characterization: .................................................................. 71

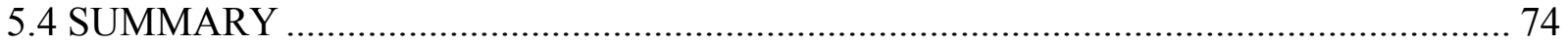

Conclusions and Future Work....................................................................................................... 75

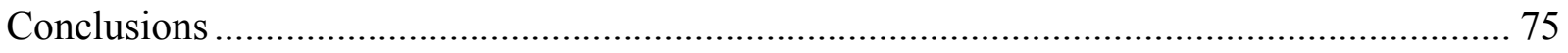

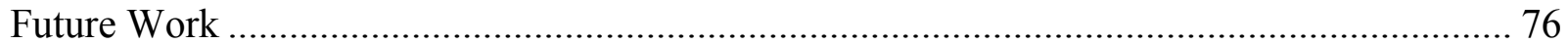

APPENDIX A: PRISM COUPLER OPERATION ............................................................ 77

APPENDIX B: Diffraction Grating Theory ................................................................................... 78

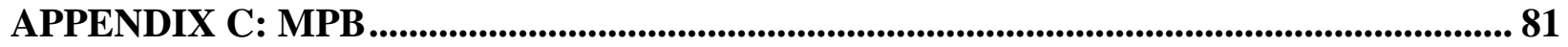

APPENDIX D: ELLIPSOMETRY/Reflectivity .................................................................. 84

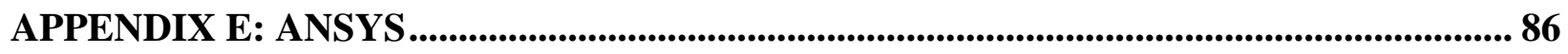

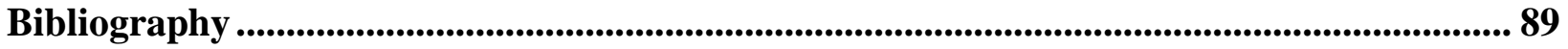




\section{List of Figures}

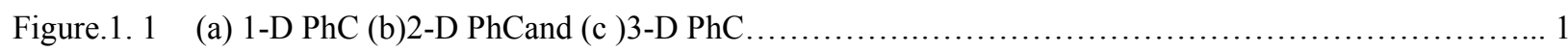

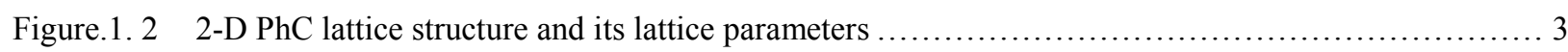

Figure. 2.1 TE Band diagram of triangular lattice of air $(\mathrm{n}=1)$ holes in $\mathrm{Si}(\mathrm{n}=3.45)$ with radius $0.28 \mathrm{a}$ has a gap-to-

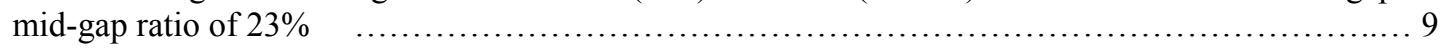

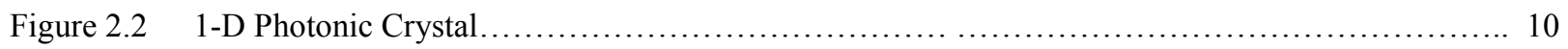

Figure 2.3 Band diagram of a 1-D Photonic crystal with off-axis propagation................................11

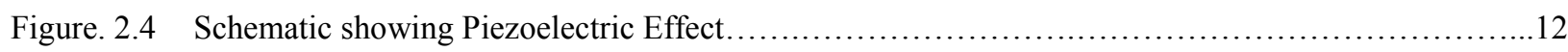

Figure. 2.5 Waveguide profile showing total internal reflection.........................................

Figure3.1 TM Band Diagram of 2-D PhC with triangular lattice of air holes in AlN with a radius of 0.29a has a gap-to-mid gap ratio of $21 \%$ between $1^{\text {st }}$ and $2^{\text {nd }}$ bands and $7 \%$ between $3^{\text {rd }}$ and $4^{\text {th }}$ bands............18

Figure3.2 TE-like or zeven band diagram of 2-D PhC in AlN slab with a gap-to-midgap ratio of $10 \%$ between the first two guided bands under the light cone. 19

Figure3.3 Band Diagram of 2-D Phc in AlN slab with a common band gap of $\sim 9.8 \%$ between the guided modes

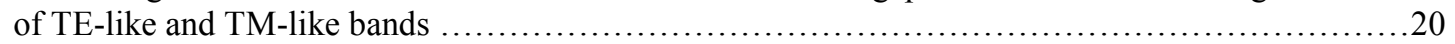

Figure3.4 FEA of 2-D PhC showing the displacement in $\mathrm{x}$-direction when the edges of the slab are clamped... 23

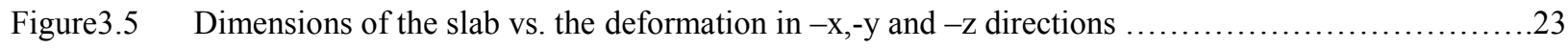

Figure3.6 Voltage applied to the slab vs. deformation in $-\mathrm{x},-\mathrm{y}$ and $-\mathrm{z}$ direction................................... 24

Figure3.7 Deformation of 2-D PhC in $-\mathrm{x}$ direction when one side of the slab is clamped ..................... 24

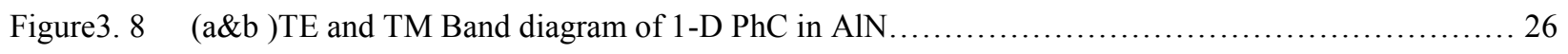

Fig. 3.9 Width of the air grating vs. gap-to-midgap ratio of 1-D PhC in AlN .............................27

Figure3.10 Slab thickness of 1-D PhC in AlN bounded by air cladding vs. gap-to midgap ratio................. 27

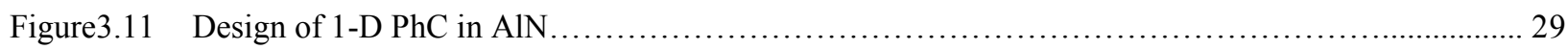

Figure3.12 Deformation vs. voltage for a model which gives minimum deflection......................... 29

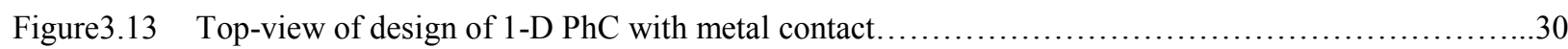

Figure3.14 Z-Deflection of 1D PhC with application of $10 \mathrm{~V}$ on the 1-D PhC with electrode. .................... 31

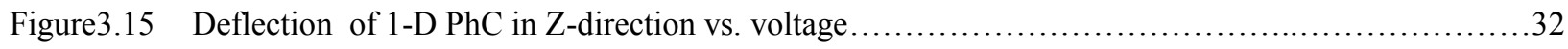

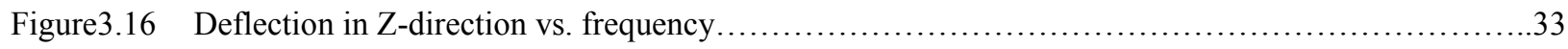

Figure3.16 (a)Lattice domain with 7X9arrays of holes (b)lattice domain with 13 X17 arrays of holes (c)17X29

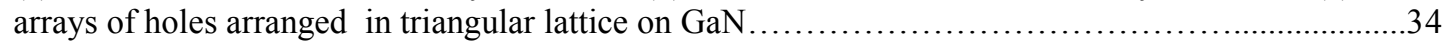




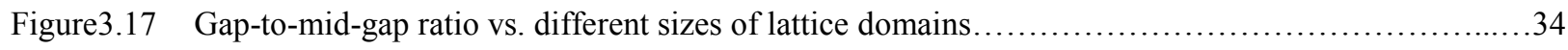

Figure3.18 Bandgap vs. angle of incidence (variation of K points) ..................................... 35

Figure3.19 (a,b,c,d) Adjacent domains placed at an angle of $45^{\circ}$ to each other with different lattice domain sizes (e) Gap-to-midgap ratio vs. different lattice domains sizes of adjacent PhCs placed at an angle of $45^{\circ}$ to

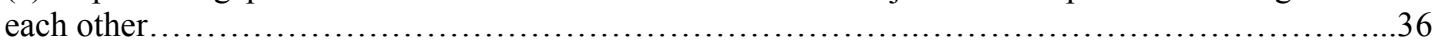

Figure3.22 (1)GaN waveguide on top of a thin AlN layer and sapphire substrate. (2)The field intensity as modeled by OptBPM that shows a complete confinement within the waveguide (3) Field intensity (green) plotted across the different refractive indices of the layers (red.) .37

Figure3.23 (1)The GaN waveguide with an underlying $\mathrm{SiO}_{2}$ layer which will eventually be removed after the fabrication process is finished and obtain a flow channel instead. (2) The field intensity demonstrates the confinement of the light in the waveguiding GaN layer that will include the PhC structure. (3) Field intensity (green) plotted across the different refractive indices of the layers (red ) ...............38

Figure 4. 1 Process flow for fabricating nanometer scale 1-D PhCs in Si and SOI...............................39

Figure 4. 2 1-D PhC pattern with 700nm wide Si stripe with 300nm air spacing on SOI samples............... 40

Figure 4. 3 1-D PhCs pattern with 400nm wide Si stripe with 300nm air spacing on SOI samples. Samples were

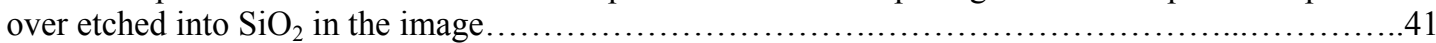

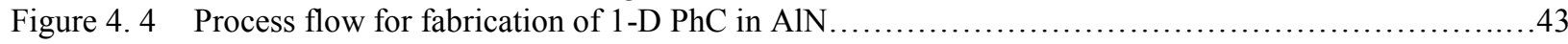

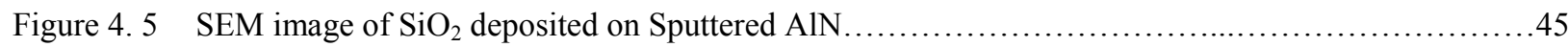

Figure 4.6 1-D PhC patterns in E-beam resist spun on AlN after development for $35 \mathrm{sec} \ldots \ldots \ldots \ldots \ldots \ldots \ldots \ldots \ldots . \ldots 45$

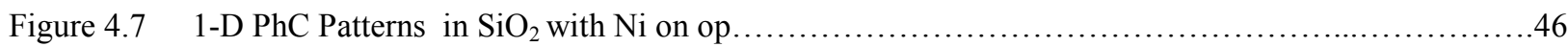

Figure 4.8 1-D PhC patterns in AlN etched into Si.........................................................

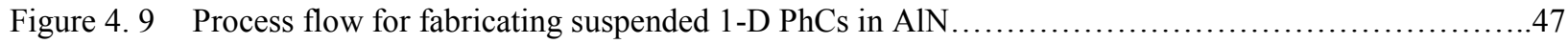

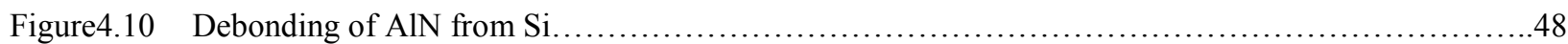

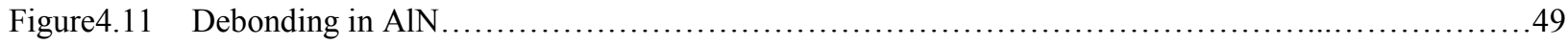

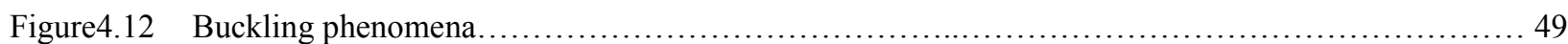

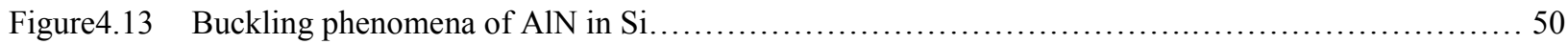

Figure4.14 Debond from an edge of the film, driven by residual tensile stress ${ }^{11}$ (Courtesy of Q. Ma of Intel

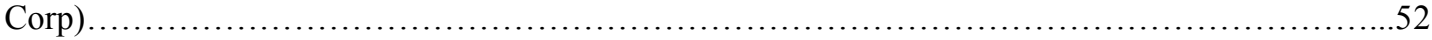

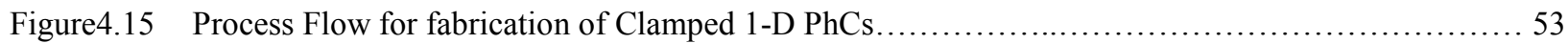

Figure4.16 SOI samples with 1-D PhCs transferred into $\mathrm{Si}\left(1.5 \mu \mathrm{mSi} / 2.5 \mu \mathrm{mSiO}_{2}\right)$ after ICP/RIE etch..........54

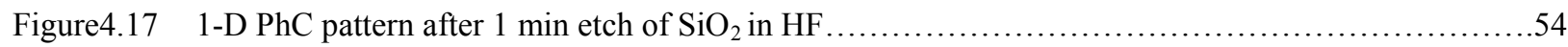

Figure4.18 Side view of 1-D PhC patterns in Si suspended or released from substrate ..........................55

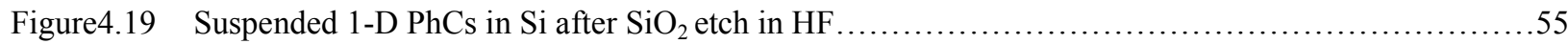

Figure4.20 Clamped 1-D PhCs after AlN deposition on suspended Si PhC patterns followed by Pt-image 01.....56

Figure4.21 Clamped 1-D PhCs after AlN deposition on suspended Si PhC patterns followed by Pt-image02 .......56

Figure4.22 (a\&b)Composition of $\mathrm{Al} \& \mathrm{~N}_{2}$ in Sputtered $\mathrm{AlN}$ on $\mathrm{Si}$ and SOI wafers respectively................. 57

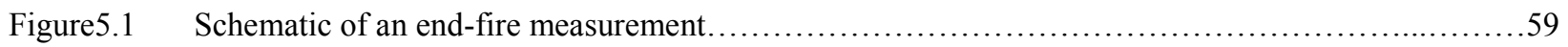


Figure5.2 (a) Schematic of ILS experiment (b) side-view of schematic (c) Typical experimental

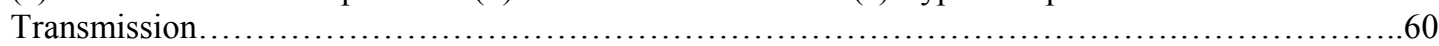

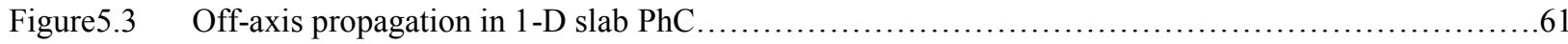

Figure5.4 Experimental geometry of 1-D and 2-D structures used by Astratov et.al...........................62

Figure5.5 Angular dependent TM polarized reflectivity spectra from a 2-D honeycomb lattice of air cylinders

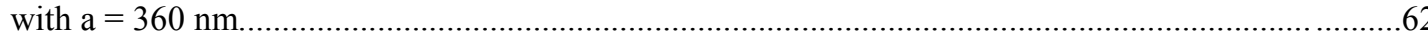

Figure5.6 TE and TM polarized spectra mapped on to the 2-D model of perfectly confining waveguide model

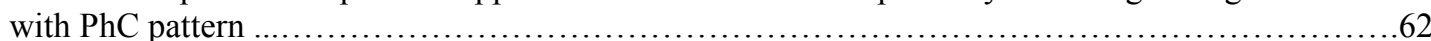

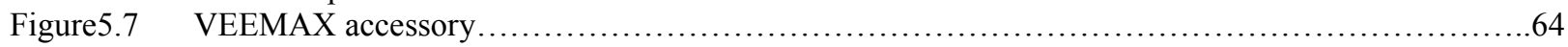

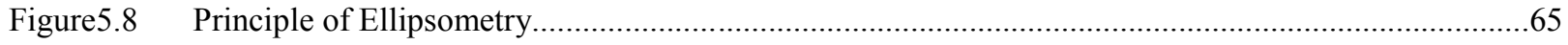

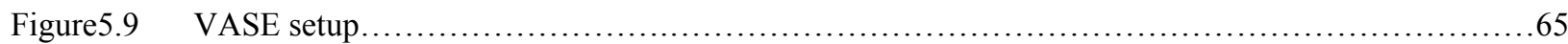

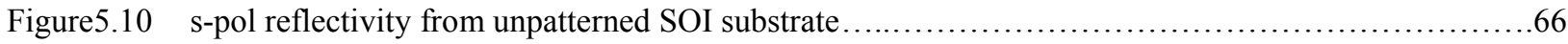

Figure5.11 s-pol reflectivity spectra of 1-D PhC in SOI $\left(700 \_300 \mathrm{~nm}\right)$ wafers............................67

Figure5.12 Theoretical and experimental data from the surface coupling technique plotted...................67

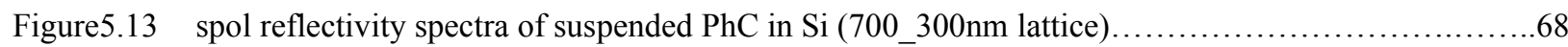

Figure5.14 zeven band diagram and experimental data from 1-D PhC suspended in air with lattice constant of 1

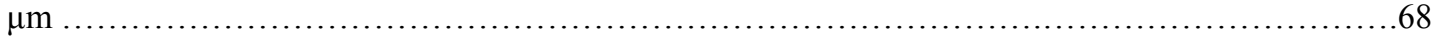

Figure5.15 spol and ppol reflectivity spectra of 1-D PhC suspended in air in 400_200nm lattice respectively...70

Figure5.16 zeven and zodd Band Diagram of 1-D PhCs in Si suspended in air with lattice constant 600nm......70

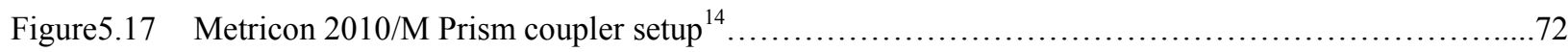

Figure5.18 Cross -sectional view of the prism coupler layout............................................. 73

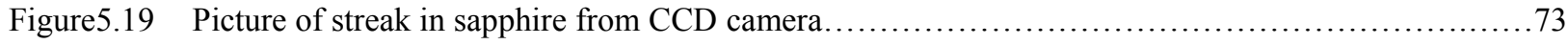

Figure5.20 (a) The guiding streak through the GaN slab. (b) The plotted intensity decay across the propagating

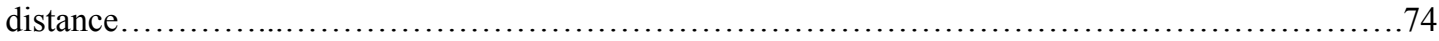




\section{CHAPTER 1: Introduction to Photonic Crystals and Tuning}

\subsection{Introduction}

Photonic crystals $(\mathrm{PhCs})^{1}$ are engineered nanostructures exhibiting one, two, or three dimensional periodicity of refractive index as shown in Figure 1.1. PhCs provide unique optical properties for controlling and manipulating the flow of light. Similar to the electrons in a crystalline semiconductor, photons are scattered by a crystal of periodically arranged dielectric. Photon propagation through such nanostructures depends on the varying refractive index which creates a photonic band-gap, i.e. a region in which propagation of light is prohibited.

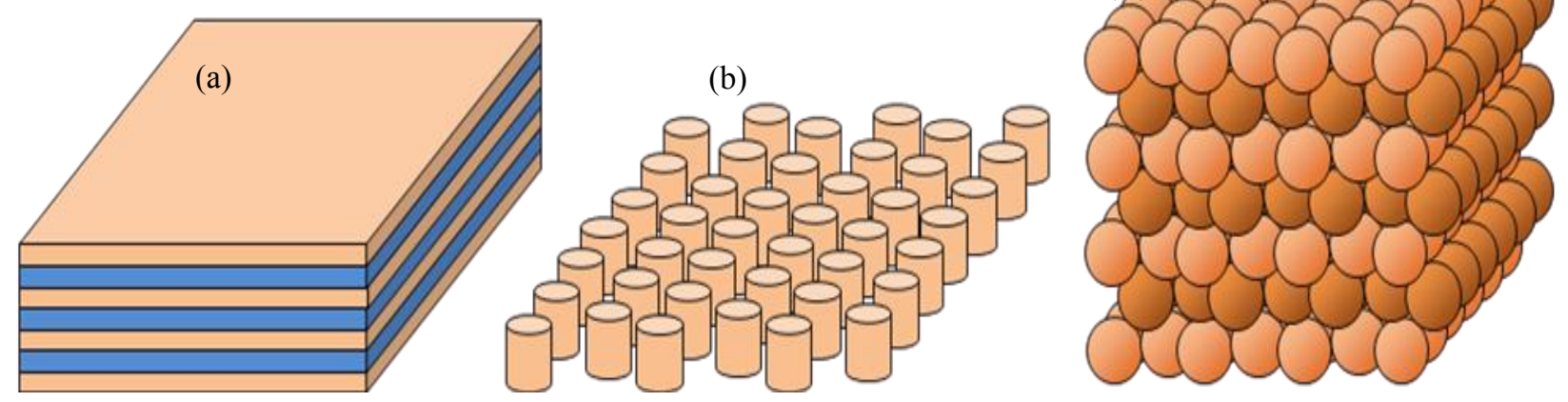

Figure1. 1 (a) 1-D PhC (b)2-D PhCand (c )3-D PhC

The ability to engineer the bandgap of PhCs is central to the realization of many device applications such as waveguides, beam splitters, nanocavity resonators, lasers, LEDs, biosensors e.t.c. A brief explanation of few PhC applications is discussed below.

\section{Applications}

$P h C L E D s^{2}$ : For typical semiconductor LEDs, a large fraction of the energy is emitted into waveguided modes internal to the semiconductor rather than radiation modes. Light generated inside the semiconductor bounces around due to total internal reflection, and there is a high probability that the light will be absorbed before it can escape from the semiconductor. A photonic crystal can improve light extraction by diffracting waveguided modes out of the semiconductor.

Nanocavity Lasers $^{3}:$ PhCs with engineered defect cavities can have extremely high quality factors to modal volume ratios. These ratios are proportional to the spontaneous emission rate in a micro-cavity. Before the widespread use of photonic crystals, the advantages of large spontaneous rate enhancement could not be fully explored in lasers because of their large mode volumes. With the recent advances in the fabrication of high quality PhCs in semi- 
conductors, ultrafast, efficient and compact lasers which show great promise can be achieved with a defect in PhCs for applications in high -speed communications, information processing and on-chip optical interconnects.

Beam Splitters ${ }^{4}$ : Defects into photonic bandgap structures can be used to manipulate photons in highly localized regions. There are different kinds of defects such as line defects which can be used to realize straight or bent waveguides. Furthermore, point defects can be introduced into line defects to build wavelength add-drop devices or to improve the transmission properties of bent waveguides. Combinations of line defects such as T-, Y-, and crosstype waveguides can act as photonic crystal beam splitters.

Sensors $^{5}$ : Sensing schemes based on photonic crystals $(\mathrm{PhCs})$ are very well suited for integration, because they are compact and have tunable properties. Due to their unique optical properties, PhCs are emerging as platforms for optical bandgap engineering in opto-fluidic systems as low limit of detection transducer for biosensing applications. One such sensor element under development by our group employs a thin film 2-D PhC which acts as a transducer that possesses an engineered lattice defect ${ }^{6,7,8}$ as shown in Figure 1.2. The resonant property of the lattice defect leads to confinement of photons emitted from the flouroscent labels which shows potential for increasing the detection limit of fluoreescence spectrocsopy techniques.

\subsection{Project Review}

Though 3-D PhCs offer a confinement of light in all the three directions, top-down fabrication of 3-D PhCs is difficult. This makes them less viable for use in device applications. Thin film PhCs, which are essentially 1- or 2-D $\mathrm{PhCs}$ fabricated on thin films with a finite thickness, are commonly employed in $\mathrm{PhC}$ device architectures due to their unique optical properties and their ease in fabrication.

A major hurdle in realizing thin-film $\mathrm{PhC}$ device structures optimized for visible wavelengths is that they invariably require critical dimensions that are often on the order of 100-300 nm, making reliable, accurate transfer of ideal model parameters to fabricated structures particularly challenging. In this respect, critical dimension variations as small as $5-7 \%$ is sufficient to dramatically shift the photonic bandgap and severely alter or disallow proper device functionality. For instance if a $\mathrm{PhC}$ is designed with a defect to exhibit resonance at a particular wavelength, and if the fabricated lattice parameters are different compared to designed values, then the $\mathrm{PhC}$ structure does not exhibit 


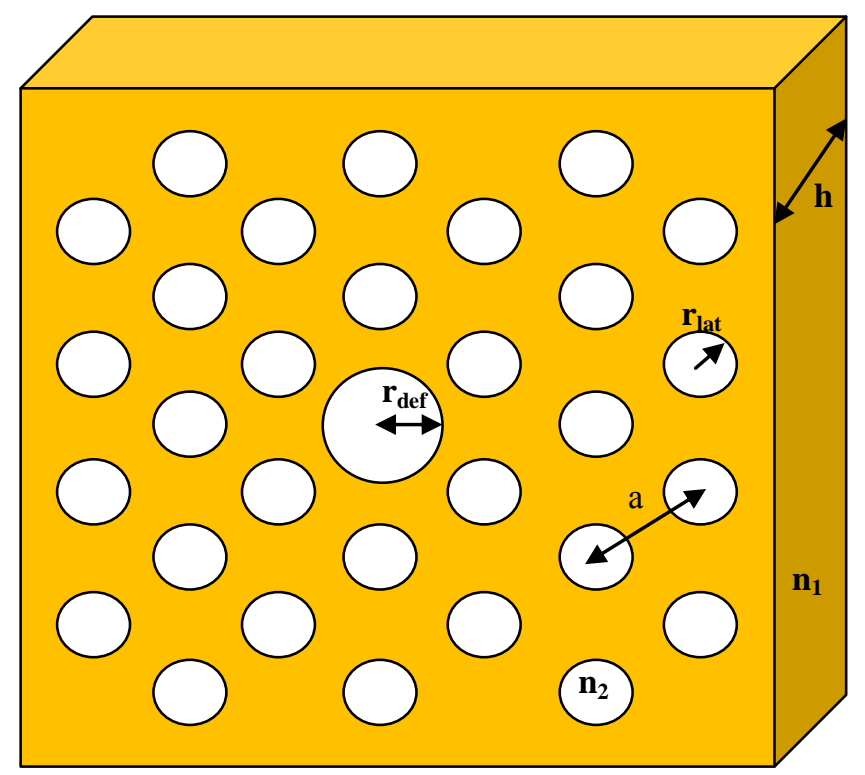

Figure1. 2 2-D PhC lattice structure and its lattice parameters ${ }^{8}$

resonance at desired value which will eventually alter the functionality of the device. As a consequence, the challenges associated with fabricating thin-film photonic crystals are barriers that, to date, have significantly limited their practical utility in biophotonics, advanced imaging, and sensing applications, namely: fixed device characteristics after fabrication and nanofabrication process variations that lead to decreased device performance and yield.

The addition of parameter tuning to $\mathrm{PhC}$ devices through well-designed and direct in situ piezoelectric material deformation and rearrangement of the $\mathrm{PhC}$ lattice will not only provide the performance and reliability improvements but also potentially provide a wide operation range for a $\mathrm{PhC}$. Photonic bandgap tuning of a specific PhC structure can be achieved through various techniques. A few techniques presented in the literature are discussed in the next section.

\subsection{Literature Review of Tuning Mechanisms}

Tuning mechanisms applied to PhCs depend largely on the structural geometries and the desired operating wavelengths. The $\mathrm{PhC}$ tuning methods reported in the literature can be classified into two distinct groups:

1. Post-Fabrication Tuning: uses micro-and nano fabrication methods to tune the geometrical variations; referred to in this thesis as static tuning.

2. In-Situ Tuning: varying the PhC structure during device implementation achieve the improved or variable operation of the device; referred to in this thesis as dynamic tuning. 
The latter mechanism of tuning can further be grouped by different tuning techniques applied to affect different properties of PhCs. These dynamic tuning techniques include:

(i)Tuning by Temperature ${ }^{9,10}$ : H.Nemec et.al ${ }^{11}$ fabricated Bragg mirrors with alternating layers of quartz and high permittivity ceramics with a defect layer of $\mathrm{SrTiO}_{3}$. The defect layer which exhibits ferro-electric properties was tuned thermally for a single defect mode over the entire forbidden band. They achieved a tuning of almost $60 \%$ for this structure in the tetrahertz range

(ii)Tuning by Magnetic field ${ }^{2}$ : Sergey Savel'ev et.al fabricated a Josephson vortex (JV) lattice which is a periodic array of layered superconductors and insulators that scatters Josephson plasma waves. This produces a photonic band gap structure in terahertz frequency range. The gaps were easily controlled or tuned by either the in-plane magnetic field or the transverse transport current flowing across the superconducting layers. They proved that a small change in the in-plane component of magnetic field can switch the sample from fully transparent to fully reflective within given frequency windows. Thus, the material can change from a tetra hertz glass to a mirror by merely changing in-plane magnetic field or transport current. Magneto-optical Voigt effect has been used to vary the negative refractive index of $n$-doped GaAs photonic crystals by Liang Feng et.al ${ }^{13}$. Tunability of super paramagnetic PhCs self-assembled in colloidal magnetic fluids was achieved using external magnetic field by Shengli Pu $\boldsymbol{e t . a l}^{14}$. The band structures of 2-D PhCs of triangular lattice with limited heights of magnetic columns were calculated and the simulation results indicated that band gaps of z-odd modes can be easily tuned by external magnetic field while zodd modes have a weak dependence on external magnetic field.

(iii) Tuning by AFM: Lalouat et.al $l^{15}$ and I. Marki $e t . a^{16} l$ used sub-wavelength AFM tips to tune the resonance of the defect cavity of PhCs.

(iv)Tuning by optical-methods: Different optical-methods were used to tune or control PhC operation, which include external pump laser excitation of free carriers for resonance tuning ${ }^{17,18}$, cavity resonance tuning using a laser to cause localized thermal and plasma dispersion ${ }^{19}$, and photosensitive materials usage in the fabrication of PhC lattice structures $^{20}$. Liquid-crystal material addition to the 1-D PhC structures of alternating layers of air and dielectric was used for tuning the refractive index of the air region which in, turn, tunes the bandgap of $\mathrm{PhC}^{21,22,23}$, as well as the The same was performed by controlled addition \& extraction of other gas- and liquid-phase materials ${ }^{24,25}$.

(v) Tuning by mechanical methods: MEMS and NEMS actuators were attached to the Si gratings to achieve structural deformation to tune the bandgap of $\mathrm{PhCs}^{26,27,28,29,30,31}$. In this technique tuning of the bandgap depends 
directly on the variation in the geometrical properties of the $\mathrm{PhC}$ in $\mathrm{Si}$ which in turn depends on the piezoelectric deformation caused by the MEMS actuators. A tunable photonic bandgap micro cavity has been designed, fabricated and characterized to operate at $1.55 \mu \mathrm{m}$ wavelengths using piezoelectric actuators on silicon, indicating that piezoelectric strain tuning provides significantly faster response and better localization of tunability ${ }^{32}$. In this referenced work, the diffracted angular change, $\Delta \theta$, is related to the grating period change, $\Delta d$, through,

$$
\Delta \theta \cong \frac{m \lambda \Delta d}{d^{2}}
$$

where $m$ is the diffracted order, $\lambda$ is the wavelength, and $d$ is the grating period.

PhCs were fabricated in nitride thin-films specifically Aluminum Nitride (AlN) and Gallium Nitride (GaN) were chosen as $\mathrm{PhC}$ substrates by our group because of the operating spectrum (visible) of our sensor architectures, their high chemical and mechanical stability under harsh conditions, low loss in the visible spectral range as well as high indices of refraction, which is necessary to obtain a wide photonic bandgap and better light confinement within their defects. These films also exhibit piezoelectric properties at elevated temperatures which can potentially be employed for direct bandgap tuning. Instead of using actuators to tune the geometry of Si gratings as discussed in literature ${ }^{36}$, piezoelectric properties of nitride thin films in which PhCs were fabricated can potentially be used to directly tune the bandgap. The application of an electric field induces strain on the $\mathrm{PhC}$ structure in piezo-thin films that deforms it to allow for direct tunability of the bandgap.

\subsection{OBJECTIVES}

As discussed, fabrication errors result in alteration of device functionality as $\mathrm{PhC}$ designs are transferred from ebeam writing files to actual device structures. In addition, thin-film PhCs possess fixed optical properties that cannot be dynamically changed after fabrication is complete. To overcome the challenges imposed by fabrication errors and limitations of fixed lattice geometries in thin-film PhCs, this research explores the direct bandgap tuning capabilities of thin-film nitride PhCs. The main objectives of this research are:

(a) To Design and simulate 2-D/ 1-D PhCs using MPB modeling tool.

(b) To calculate the losses imposed by the PhC slab structure by initially modeling waveguides using OptiBPM.

(c) Modeling of 1-D and 2-D PhCs in AlN using ANSYS to analyze the piezoelectric tuning capabilities.

(d) Fabrication of nanometer scale 1-D PhCs in Si and AlN for optical characterization. 
(e) Fabrication of micron-scale 1-D PhCs in AlN for piezoelectric characterization.

(f) Optical Characterization of 1-D PhCs using ellipsometer and Waveguide characterization using Prism coupler setup.

(g) Mechanical Characterization of micron-scale 1-D PhCs using Vibrometer.

\subsection{Thesis Organization}

The material presented in this these details efforts toward meeting the goals stated in the previous section. Chapter 2 discusses the optical theory for PhCs and the proposed piezoelectric tuning mechanism. Chapter 3 presents the results of PhC modeling efforts using MPB modeling and piezoelectric modeling using ANSYS. It also includes a discussion of waveguide modeling efforts using Opti-BPM software to determine the optimum GaN slab thickness for light guiding. Fabrication processes development efforts for nano- and micro-meter scale 1-D PhCs are presented in Chapter 4. Chapter 5 presents characterization techniques used to experimentally measure PhC optical and piezoelectric properties. It outlines the results acquired by ellipsometric characterization of PhCs and presents prism coupler characterization of asymmetric slab waveguides. 


\section{CHAPTER 2: THEORY}

\subsection{Photonic Crystal Theory}

Photonic crystals are optical analogs to semi-conductor materials in which there exists a complete bandgap between valence and conduction bands. The propagation of light in a periodic dielectric media is regulated by four Maxwell's Equations ${ }^{1}$.

$$
\begin{gathered}
\nabla \cdot B=0 \\
\nabla \cdot D=\rho \\
\nabla X E+\partial B / \partial t=0, \\
\nabla X H-\partial D / \partial t=J .
\end{gathered}
$$

where $\boldsymbol{E}$ and $\boldsymbol{H}$ are electric and magnetic fields respectively, $\mathbf{D}$ is the displacement field $\left(\boldsymbol{D}=\varepsilon_{0} \boldsymbol{E}+\boldsymbol{P}\right.$, where $\boldsymbol{P}$ is the polarization density of the material and $\boldsymbol{\varepsilon}_{\boldsymbol{0}}$ is the vacuum permittivity), $\boldsymbol{H}$ is the magnetic field strength $(\boldsymbol{B}=\boldsymbol{\mu} \boldsymbol{H}$ where $\boldsymbol{\mu}$ is the magnetic permeability), $\boldsymbol{\rho}$ and $\boldsymbol{J}$ represents free charges and currents densities respectively. In a homogenous dielectric medium with no sources of light we can set $\boldsymbol{\rho}=\mathbf{0}$ and $\boldsymbol{J}=\mathbf{0}$. In a homogenous, isotropic and transparent material without dispersion, $D(r)=\epsilon_{0} \epsilon(r) E(r), B=\mu_{0} H$ and $n=\sqrt{\mu \epsilon}$, then Maxwell's equations can be simplified as

$$
\begin{gathered}
\nabla \cdot H(r, t)=0 \\
\nabla \cdot[\epsilon(r) E(r, t)]=0 \\
\nabla X E(r, t)+\mu_{0} \frac{\partial H(r, t)}{\partial t}=0 \\
\nabla X H(r, t)-\varepsilon_{0} \varepsilon(r) \frac{\partial E(r, t)}{\partial t}=0
\end{gathered}
$$

Because of the linearity of Maxwell's equations, time dependence can be separated from the spatial dependence by expanding the fields in to a set of harmonic modes. Electric field and magnetic field can be simplified as

$$
\begin{gathered}
E(r, t)=E(r) e^{-i \omega t} \\
H(r, t)=H(r) e^{-i \omega t} .
\end{gathered}
$$

To find the equations governing the mode profiles for a given frequency, inserting the above equations into $\operatorname{Eqn}(2 .(5,6,7,8))$, the two divergence equations gives the conditions

$$
\begin{gathered}
\nabla \cdot H(r)=0 \\
\nabla \cdot[\varepsilon(r) E(r)]=0 \quad .
\end{gathered}
$$


These equations indicate that there are no point sources or sinks of displacement and magnetic fields in the medium. For transverse electromagnetic fields the two curl equations relate $E(r)$ to $H(r)$ as

$$
\begin{aligned}
\nabla X E(r)-i \mu_{0} \omega H(r) & =0 \\
\nabla X H(r)+i \omega \varepsilon_{0} \varepsilon(r) E(r) & =0
\end{aligned}
$$

Upon simplification of equations 2.13 and $2.14\left(c=1 / \sqrt{\varepsilon_{0} \mu_{0}}\right)$, the resulting equation in $H(r)$ is given by ${ }^{1}$

$$
\nabla \times\left(\frac{1}{\varepsilon(r)} \nabla \times H(r)\right)=\left(\frac{\omega}{c}\right)^{2} H(r)
$$

This equation is known as the master equation which can be solved to acquire the modes of $H(r)$ and the corresponding frequencies. $E(r)$ can then be determined by substituting $H(r)$ into Eqn (2.13).

Eqn 2.15 gives a result which will be the original function multiplied by a constant. This is an example of an eigen value problem in mathematical physics where the constant is known as eigen value and the function $H(r)$ is known as an Eigen function.

One of the most important implications of equation 2.15 is the scaling property. Suppose we scale the photonic crystal structure by a factor of $s$, so that,

$$
\epsilon^{\prime}(r)=\epsilon(r / s)
$$

Then, by performing a change of variable with $r^{\prime}=s r$ and, $\nabla^{\prime}=\nabla / s$, equation 2.15 becomes,

$$
\nabla^{\prime} X\left(\frac{1}{\epsilon^{\prime}\left(r^{\prime}\right)} \nabla^{\prime} X H\left(\frac{r^{\prime}}{s}\right)\right)=\left(\frac{\omega}{c s}\right)^{2} H\left(r^{\prime} / s\right)
$$

We get the same equation as 2.15 with eigenvector $H(r)=H\left(r^{\prime} / s\right)$ and eigenvalue $\omega^{\prime}=\omega s$.

In other words, after scaling the structure by a factor of $s$, both the frequency and the field profile are scaled by the same factor. So in designing photonic crystals, the wavelengths of certain features can be controlled by adjusting the lattice constants of the photonic crystals.

The origin of bandgap can be explained by the Variational theorem which states that a mode tends to concentrate its electric-field energy in high dielectric regions while remaining orthogonal to the modes below it in frequency ${ }^{1}$. In a photonic crystal band diagram the lower band is known as dielectric band where as the rest of the bands are known as air bands. In the air bands energy is concentrated in lowest dielectric regions to maintain orthogonality in the Variational theorem principle, thus creating a gap between the bands. There are no eigen value solutions for the master equation in this bandgap region as shown in Figure 2.1. A design rule of thumb is that the higher the 


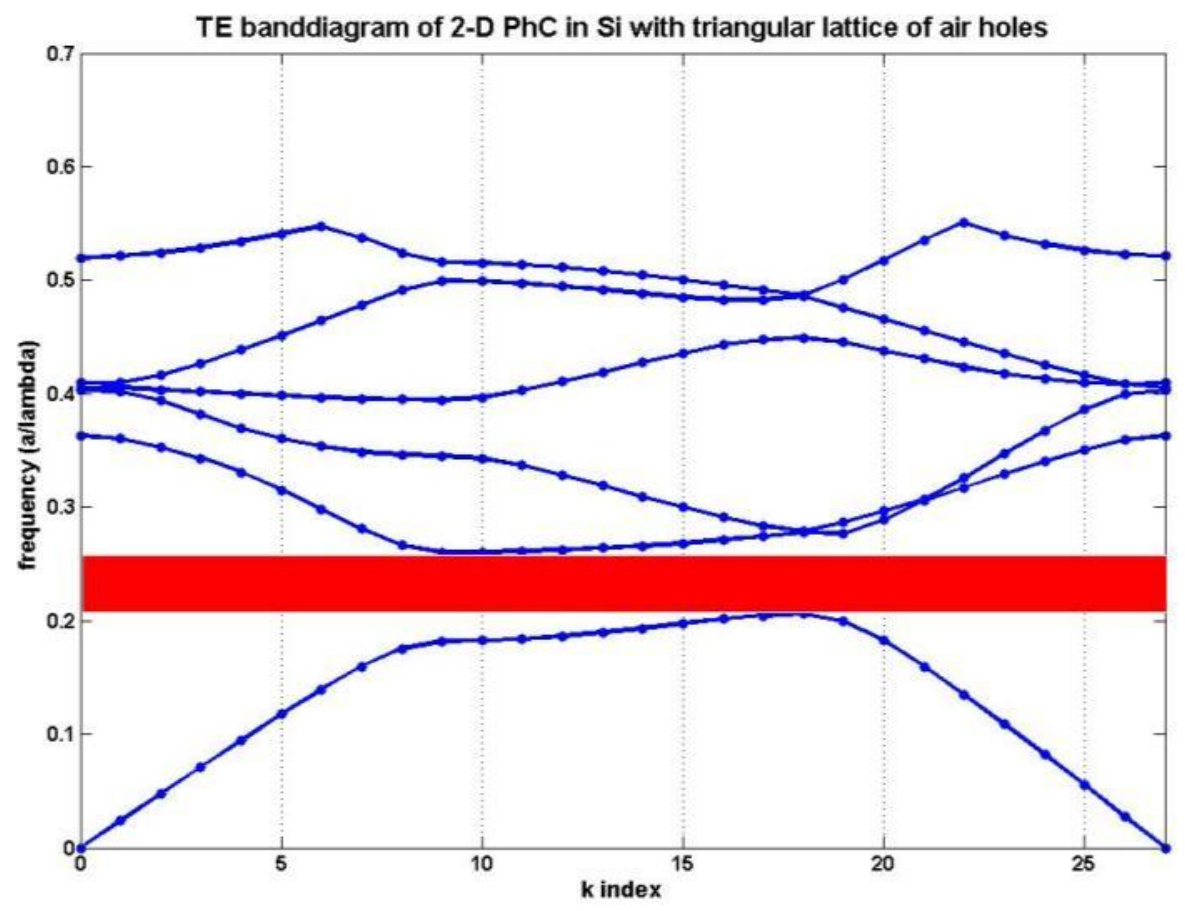

Figure 2.1 TE Band diagram of triangular lattice of air $(n=1)$ holes in $\mathrm{Si}(\mathrm{n}=3.45)$ with radius 0.28a has a gap-to-mid-gap ratio of $23 \%$

dielectric constant contrast of materials comprising the photonic crystal, the wider the bandgap. So, high dielectric contrast should be chosen whenever possible.

\subsection{1-D Photonic Crystal Theory}

One dimensional photonic crystals are the simple photonic crystal structures that consists of alternating layers of dielectric films as shown in Figure 2.2 . This kind of structure also known as Bragg mirror which acts as a mirror for specified range of frequencies. One dimensional photonic crystal can also localize modes if there are defects present in its structure. The working of a one-dimensional photonic crystal can be understood by considering a plane wave propagating through the material and accounting for the multiple reflections and transmissions that occur at each interface and the associated phase changes that occur for plane waves propagating from layer to layer. Fresnel's equations can be used to determine the amplitude and phase of the reflected and transmitted waves at an interface in terms of the refractive indices of the multilayer film. 


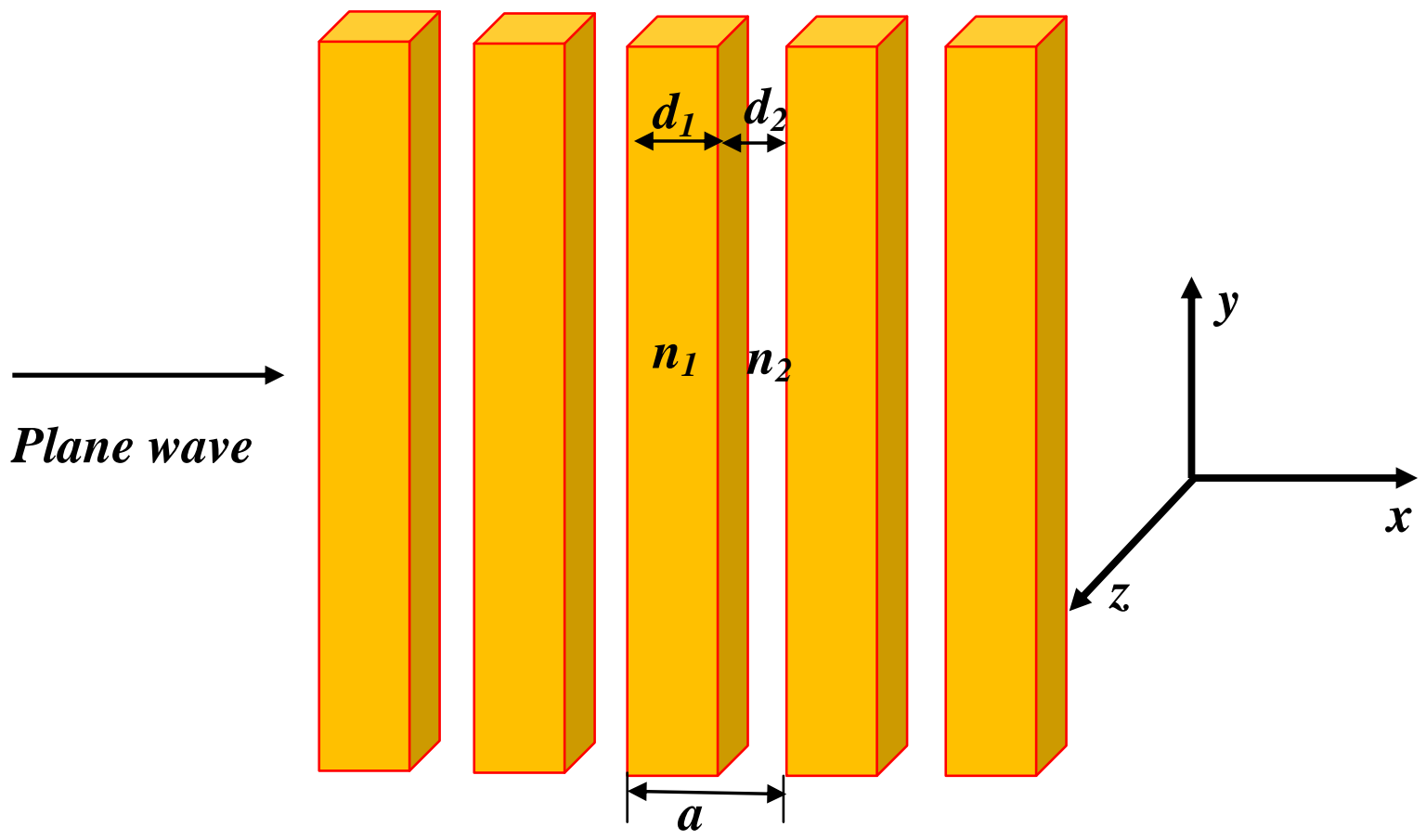

Fig 2.2 1-D Photonic Crystal ${ }^{1}$

In a 1-D photonic crystal, the photonic band gap depends on the difference in the dielectric constant or the thickness of dielectric material $\left(d / a=\left(d_{1}+d_{2}\right) / a\right)$. According to perturbation theory, if either the dielectric constant is weak $(\Delta \varepsilon / \varepsilon \ll 1)$ or the thickness is small then the bandgap can be expressed as

$$
\omega / \omega_{m} \approx \Delta \varepsilon / \varepsilon \cdot \operatorname{Sin}(\pi d / a) / \pi
$$

For two materials with refractive indices $n_{1}$ and $n_{2}$ and thickness $d_{1}$ and $d_{2}$ (where $a=d_{1}+d_{2}$ ) the normal incidence bandgap can be maximized when ${ }^{1}$

$$
d_{1} n_{1}=d_{2} n_{2}
$$

Upon substitution of, $d_{2}=a-d_{1}$ in Eqn 2.19:

and

$$
\begin{aligned}
& d_{1}=a n_{2} /\left(n_{1}+n_{2}\right) \\
& d_{2}=a n_{1} /\left(n_{1}+n_{2}\right) .
\end{aligned}
$$

In this case, the mid-gap frequency $\omega_{m}$ is given by

$$
\omega_{m}=\frac{n_{1}+n_{2}}{4 n_{1} n_{2}} \cdot \frac{2 \pi c}{a}
$$

Substitution of the vacuum wavelength $\lambda_{m}=2 \pi c / \omega_{m}$ satisfies the relations $\lambda_{m} / n_{1}=4 d_{1}$ and $\lambda_{m} / n_{2}=4 d_{2}$ which denote that each layer is a quarter wave length in thickness for maximum photonic bandgap. The gap-to- midgap ratio' between the first two bands of a quarter wave stack can be given as ${ }^{1}$ 


$$
\frac{\Delta \omega}{\omega_{m}}=\frac{4}{\pi} \sin ^{-1}\left(\frac{\left|n_{1}-n_{2}\right|}{n_{1}+n_{2}}\right)
$$

From the above relation it can be calculated that bandgap is a maximum only for a quarter wavelength stack of materials.

\subsection{Photonic Crystal Slab and Off-Axis Propagation}

All the above derived formulae are for photonic crystals with normal incidence light propagation, defined here as on-axis propagation. The one major difference between on-axis and off-axis propagation is that there are no bandgaps in the off-axis propagation. This is because in the off-axis directions there is no periodic regime to scatter light and setup the conditions for a bandgap to be present. Another difference is that off-axis propagation has no degenerate bands because of lack of rotational symmetry.

In Figure 2.3 it can be observed that the two polarizations are linear at longer wavelengths although they have different slopes. This long wavelength phenomenon is important in all photonic crystals irrespective of geometry. At longer wavelengths, the electromagnetic wave cannot differentiate the fine structures of a photonic crystal and it sees the structure as a homogenous medium with an effective dielectric constant. In this region of wavelengths (where $\lambda>>$ material period), the diffraction is negligible.

The effective dielectric constant of a two material composite of which one is air is given as ${ }^{1}$

$$
\varepsilon_{e f f}=\varepsilon_{\text {air }} f+(1-f) \cdot \varepsilon_{p h c}
$$

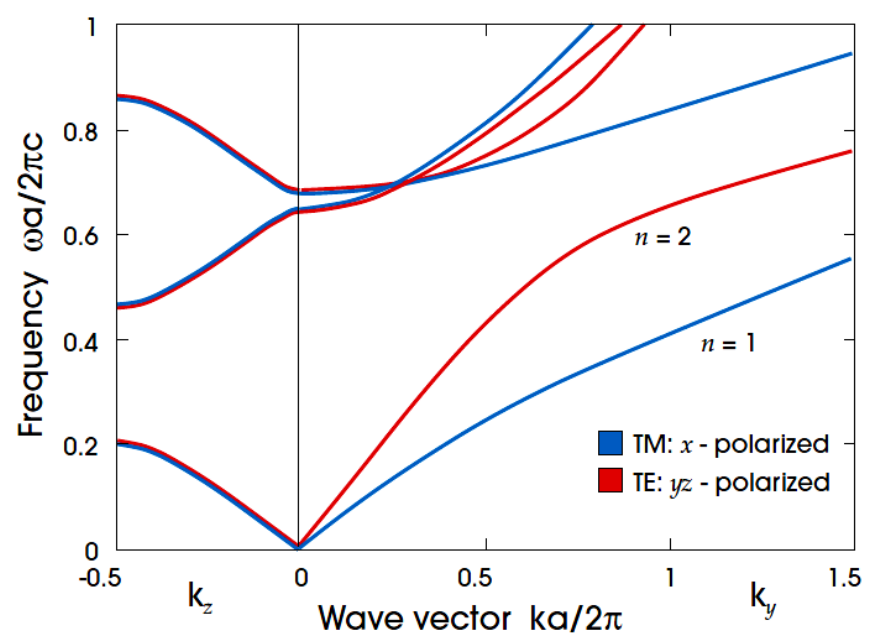

Fig 2.3 Band diagram of a 1-D Photonic crystal with off-axis propagation ${ }^{1}$

where $\varepsilon_{e f f}$ is the effective dielectric constant, $\varepsilon_{\text {air }}$ is the dielectric constant of air, $\varepsilon_{p h c}$ is the dielectric constant of the photonic crystal material and $f$ is the air filing factor or air-fraction. 
The general limits of an effective dielectric constant is given by ${ }^{1}$

$$
\left(f_{1} \varepsilon_{1}^{-1}+f_{2} \varepsilon_{2}^{-1}\right)^{-1} \leq \varepsilon_{e f f} \leq f_{1} \varepsilon_{1}+f_{2} \varepsilon_{2}
$$

where $\varepsilon_{1}, \varepsilon_{2}$ are the dielectric constants of the two materials and $f_{1}, f_{2}$ are the volume fractions of the materials.

At wavelength $\lambda$ close to the material period, properties associated with the structural periodicity which allows the dispersion curve mapping can be characterized ${ }^{33}$.

\subsection{Piezoelectric properties of Nitride Thin Films}

Aluminum- and Gallium-Nitride (AIN and GaN) thin films have high chemical and mechanical stability under harsh conditions as well as to their piezoelectric properties ${ }^{34}$. PhCs made on these films exhibit low loss in the visible spectral range and possess high indices of refraction, which is necessary to obtain a wide photonic bandgap and better light confinement within their defects.

\subsubsection{Piezoelectricity}

The piezoelectric effect was discovered by Pierre and Jacque Curie in $1880^{35}$. The piezoelectric effect occurs when the charge balance within the crystal lattice of a material is disturbed. When there is no applied stress on the material, the positive and negative charges are evenly distributed so the resultant net charge is zero potential difference. When the lattice is slightly changed due to the stress caused by an applied force, the charge imbalance creates a potential difference; often as high as several thousand volts however, the current is extremely small.

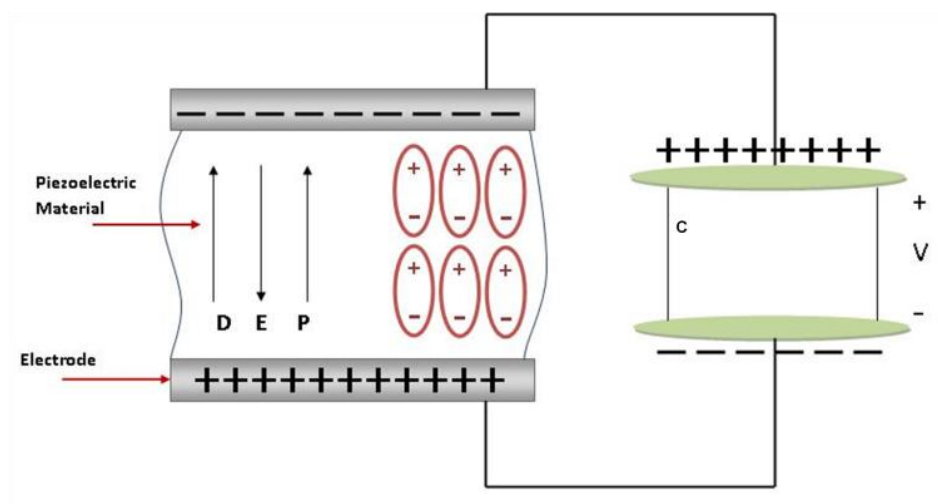

Figure 2.3 Schematic showing Piezoelectric Effect ${ }^{36}$

The converse piezoelectric effect occurs when an external electric field is applied to the piezoelectric element. In this case, the ions in each unit cell are displaced by electrostatic forces, resulting in mechanical deformation of the whole crystal as shown in Figure 2.3. 
If a voltage of the same polarity as the poling voltage is applied to a piezoelectric element, in the direction of the poling voltage, the element will lengthen and its diameter will become smaller. If a voltage of polarity opposite that of the poling voltage is applied, the element will become shorter and broader. If an alternating voltage is applied, the element will lengthen and shorten cyclically, at the frequency of the applied voltage.

\subsubsection{Piezoelectric constants}

A piezoelectric material or ceramic can be considered as a cluster of small individual piezoelectric crystals fitted in a random way. The behavior of a piezoelectric material can be explained by combining equations of its electrical behavior and elastic behavior (Hooke's law).

When an electric field is applied to a piezoelectric material, it produces a proportional strain in the material. It is expressed as $\boldsymbol{S}=\boldsymbol{d} . \boldsymbol{E}$ where $\boldsymbol{d}$ is the piezoelectric charge constant and $\boldsymbol{E}$ is the Electric field. The constitutive equations of a piezoelectric material derived from the above relations can be given as ${ }^{5}$

$$
\begin{gathered}
D=d T+\varepsilon^{T} E \\
S=s^{E} T+d E
\end{gathered}
$$

The tensor form of elastic compliance $(s)$ taking into account all symmetry relations ${ }^{37}$ can be expressed as

$$
\boldsymbol{s}=\left(\begin{array}{llllll}
\mathrm{s}_{11} & \mathrm{~s}_{12} & \mathrm{~s}_{13} & 0 & 0 & 0 \\
\mathrm{~s}_{12} & \mathrm{~s}_{11} & \mathrm{~s}_{13} & 0 & 0 & 0 \\
\mathrm{~s}_{13} & \mathrm{~s}_{13} & \mathrm{~s}_{33} & 0 & 0 & 0 \\
0 & 0 & 0 & \mathrm{~s}_{44} & 0 & 0 \\
0 & 0 & 0 & 0 & \mathrm{~s}_{44} & 0 \\
0 & 0 & 0 & 0 & 0 & 2\left(\mathrm{~s}_{11}-\mathrm{s}_{12}\right)
\end{array}\right)
$$

(i)Piezoelectric charge constant $(d)$ is the polarization generated per unit of mechanical stress $(T)$ applied to a piezoelectric material or, alternatively, is the mechanical strain $(S)$ experienced by a piezoelectric material per unit of electric field applied. It has the units of Coulombs/Newton in the direct piezoelectric effect and meters/volt in converse piezoelectric effect.

Its tensor form can be expressed as ${ }^{5}$

$$
\boldsymbol{d}=\left(\begin{array}{cccccc}
0 & 0 & 0 & 0 & \mathrm{~d}_{15} & 0 \\
0 & 0 & 0 & \mathrm{~d}_{15} & 0 & 0 \\
\mathrm{~d}_{31} & \mathrm{~d}_{31} & \mathrm{~d}_{33} & 0 & 0 & 0
\end{array}\right]
$$


(ii)Permittivity or dielectric constant $(\varepsilon)$ is the dielectric displacement per unit electric field.

(iii)Young's modulus $(Y)$ is an indicator of the stiffness (elasticity) of a ceramic material. $Y$ is determined from the value for the stress applied to the material divided by the value for the resulting strain in the same direction. Its units are Newton $/ \mathrm{m}^{2}$.

(iv)Mechanical Stress $(T)$ is the average amount of force exerted per unit area

(v)Elastic compliance $(s)$ is the strain developed in a piezoelectric material per unit of stress applied. $s D$ is the compliance under a constant electric displacement; $s E$ is the compliance under a constant electric field. Its units are $\mathrm{m}^{2} /$ Newton.

\subsection{Waveguide and Losses}

A waveguide is a structure which guides optical waves via total internal reflection. The light can be trapped in a waveguide when $n_{2}<n_{1}$ and $n_{3}<n_{1}$. For a guided mode to prevail in the waveguide the angle of incidence has to be greater than the critical angle $\left(\theta>\theta_{c}\right)$ where $\operatorname{Sin} \theta_{c}=n_{2} / n_{1}$ for lower boundary; $\operatorname{Sin} \theta_{c}=n_{3} / n_{1}$ for upper boundary.

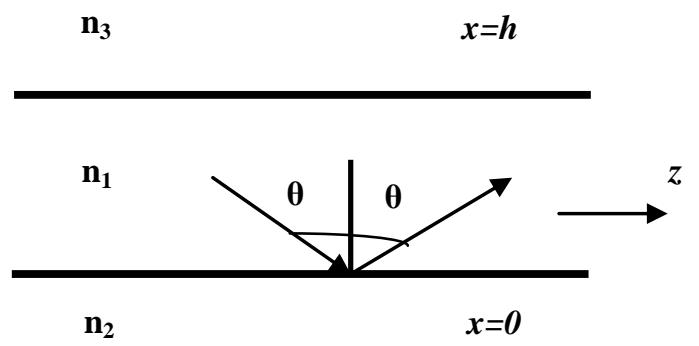

Figure 2. 4 Waveguide profile showing total internal reflection

(i) Symmetric slab dielectric Waveguide: A symmetric slab dielectric waveguide is a wave guide in which both sides of wave guiding region has same refractive index $n_{2}=n_{3}$

(ii) Asymmetric slab dielectric Waveguide: An asymmetric slab dielectric waveguide is a waveguide in which the region above $x=h$ is air and the region below $x=0$ is substrate $n_{2} \neq n_{3}$.

(iii) Mode condition: A mode is a spatial distribution of optical energy in one or more dimensions ${ }^{38}$. The two boundaries of the waveguide form a cavity. A cavity is resonant when round trip phase shift is $2 \pi \mathrm{m}$,

$$
\Delta \emptyset=\mathrm{m}(2 \pi) .
$$

Waves travelling at these allowed angles will interfere constructively, while the ones which are out of phase interfere destructively. Similar conditions exist to dictate sustainable modes in slab waveguide systems. A guided mode is a set of electromagnetic fields which maintain their transverse spatial distribution along the propagating 
direction. A guided wave consists of a non-planar wave decaying in $\pm x$ direction in the cladding region while maintaining a propagation constant of $k_{z}$ in the $z$ direction.

Waveguide Losses: The relative difference between the output power present at the ends of the waveguide to that of the power launched as an input gives the measure of attenuation. Coupling, scattering, absorption and radiation losses are important sources of attenuation in waveguides.

(a)Absorption Losses: Materials absorb light energy depending upon the operating wavelength and inherent material characteristics. If the absorption is in the visible spectrum, then is the material will be opaque. Light absorption occurring in waveguides due to the waveguide material is known as intrinsic absorption. Absorption due to impurities within the waveguide material is known as extrinsic absorption. Neglecting the loss from impurities, absorption loss is uniform and constant. These losses can be reduced by the choice of material and operating wavelength $^{39}$

(b)Scattering Losses $^{17}$ : These form the major part of all the losses and must be minimized for better operation of any waveguide device. There are many types of scattering losses based on their origin. There are two types of scattering losses based on the output frequency. They are Linear Scattering and Non-linear scattering losses.

(c)Radiation Losses or Bending Losses ${ }^{17}$ : Radiation losses occur at bends or curves in the waveguide path. These losses usually occur as the evanescent tails' velocity exceeds the velocity of light in the cladding and hence the light is radiated from the waveguide. During bends, the portion of the evanescent tail in the cladding region has to travel faster than the light in the core to stay with the wave, which is not possible, and hence, that part of light is lost in radiation modes. Radiation losses occur in straight waveguides when scattering events couple optical power contained in guided modes into radiation modes which then carry the power from the waveguide.

(d) Coupling Losses ${ }^{17}$ : Losses that occur during coupling of light to and from the waveguide are termed coupling losses. These can be reduced only by using efficient couplers and the losses would vary with the optical schemes employed to input light into the waveguides. These losses do not depend on any of the waveguide parameters but depend on the physical operation of the device. As an example, losses in prism coupling can be reduced by coupling the light at the coupling spot and, thus, enabling maximum amount of power transfer into the waveguides. In thin dielectric waveguides the major source of attenuation is through scattering. Hence all the losses are usually neglected while measuring losses in the waveguide. Assuming attenuation is present solely due to scattering, the intensity at a point in the propagation path of the waveguide can be approximated by, 


$$
I(z)=I_{o} \exp (1-\alpha z)
$$

where $I_{o}$ is the initial intensity at $z=0$. The loss (in $\mathrm{dB}$ ) is related to $\alpha$ by the following expression ${ }^{16}$

$$
\operatorname{Loss}(\mathrm{dB} / \mathrm{cm})=4.3 \alpha\left[\mathrm{cm}^{-1}\right] \text {. }
$$




\section{CHAPTER 3: THEORETICAL MODELING}

\subsection{Introduction}

A variety of modeling tools can be used to theoretically understand the optical and mechanical properties of thin film devices. In this Chapter, photonic crystal optical modeling using MIT Photonic Bands (MPB) and piezoelectric modeling of lattice structures using ANSYS are discussed. This discussion also includes waveguide modeling using Opti-BPM (Beam Propagation Method) software is also discussed for determining the optical loss properties of thin films.

\subsection{PhC and Structural Modeling Tools:}

MPB (MIT Photonic Bands) ${ }^{40}$ is modeling software for the calculation of the band structure (dispersion relations ${ }^{41}$ ) and electromagnetic modes for periodic structures such as photonic crystals, waveguides, and resonator systems e.t.c. MPB is a UNIX or LINUX based software which is freely available and it was developed on Scheme language ${ }^{40}$ which is very simple yet powerful. MPB does a direct computation of the eigen states and eigen values of Maxwell's equations using a plane-wave expansion technique ${ }^{42}$.Solutions to the master equation solved by MPB are scaled in terms of lattice parameter $a$. Therefore, all photonic band structures are plotted as normalized wavelength $\left(a / \lambda_{0}\right)$ vs. wave vector $k$. Hence we can determine solutions at any length scale.

ANSYS is a sophisticated and comprehensive finite element modeling tool that is available commercially. It can be used for numerically solving a wide variety of mechanical structural analyses ${ }^{43}$. However, it has capabilities in many different physics fields such as static structural, nonlinear, thermal, implicit and explicit dynamics, fluid flow, electro magnetics, and electric field analysis e.t.c. It can also perform multi-domain coupled field analyses combining one or more of these different simulation domains. Piezoelectric analysis is a form of coupled field analysis because it takes electric as well as elastic properties of the material into account.

\subsubsection{MPB and FEA of 2-D Photonic Crystal}

MPB was used to design 2-D photonic crystal device geometry in $\mathrm{GaN}^{6,7,8,44}$, which exhibits piezoelectric properties that could potentially be used as a tuning mechanism. The lattice geometry errors inherent to $\mathrm{PhC}$ fabrication could potentially cause a shift in bandgap which will, in turn, affect the intended functionality of the PhC device. In order to better understand the effect of geometrical parameter variation on the bandgap of a photonic crystal, efforts were made to analyze the coupled results from MPB optical and ANSYS structural modeling of PhCs. Even though GaN 
exhibits piezoelectric properties, it has very low piezoelectric coefficients which make it less useful in the direct tuning of geometrical parameters of PhCs. Because of this, AlN, which has higher piezoelectric coefficients than $\mathrm{GaN}$ and is another nitride material being studied by our group, was used for further exploring the effect of variations in lattice parameters on PhC bandgap. The band diagram of 2-D PhCs in AlN with a geometry derived from the $\mathrm{GaN} \mathrm{PhC}$ discussed in [5] and [6] is shown in Figure 3.1. Wave vector, $k$ with indication of the irreducible Brillouin zone points are plotted on the $\mathrm{x}$-axis and normalized frequency on the $\mathrm{y}$-axis. Because of the scalar invariance of Maxwell's equations, the gap-to-mid-gap ratio is a excellent way of articulating photonic bandgaps independent of the scale of the photonic crystal, where

$$
\% \text { bandgap }=\frac{\text { gap top wavelengt } h-\text { gap bottom wavelengt } h}{\text { gap mid }- \text { point }}
$$

TM banddiagram of 2-D PhC in AIN with triangular lattice of air holes

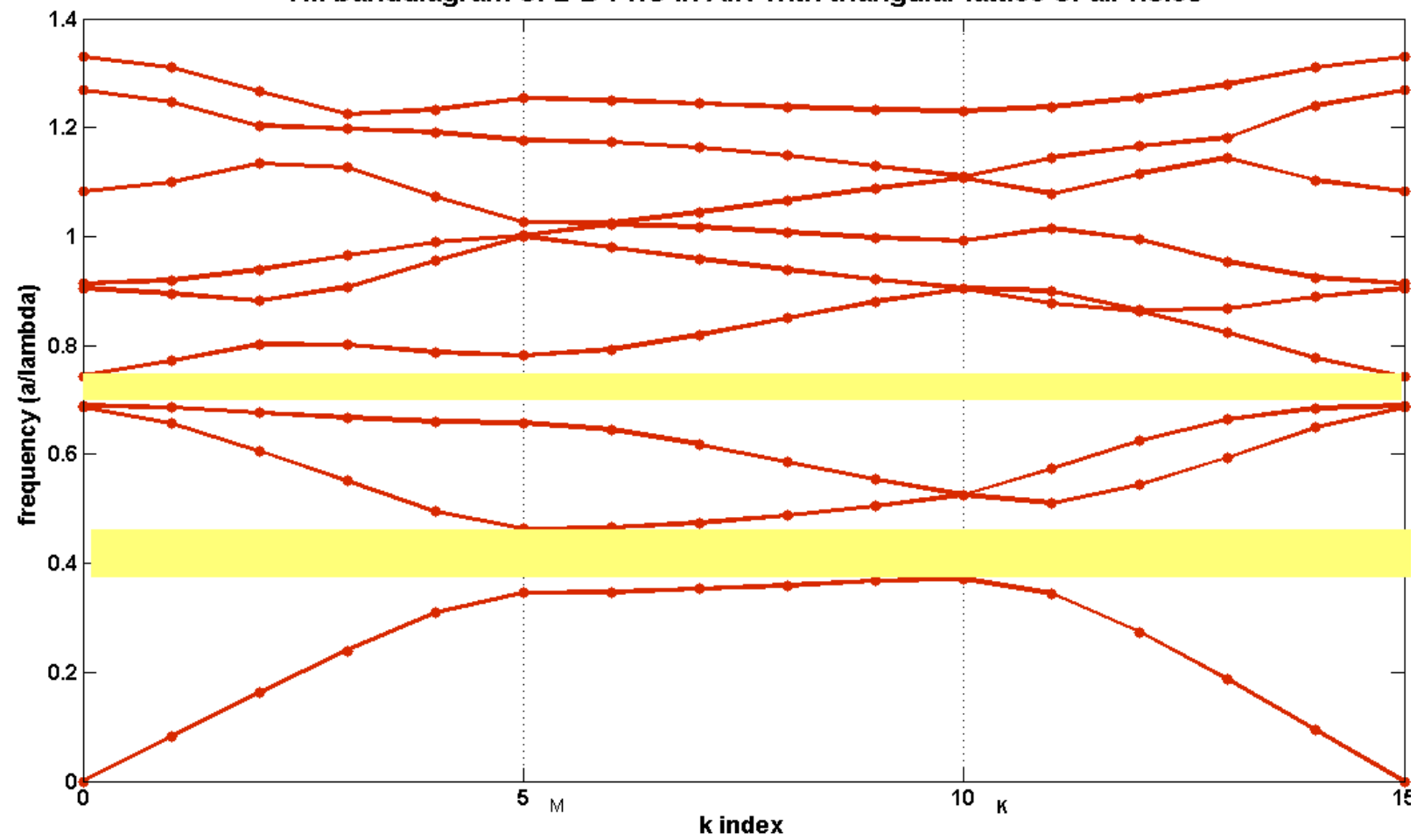

Figure 3.20 TM Band Diagram of 2-D PhC with triangular lattice of air holes in AIN with a radius of 0.29a has a gap-tomid gap ratio of $21 \%$ between $1^{\text {st }}$ and $2^{\text {nd }}$ bands and $7 \%$ between $3^{\text {rd }}$ and $4^{\text {th }}$ bands

In an ideal 2-D photonic crystal, the material is assumed to be infinitely thick, but in reality there is a finite thickness to the photonic crystal. Such structures are known as photonic crystal slabs. The optical properties of slab structures differ from those of the infinite thickness case as there is confinement of modes in the z-direction (thickness). Even though a third dimension is added to the photonic crystal, they cannot be treated as 3-D photonic crystals as there is no periodicity in the z-direction. The finite thickness of the PhC slab leads to major differences in the band structure 
compared to the ideal 2-D case. First, the modes do not decouple anymore into TE and TM polarization. For a symmetric photonic crystal slab ( i.e upper and lower cladding layers are similar) since the central axis of the $\mathrm{PhC}$ is parallel to the plane of incidence, the modes can be labeled as even and odd modes (denoted by the addition of a ' $z$ ', zeven or zodd). In the case of even (or odd) modes, the magnetic field is even (or odd) with respect to the plane of symmetry. Secondly, not all the modes existing in the PhC slab are guided. Most of them couple with modes of the claddings and are either guided in the cladding or scattered out of the slab. This continuum of radiation states is called the light cone and is limited by the light line in the cladding. Thus, radiation and guided modes are separated by light line defined as ${ }^{45}$

$$
\omega=c k / n_{\text {clad }}
$$

where $\omega$ is angular frequency, $k$ is the magnitude of wave vector, $c$ is the speed of light in vacuum and $n_{\text {clad }}$ is the refractive index of the cladding. The TE-like or zeven band diagram of 2-D PhC slab in AlN is shown in Figure 3.2.

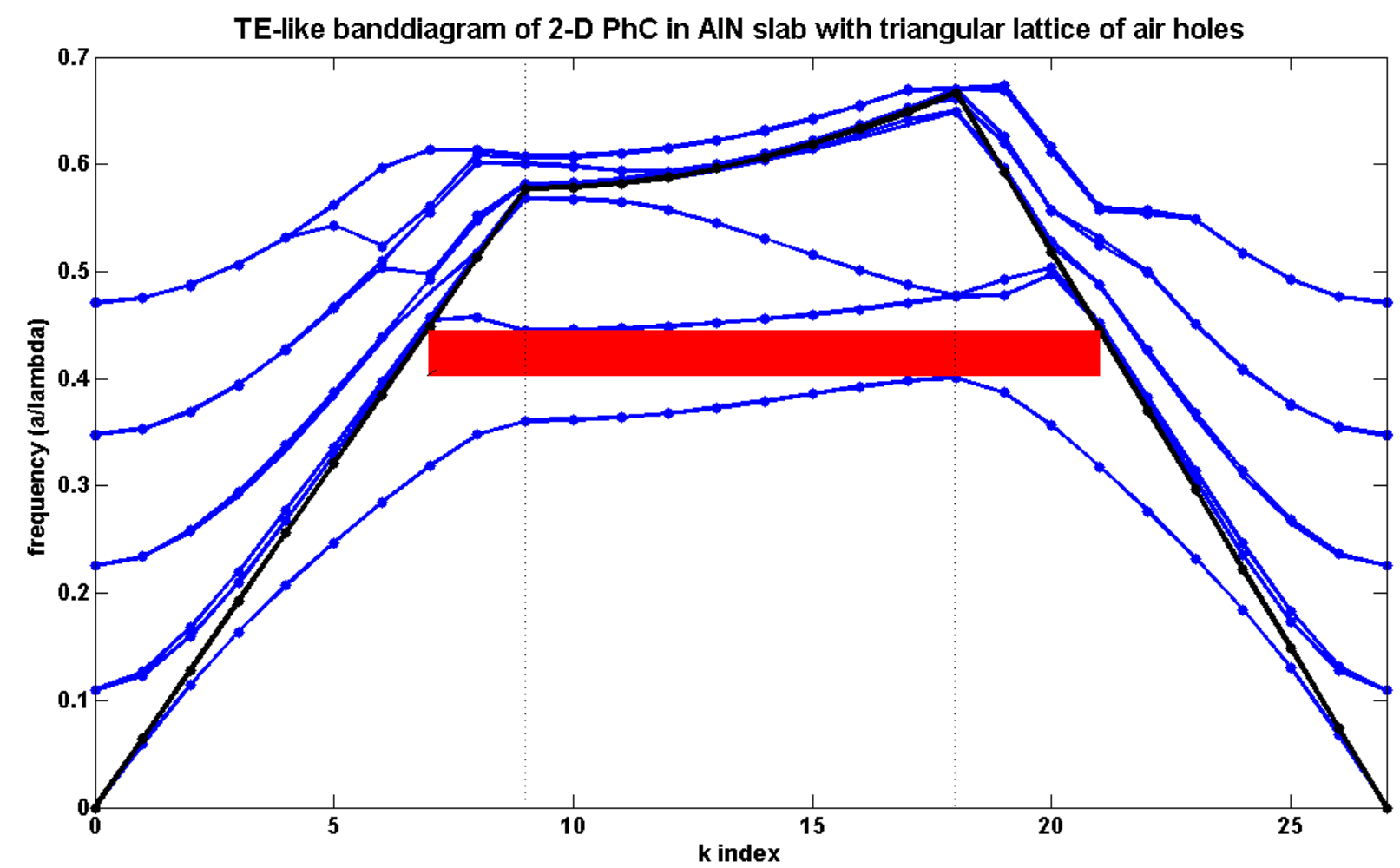

Figure 3.21 TE-like or zeven band diagram of 2-D PhC in AIN slab with a gap-to-midgap ratio of $10 \%$ between the first two guided bands under the light cone.

The modes that lie under the light cone are known as guided modes and the modes above the light cone are called the radiation modes. The bandgap exists in the region below the light cone which is also defined as the region in 
which there are no guided modes under the light cone. The TE-like band structure of a symmetrical 2-D PhC in AlN with circular air holes surrounded by air cladding is shown in Figure 3.2. The slab thickness plays a vital role in the existence of bandgap in a photonic crystal slab. If the slab is too thick, then higher order modes are created which will lie slightly above the lowest order mode, pulling down the air band causing miniscule gap. If the slab is too thin, then the slab will provide only a weak perturbation on the background dielectric constant which produces weakly guided modes. The optimal slab thickness can be calculated as

$$
\text { slab thickness } h \sim \frac{1}{2 \omega_{\text {gap }- \text { bottom }} \sqrt{\varepsilon}}
$$

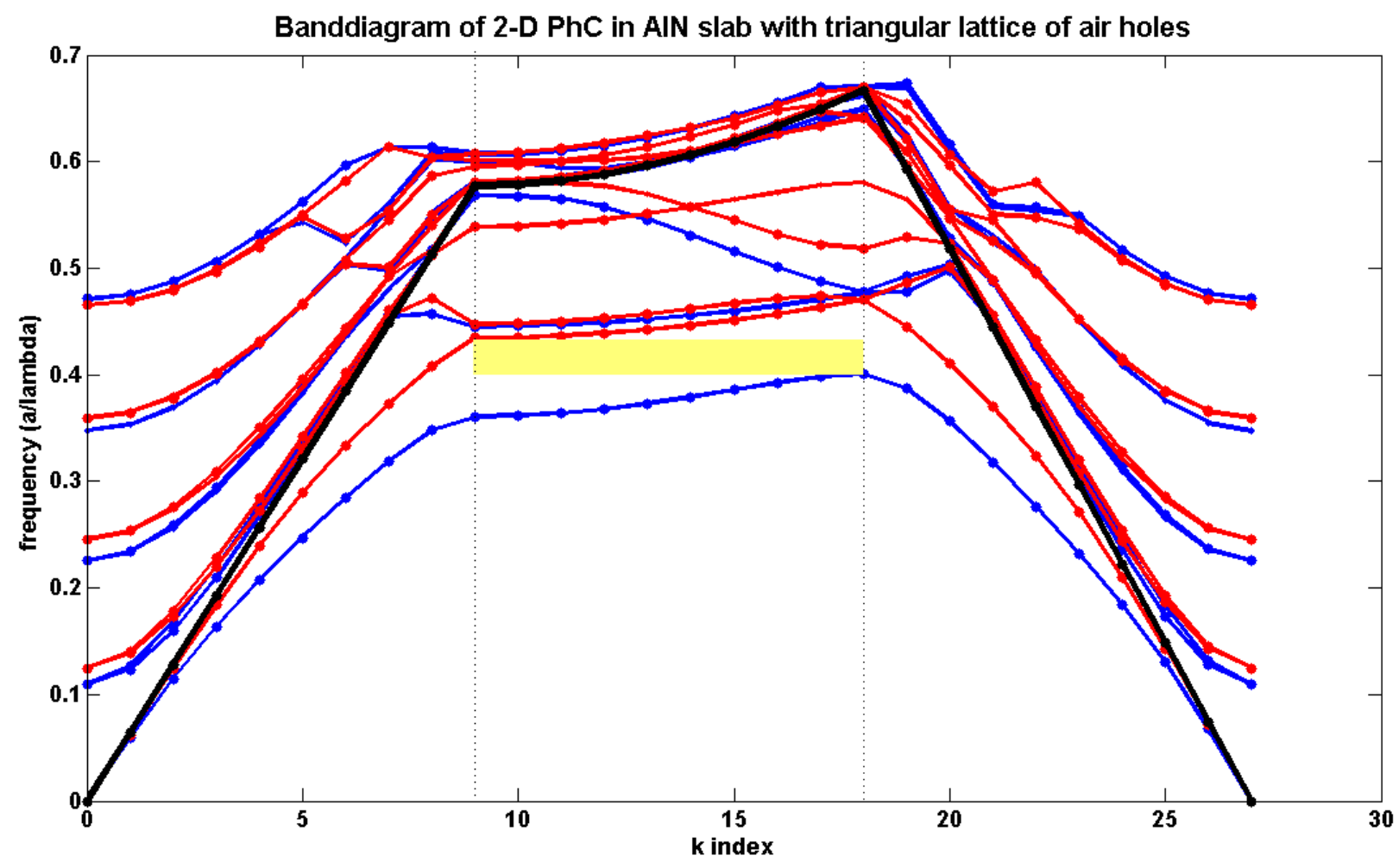

Figure 3.22 Band Diagram of 2-D Phc in AIN slab with a common band gap of $\sim 9.8 \%$ between the guided modes of TElike and TM-like bands

After determining device geometries which give a maximum bandgap to work at a specified wavelength via optical modeling, the structure is then transferred to ANSYS finite element analysis (FEA) software to calculate structural deformations and deflections using the embedded AC and DC voltage source tool within ANSYS.

The band structure for an asymmetrical photonic crystal slab (e.g. substrate and air are the two cladding layers) differs from that of symmetrical systems. In this case, vertical symmetry is broken and so the modes become inseparable and hence cannot be referred to as even or odd modes. Bandgap formation in such situation would be difficult if the substrate and the PhC material are not chosen properly ${ }^{9}$. 
The band diagram of a 2-D AlN PhC slab is shown in Figure 3.3. TE-like and TM-like bands are overlapped in this diagram to find the common band gap of the structure. The gap-to-midgap ratio of the portion where there is absence of guided modes was found to be $\sim 9.8 \%$ as shown the Figure 3.3 .

In general, the dielectric periodicity of 2-D photonic crystal is infinitely extended in two dimensions (in the plane of the slab). In ANSYS, the structure to be simulated divided into small, finite elements and further into nodes. Due to the limitation on the number of elements (or nodes) that can be used to divide the sample in ANSYS, larger structures such as infinite $\mathrm{PhC}$ patterns on a slab are difficult to analyze, because if a large structure is used with a smaller number of elements, the simulation resolution will be too large, resulting in erroneous results. Hence any structure should be finely divided into elements and then to nodes. Due to this limitation usage of large-area photonic crystals is not possible in our version of ANSYS. To prepare for this, simulations were done to see the effect of increasing the size of structure on deformation. The dimensions of the slab and the 2-D PhC pattern as derived in optical modeling were used in the FEA as shown in Table $3.1^{5,8}$.

Tale 3. 1 Lattice parameters of 2-D PhC used in FEA

\begin{tabular}{|l|l|}
\hline $\begin{array}{l}\text { Lattice } \\
\text { Parameters }\end{array}$ & Values(nm) \\
\hline Radius & 64 \\
\hline Lattice constant & 220 \\
\hline Thickness & 250 \\
& \\
\hline
\end{tabular}


Different lengths and breadths of $\mathrm{PhC}$ slab sizes were considered in the simulation. In order for ANSYS to run piezoelectric coupled field analysis, a dielectric matrix, a piezoelectric matrix, [e], and either a compliance matrix, [d], or stiffness matrix, [c], are required.

Tale 3. 2 Material Properties of AIN

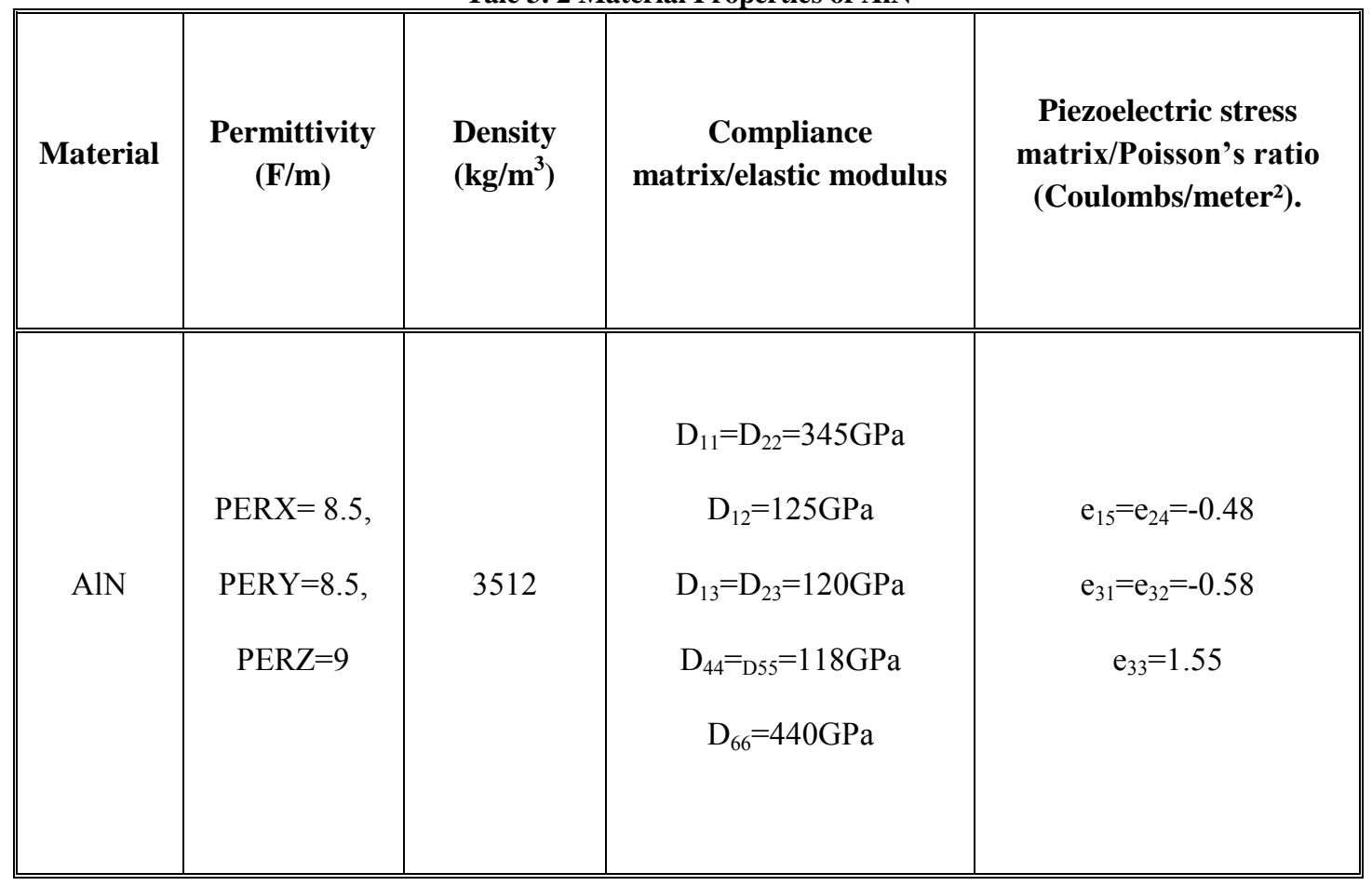

The dielectric property defines the electrical permittivity in Farads per meter. The piezoelectric matrix, [e], relates the electric field to stress, and typically has units of Coulombs per meter ${ }^{2}$. While ANSYS accepts stiffness data in terms of the elastic modulus and Poisson's ratio, it is more common in practice to define either the compliance matrix, [d], or the stiffness matrix, [c], for a piezoelectric coupled-field analysis. Material properties of AlN that were used in ANSYS are given in Table $3.2^{34}$.

Figure 3.4 is the visual representation of the mechanical deformation of a finite photonic crystal slab with lateral dimensions of $1 \mu \mathrm{m}$. In this case, the nodes on the corners of the slab are clamped which means that the nodes on the corners are defined as fixed points or given a zero displacement and a positive voltage of $20 \mathrm{~V}$ is applied on the upper portion of the slab and $0 \mathrm{~V}$ on the lower portion of slab. 


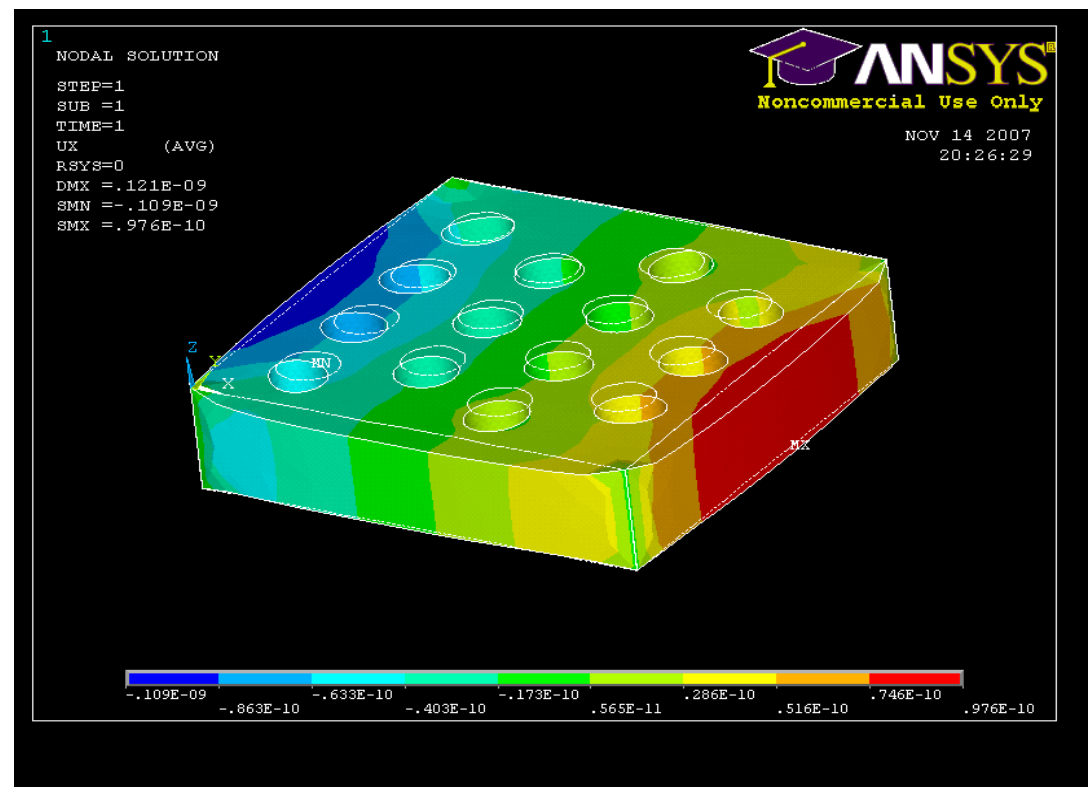

Figure 3.23 FEA of 2-D PhC showing the displacement in $\mathrm{x}$-direction when the edges of the slab are clamped

The variation of the dimensions of the slab vs. deformation in $-x,-y$ and $-z$ directions is shown in Figure 3.5.

Graph indicates that when the corners of the PhC slab are clamped then there is a high deformation in the lateral directions compared to the z-direction. Results indicate that deformation increases with the amount of piezoelectric material.

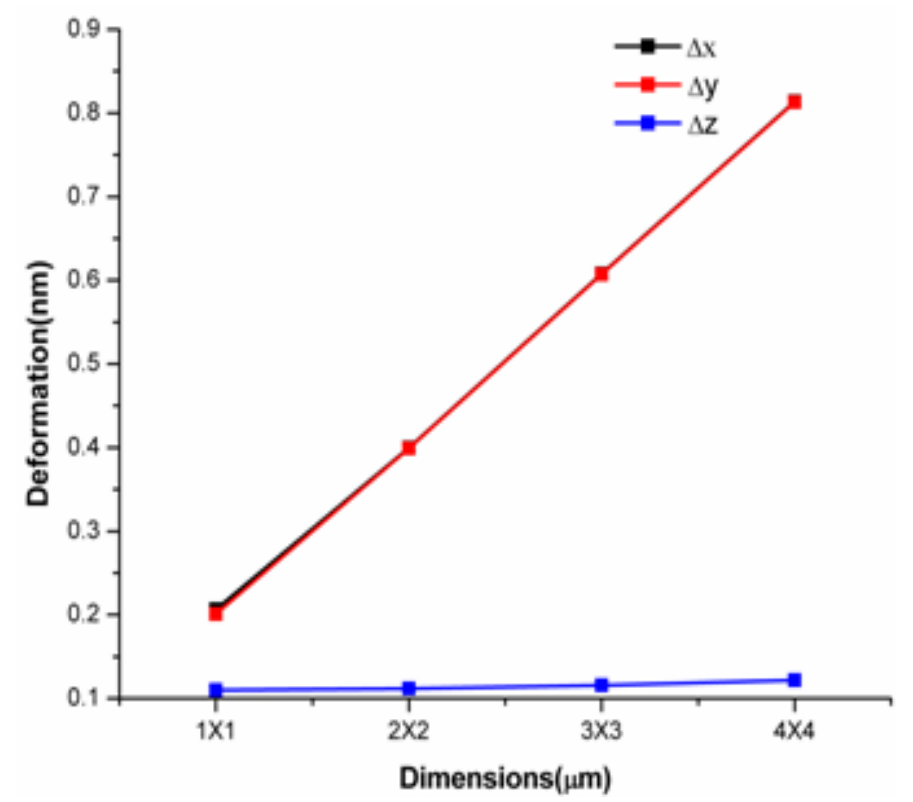

Figure 3.24 Dimensions of the slab vs. the deformation in $-x,-y$ and $-z$ directions

Variation of the level of deformation with changes in applied voltage is shown in Figure 3.6. These results indicate that, as expected, the mechanical deformation increase with applied voltage. Also, the level of deformation is high in 
the $-\mathrm{x}$, and $-\mathrm{y}$ directions compared to $-\mathrm{z}$ direction. It should be noted that ANSYS does not predict the effects of breakdown voltage in this type of simulation.

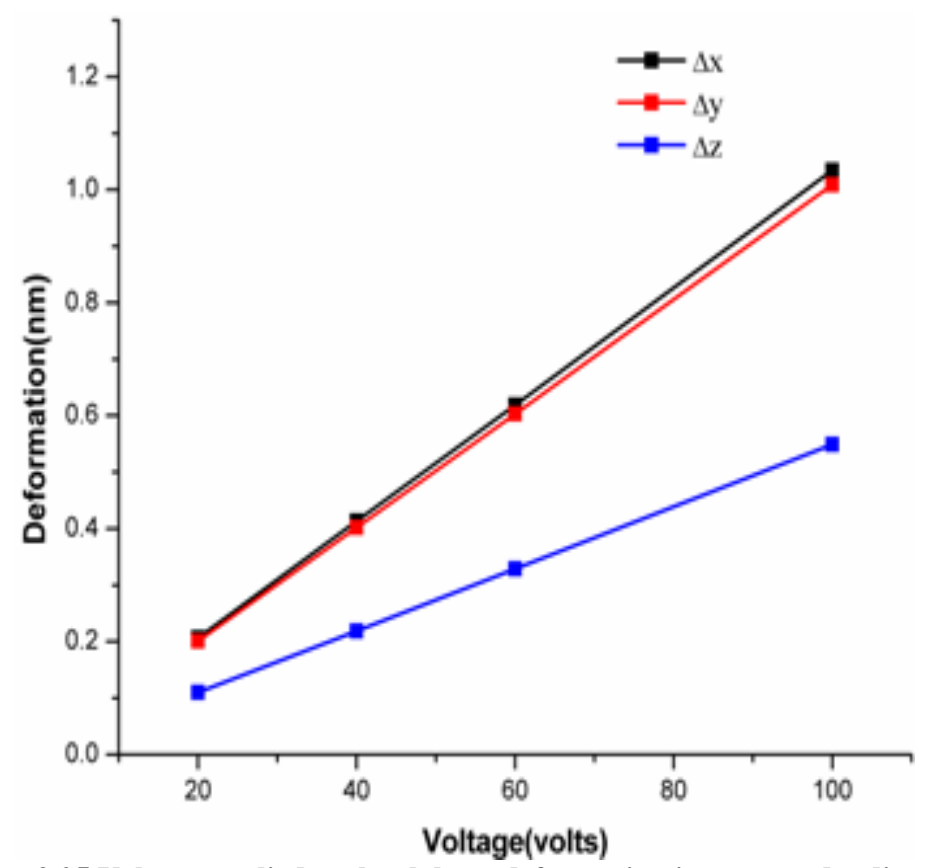

Figure 3.25 Voltage applied to the slab vs. deformation in $-x,-y$ and-z direction

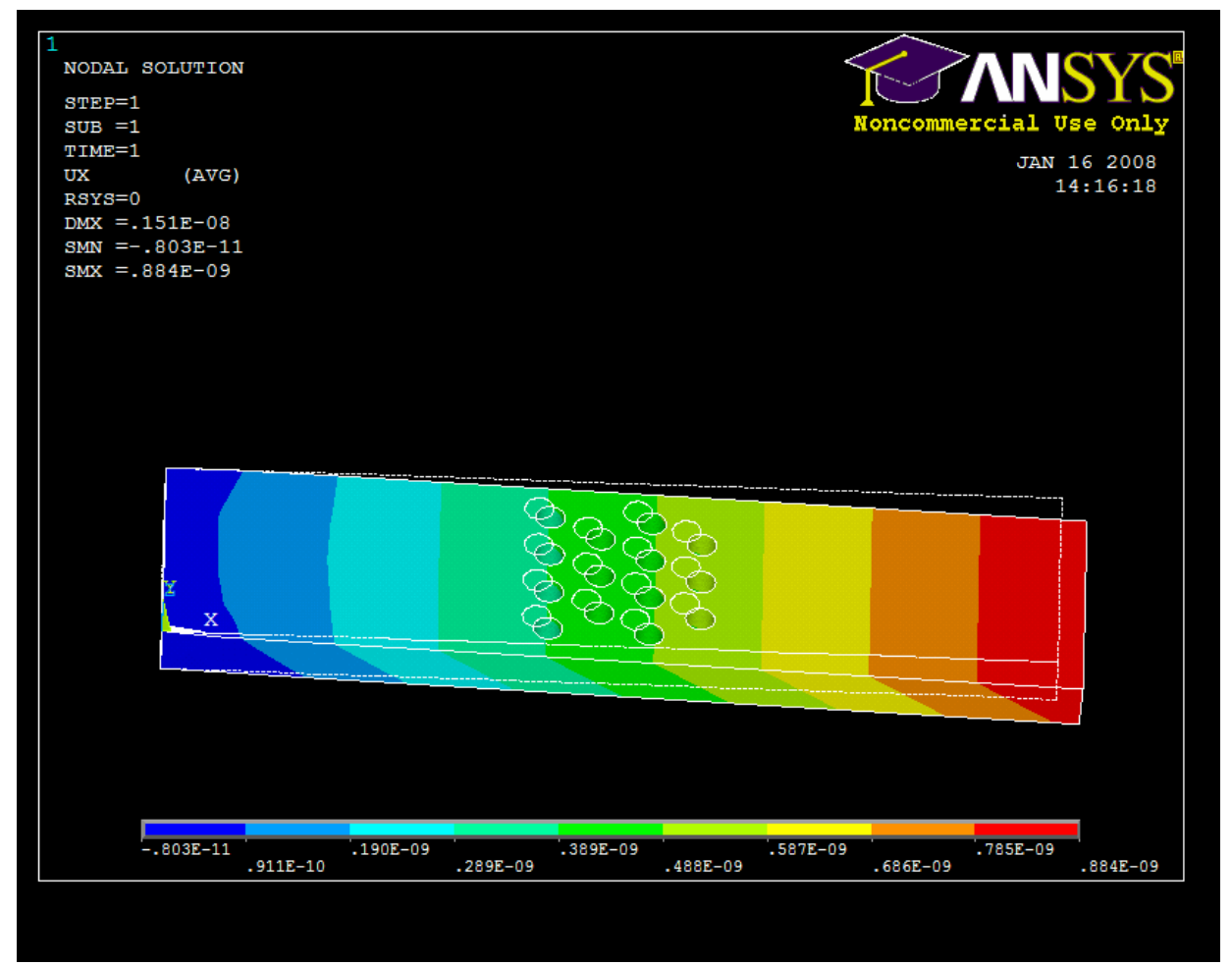

Figure 3.26 Deformation of 2-D PhC in -x direction when one side of the slab is clamped 
Another simulation was performed to observe how the photonic crystal behaves when clamped at different parts of the slab. The visual representation is shown in Figure 3.7. The slab used has dimensions of $4 \mathrm{X} 1 \mu \mathrm{m}$ with a triangular lattice of holes in the center $1 \mathrm{x} 1 \mu \mathrm{m}$ area. This result indicates that the degrees of freedom of the simulation greatly influence the piezoelectric deformation. In reality, if a piezoelectric slab is clamped to the substrate, the piezoelectric deformation observed in the z-direction will be less than the displacement of a suspended slab. This behavior was observed in the simulations.

DC Analysis of nanometer patterns of circular air holes on AIN (as shown in Figure 3.5 and 3.6) indicated subpicometer deformation of the air-hole lattice. This is considered to be insignificant in two ways. First, it is hard to measure such miniscule variations as the error in fabrication using E-beam Lithography is considered to be more than the geometrical variation due to piezoelectric effects. Second, the equipment required to measure such small scale deformations is not available in the lab. However, these modeling results indicate that the out-of-plane deformation in the overall slab surface is $\sim 10-20$ times larger than the deformation in the holes. This slab bending behavior can be used for tuning of bandgap by effectively changing the angle of incidence for a 1-D PhC. Therefore, 1-D PhCs were used for further exploring the geometrical variation effect on band gap as 1-D lattices will experience more deformation due to more material freedom compared to 2-D PhCs.

\subsubsection{1-D PhC Modeling}

\subsubsection{1-D PhC MPB Modeling}

Based on advantages discussed above, the effect of geometrical variations on bandgap was tested on 1-D PhCs. MPB was used to calculate the band diagram of 1-D PhCs in AlN. For an ideal 1-D PhC quarter wave stack as discussed in Chapter 2 condition gives the maximum bandgap.

Figures ( $3.8 \mathrm{a} \& \mathrm{~b}$ ) e demonstrate the TE and TM band diagrams of AIN 1D photonic crystal structure designed with the parameters of a quarter wave stack,

$$
\begin{aligned}
& \text { Width }_{\text {AlN }}=\frac{n_{\text {air }}}{n_{\text {air }}+n_{A l N}} \\
& \text { Width }_{\text {air }}=\frac{n_{\text {AlN }}}{n_{\text {air }}+n_{\text {AlN }}}
\end{aligned}
$$


TE Band diagram of AIN 1-D Photonic crystal

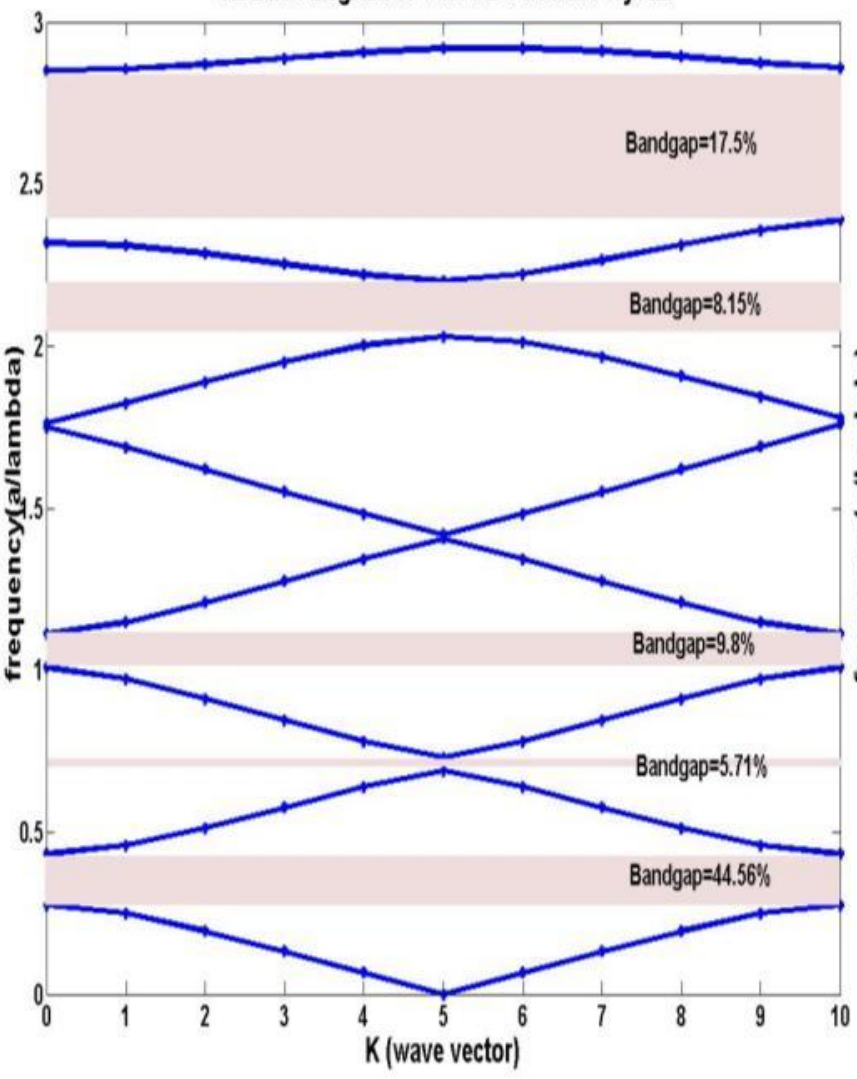

TM Band diagram of AIN 1-D Photonic crystal

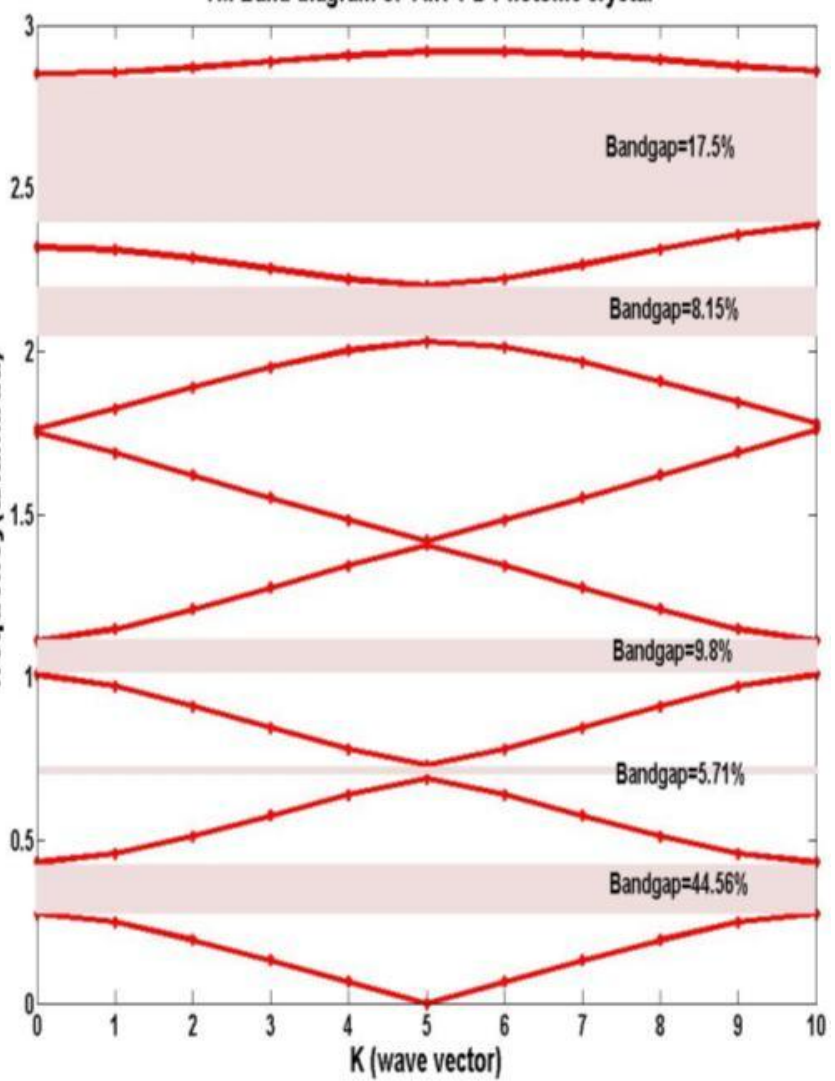

Figure 3. 27 (a\&b ) TE and TM Band diagram of 1-D PhC in AIN

where $W_{i d t h}$ air is the width of air grating, Width ${ }_{\text {AlN }}$ is width of the AlN grating. These are calculated with refractive indices $n_{a i r}=1$ and $n_{a l n}=2.11$. The widths are normalized with respect to the lattice constant in MPB. The maximum bandgap of a 1D PhC with an air-in-AIN lattice for TE and TM is found to be $44.56 \%$ ideally when the grating is assumed to be infinitely long in y and z-directions.

In general, the bandgap of a PhC increases with a larger refractive index contrast. Figure 3.9 demonstrates band gap change with variation in the width of the air grating in an ideal case. It is clearly noted that a $1 \mathrm{D}$ PhC gives maximum bandgap with the dimensions of quarter wave stack (location of vertical line in plot). From the plot, it can be observed that small variation in the width varies the optical bandgap by as much as $2 \%$ to $3 \%$. The non- linearity in the graph is due to the complex behavior of light interactions with the lattice parameters, which may cause higher order modes to shift down and reduce the bandgap. However, in reality, the length and thickness of the slab cannot extend to infinity: i.e. they have a finite length and thickness. To account for this characteristic, a second simulation was performed that accounts for the thickness of the PhC slab. Results are shown in Figure 3.10. 


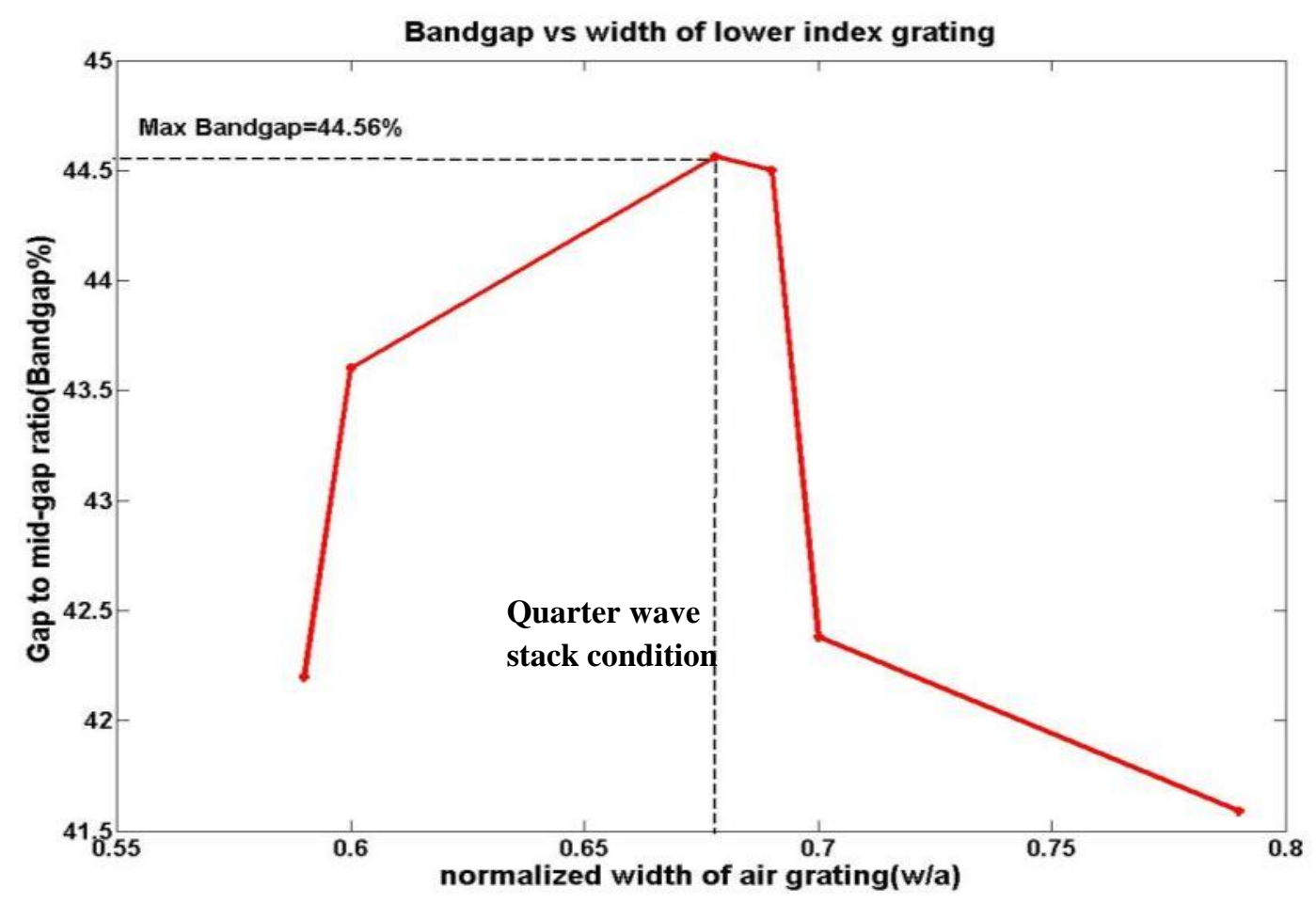

Figure 3. 28 Width of the air grating vs. gap-to-midgap ratio of 1-D PhC in AlN

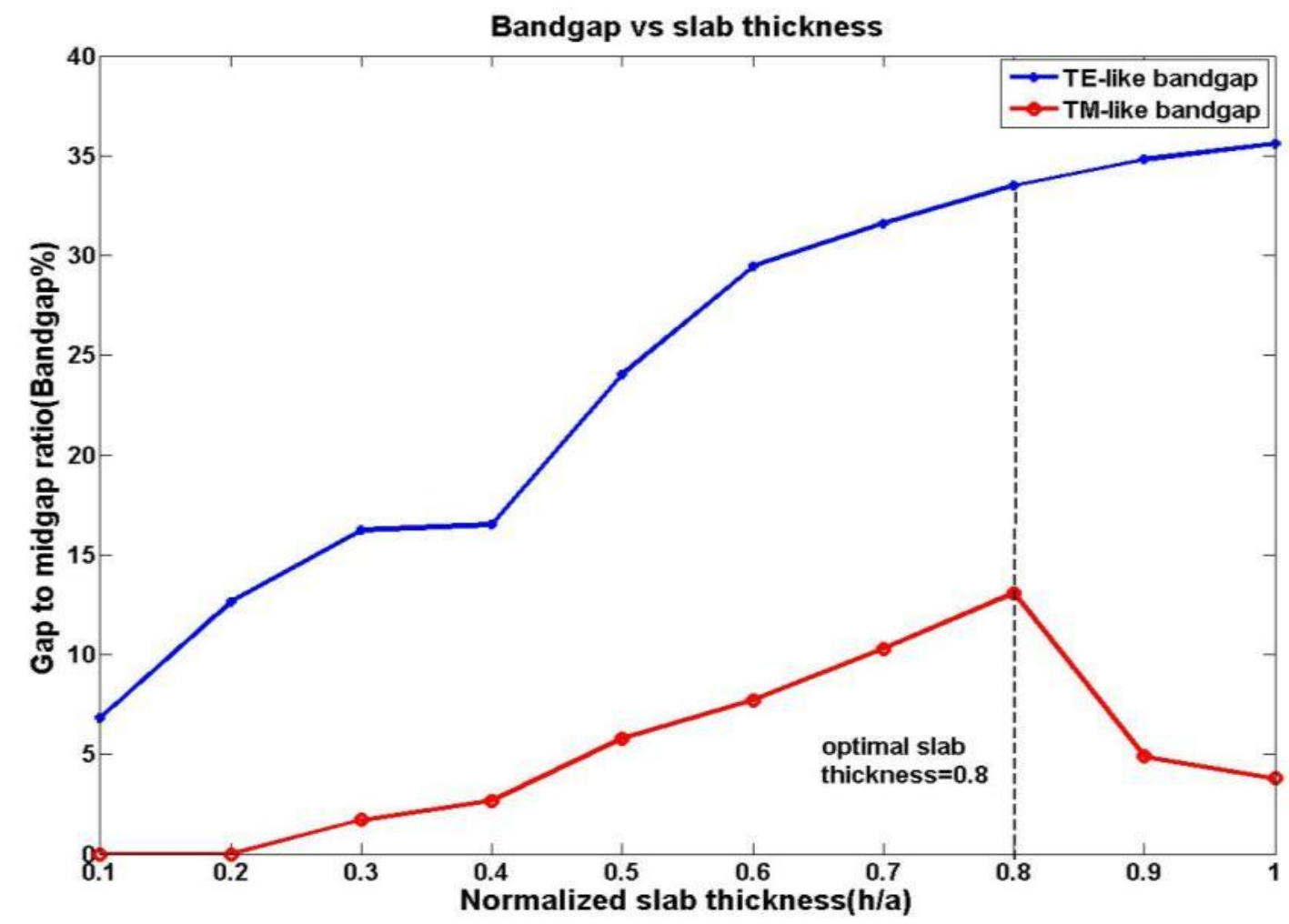

Figure 3.29 Slab thickness of 1-D PhC in AIN bounded by air cladding vs. gap-to midgap ratio 
Variation in the TE (blue) photonic bandgap is greater than the variation in the TM (red) bandgap. The higher order modes that lie slightly above lowest order mode reduce the optical bandgap as thickness is increased beyond an optimal value (using Eqn 3.3) which gives maximum bandgap. This is the main reason for the non-linearity in the graph. An optimum value of slab thickness is used to obtain the $\mathrm{PhC}$ design parameters based on normalized dimensions obtained from the simulation and desired operating wavelength in which our sensor architecture works. The lattice dimensions necessary for an optical bandgap which blocks a wavelength of $1.55 \mu \mathrm{m}$ which will be used for optical characterization are given in Table 3.3.

Tale 3. 3 Lattice parameters of 1-D PhC

\begin{tabular}{||c||c||}
\hline \hline$\lambda$ & $1.55 \mu \mathrm{m}$ \\
\hline Width $_{\text {AlN }}$ & $216.4 \mathrm{~nm}$ \\
\hline Width $_{\text {air }}$ & $456.6 \mathrm{~nm}$ \\
\hline $\boldsymbol{a}$ & $673 \mathrm{~nm}$ \\
\hline $\boldsymbol{h}$ & $538 \mathrm{~nm}$ \\
\hline
\end{tabular}

\subsubsection{ANSYS Modeling}

\subsubsection{2(a) DC Analysis and Harmonic Analysis of 1-D PhC:}

In the suspended slab design shown in Figure 3.11, the solid lattice structures are anchored on both ends by a larger area of unpatterned material. As maximum deflection is critical to obtain maxim um tunability, different models were considered for simulation. In a model without conductive electrodes, which are necessary for providing the potential necessary for piezoelectric deformation in actual fabricated devices, the variation in the width and height of 1D PhC are very small: on the order of picometers, as shown in Figure 3.12 


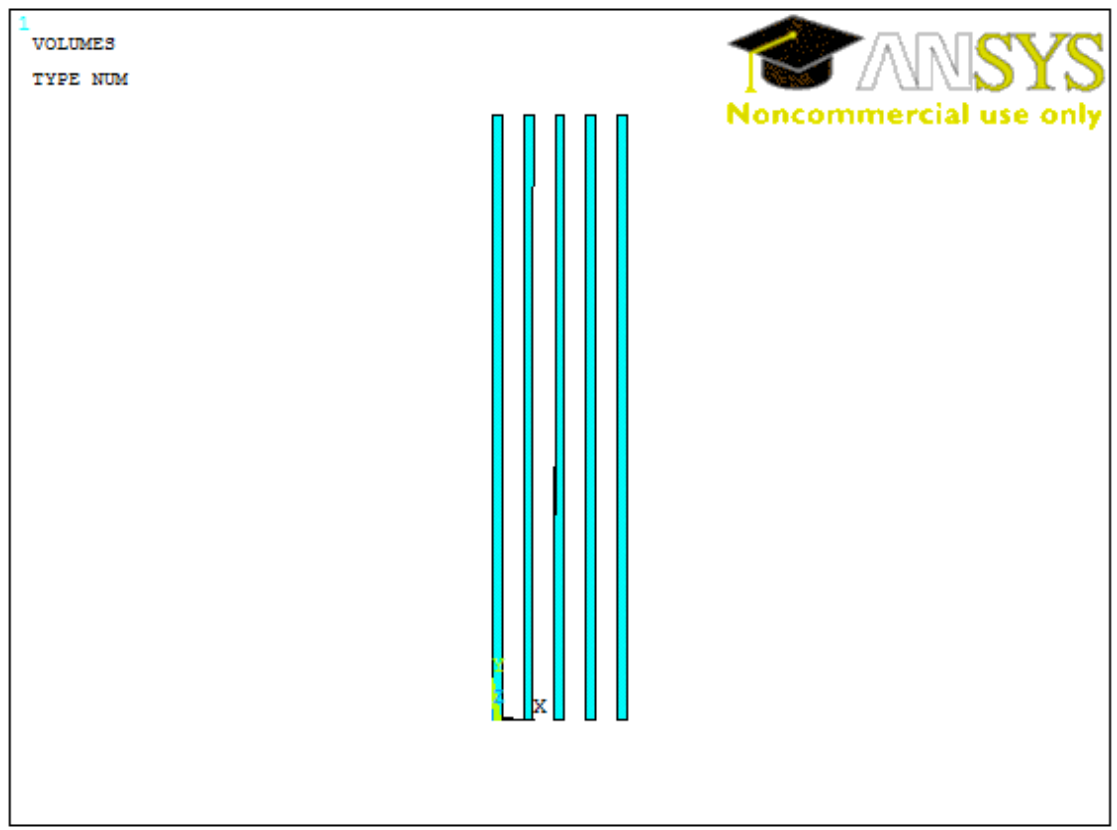

Figure 3. 30 Design of 1-D PhC in AIN

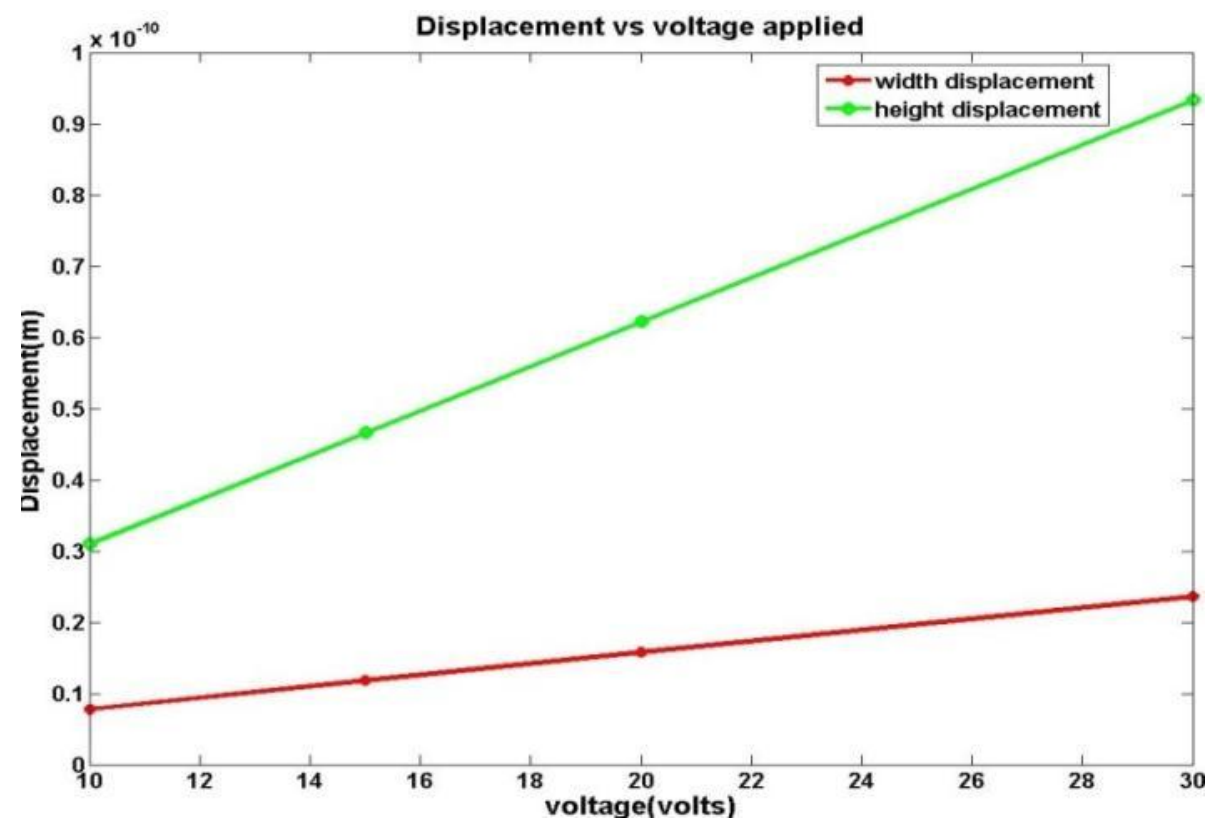

Figure 3. 31 Deformation vs. voltage for a model which gives minimum deflection

Not only will these changes in lattice parameters ( $h$ and $a$ ) be difficult to measure with certainty using experimental methods, but they are also too small to cause a significance shift in the photonic bandgap of the structure. 


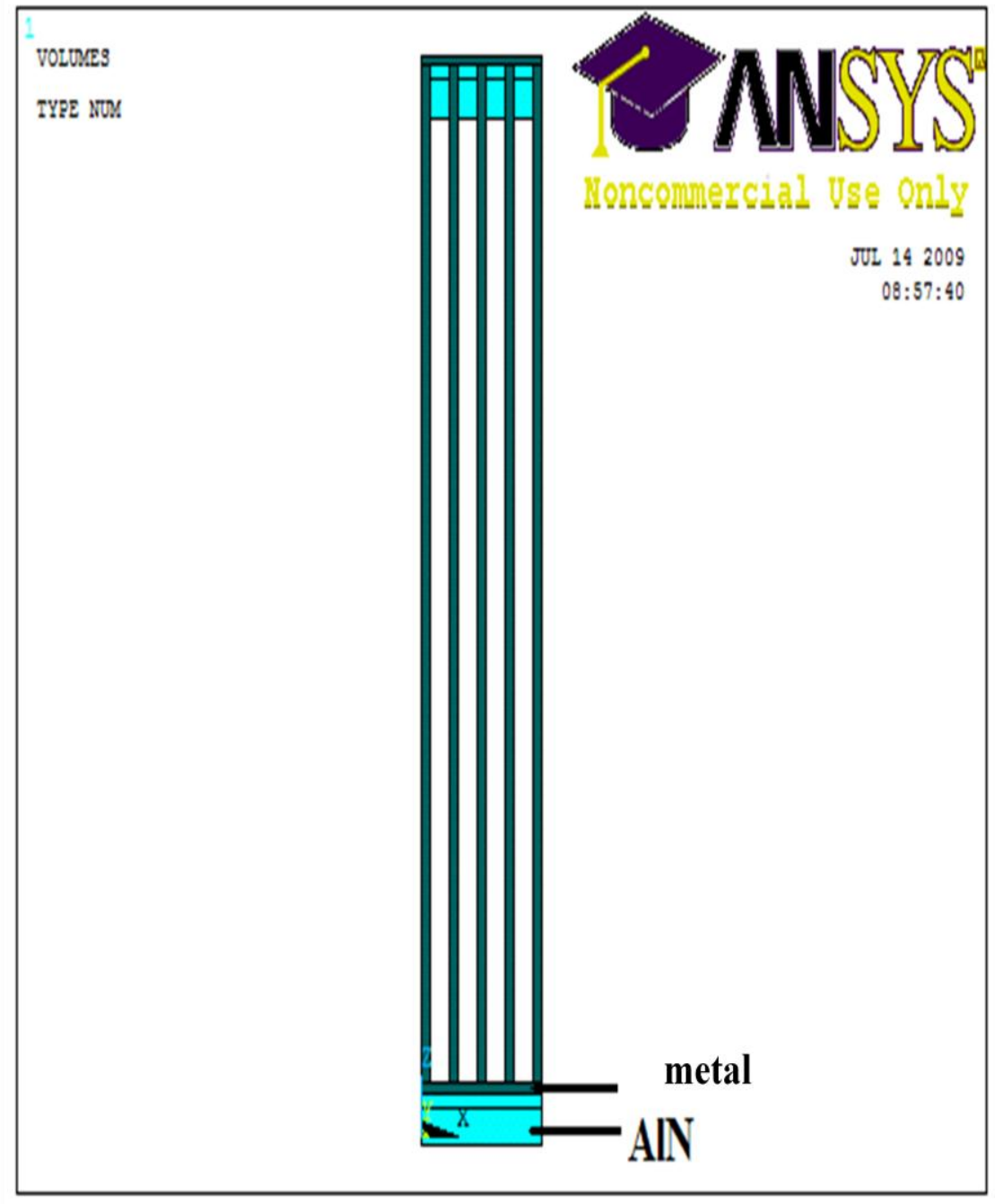

Figure 3. 32 Top-view of design of 1-D PhC with metal contact

In practice, conductive electrodes will be used to deliver the excitation voltage to the AlN $\mathrm{PhC}$ as shown in Figure 3.13. The addition of conductive electrodes not only offers a more accurate behavioral model, but also offers an avenue with which to cause higher degrees of mechanical deformation. Because of the difference in the rates of expansion of $\mathrm{PhC}$ and electrode materials with application of voltage, the beam exhibits a bow bending behavior. The amount of deflection varies with change in the electrode material. 


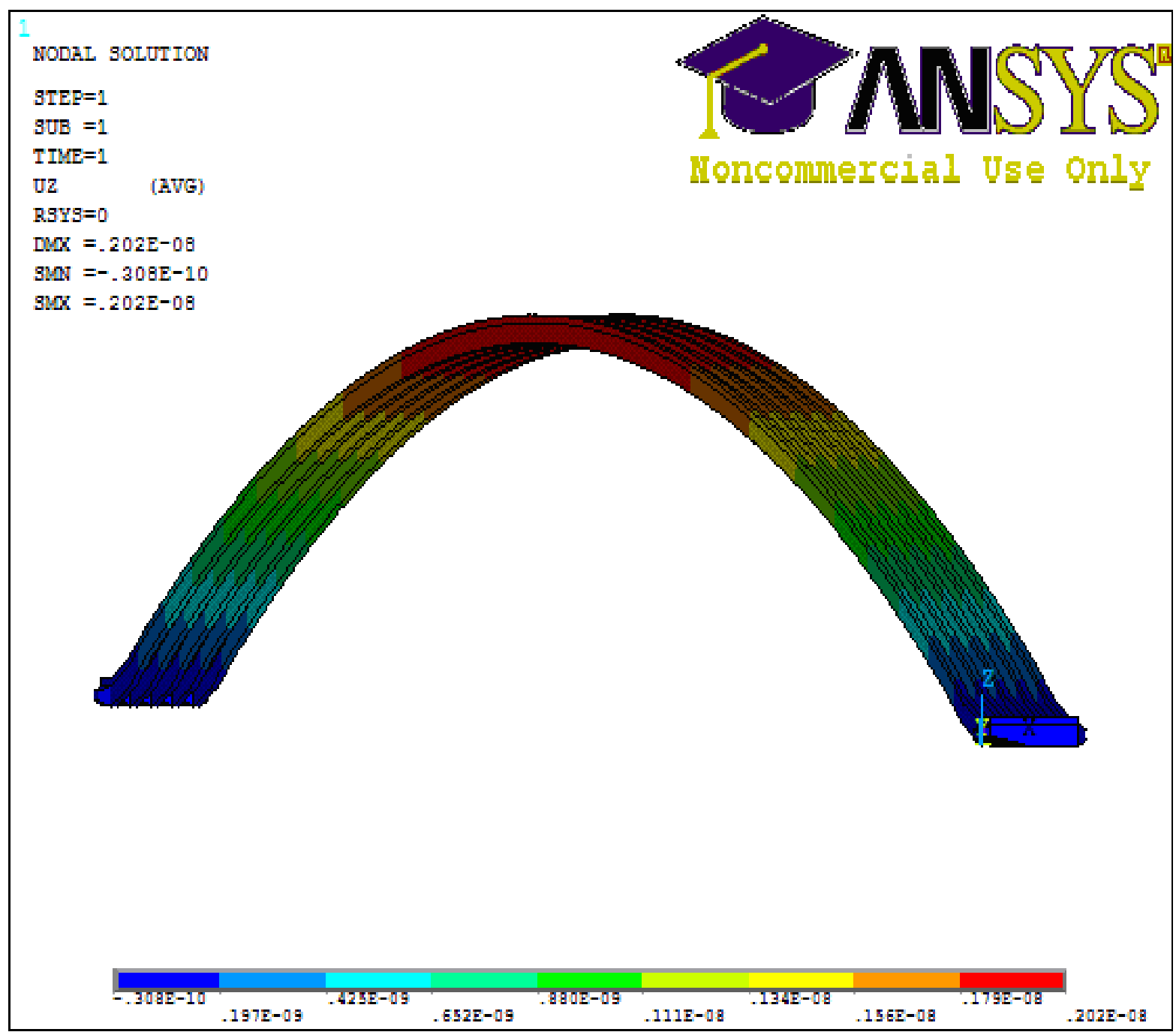

Figure 3. $33 \mathrm{Z}$-Deflection of 1D PhC with application of $10 \mathrm{~V}$ on the 1-D PhC with electrode . 


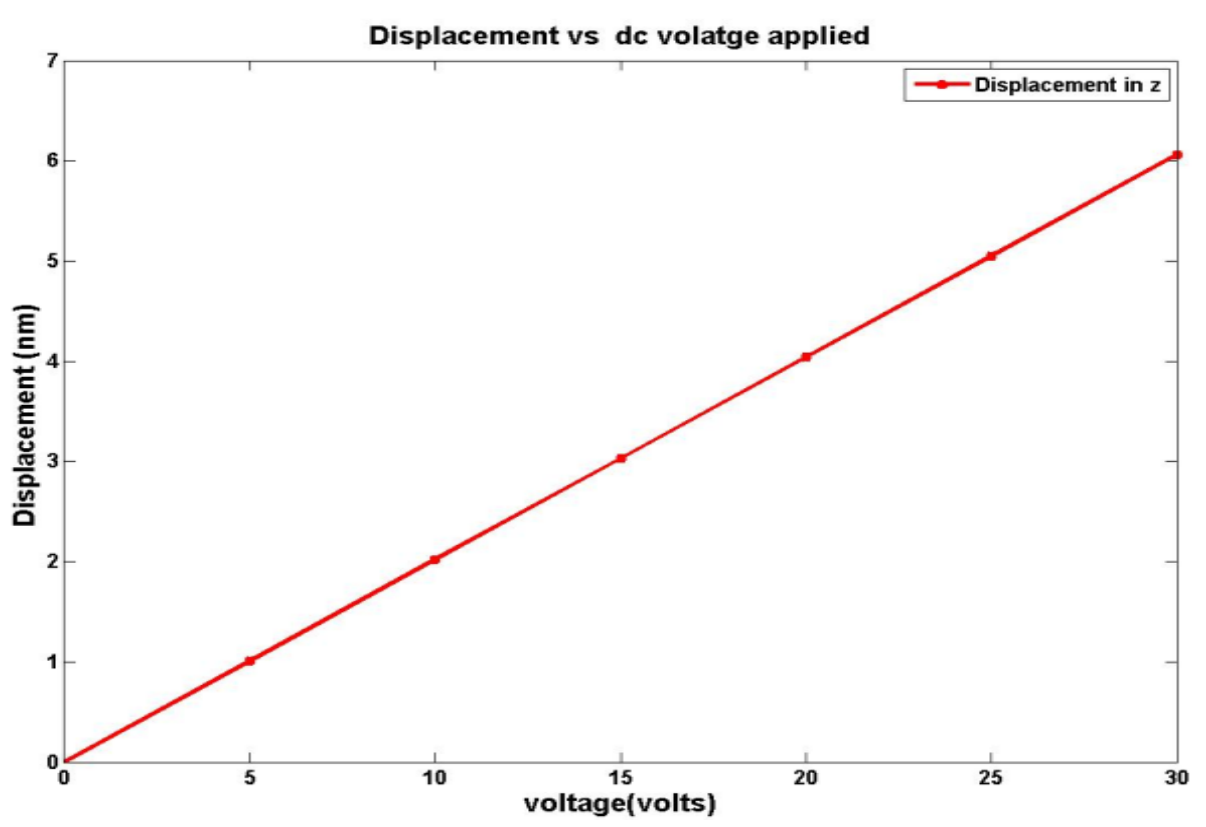

Figure 3. 34 Deflection of 1-D PhC in Z-direction vs. voltage

Figure 3.14 shows the results of deformation in a 1D PhC with lattice stripes anchored on both ends; using $200 \mathrm{~nm}$ thick metal which was used as a conductive electrode as shown in Figure3.13. This structural arrangement induces a deflection which is much larger than direct parameter tuning (lattice spacing, etc), and offers a method of indirect bandgap tuning, as mentioned in the literature ${ }^{46,47,48,49}$. Voltage is varied to find the relationship between applied potential and deflection. It is noted that the modeling tool does not take into account the breakdown voltage of the piezoelectric material. Hence, optimum values of voltage are used to find the voltage vs. deflection relationship, as shown in Figure 3.15. As the voltage is varied from 0 to $30 \mathrm{~V}$, deflection increased from 0 to $6 \mathrm{~nm}$. Maximum deflection is experienced in the z-direction compared to $\mathrm{x}$ - and $\mathrm{y}$-directions, which are of the order of $0.1 \mathrm{~nm}$. However this z-axis deflection does not cause a significant change in $h$, but does potentially change the effective angle of incidence of light interacting with the $\mathrm{PhC}$ lattice.

Experimental measurements are needed to determine the impact of this deflection on optical properties.

\subsubsection{2(b) AC Analysis}

AC Analysis was performed on the initial designs to determine whether or not higher levels of deformation are possible if excitation is provided near a structural resonance. 


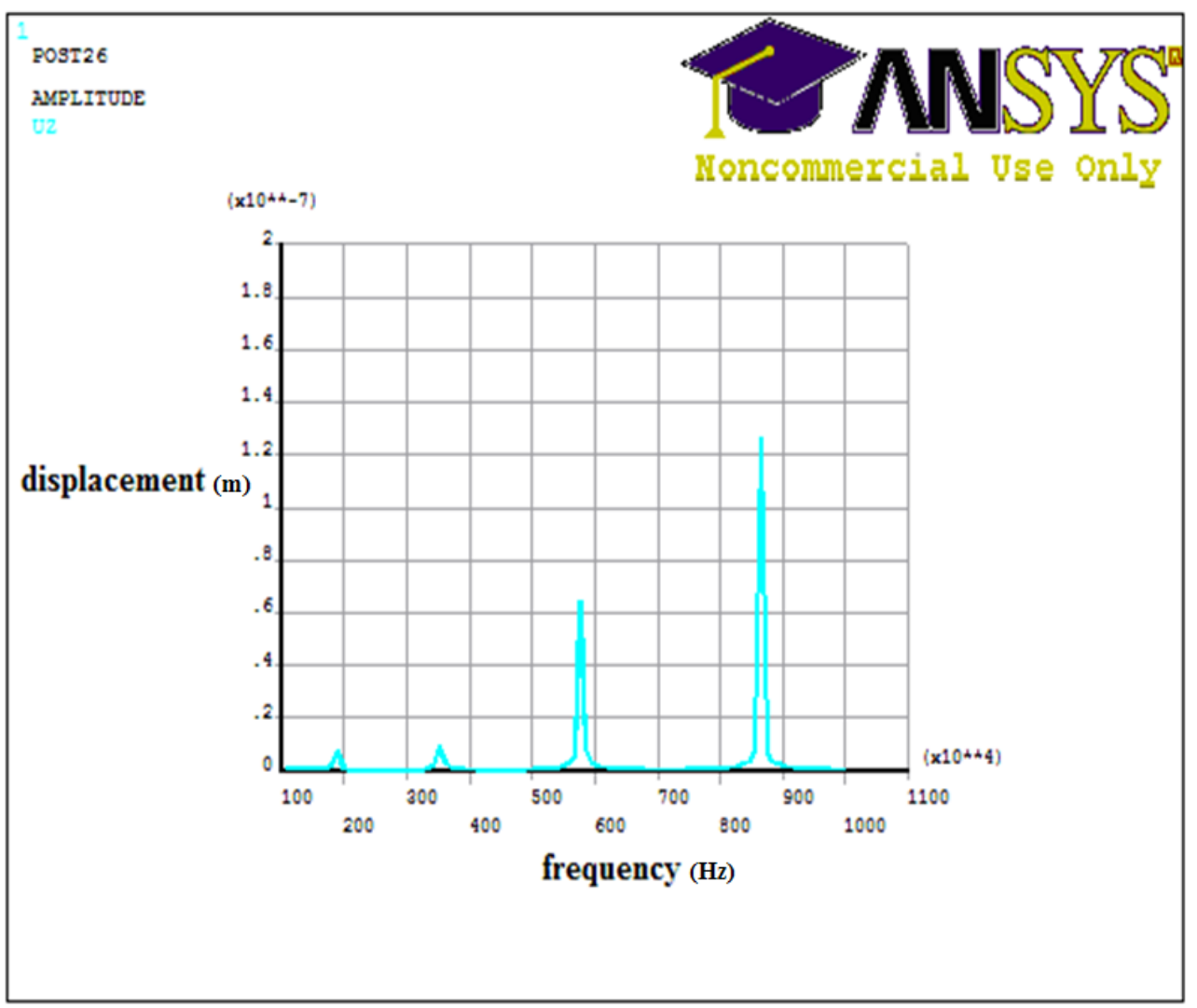

Figure 3. 35 Deflection in Z-direction vs. frequency

Harmonic FEA was performed on the PhC lattice structure to obtain information about deflection near resonance. As the frequency is varied from $1 \mathrm{MHz}$ to $10 \mathrm{MHz}$, the beam experiences resonance near frequencies of $1.9 \mathrm{MHz}$, 3.5MHz, $5.8 \mathrm{MHz}, 8.7 \mathrm{MHz}$ as shown in Figure 3.17. Higher magnitudes of deflection are generated at higher resonance harmonics, where larger magnitudes of deflection of the beam deflection are obtained. All simulations were performed with the surrounding media defined as vacuum. However, experimentally deflections might be less than the theoretical ones due to air and material damping.

\subsection{MPB Modeling for Polycrystalline PhC structures in GaN}

MPB modeling was performed to verify the functional differences of polycrystalline PhCs in $\mathrm{GaN}^{50}$ which were considered to be different from the ideal planar PhCs. The differences between polycrystalline PhCs compared to ideal $\mathrm{PhCs}$ are (1) finite lattice domains of varying sizes, and (2) adjacent lattice domains which are placed at different angles with each other. 
(1)Finite Lattice domains: MPB simulations were performed to observe the variation of bandgaps with different lattice domain sizes.
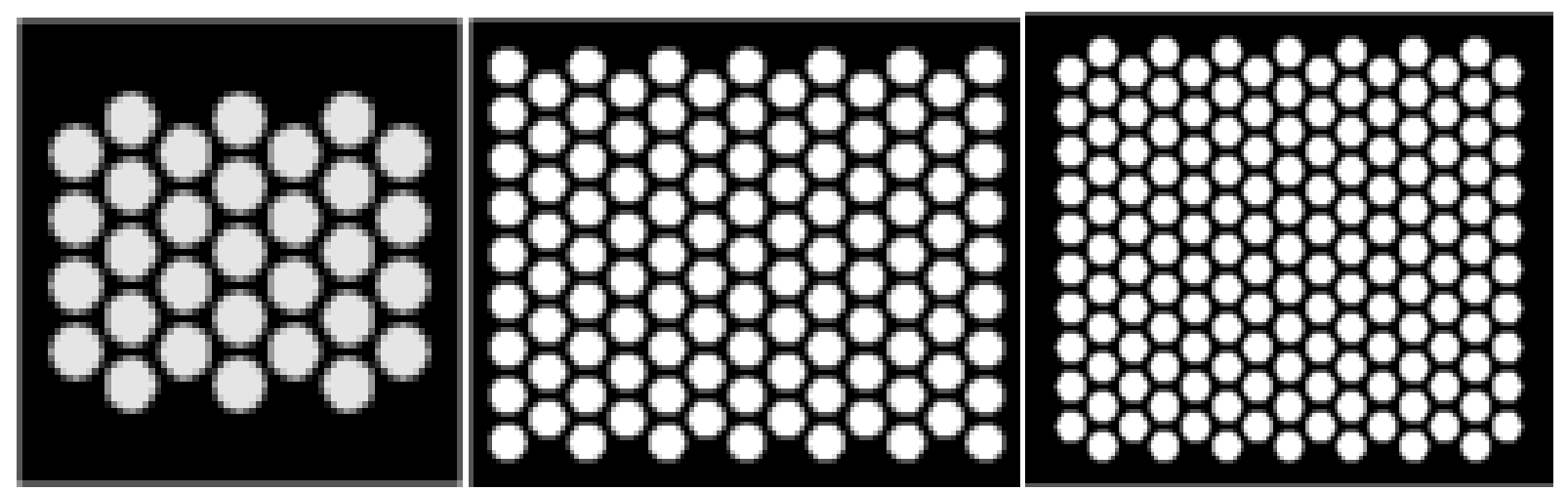

Figure 3. 36 (a) lattice domain with 7X9arrays of holes (b)lattice domain with 13X17 arrays of holes(c)17X29arrays of holes arranged in triangular lattice on GaN

Figure 3.18 shows the lattice domains of different sizes which were simulated using MPB to find the bandgap variation. Figure 3.19 represents the bandgap variations found with differently sized lattice domains. From the graph it can be inferred that, as the lattice domain size increases, TE bandgap increases, whereas TM bandgap decreases. As one reaches an ideal photonic crystal of infinite size TE bandgap sees its maximum whereas TM bandgap reaches its minimum.

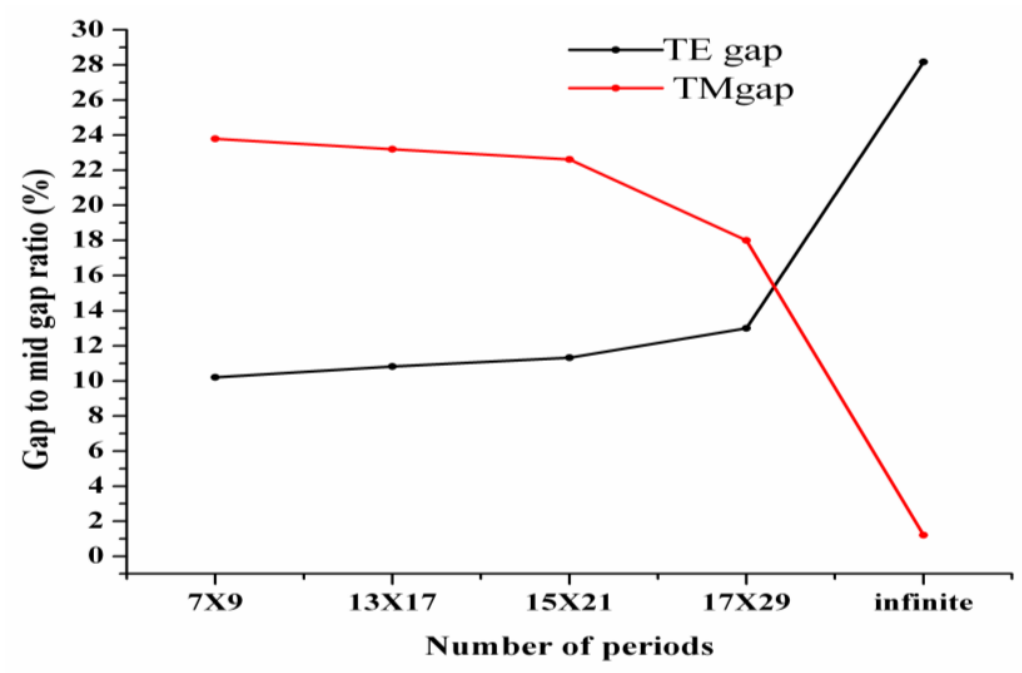

Figure 3. 37 Gap-to-mid-gap ratio vs. different sizes of lattice domains

.According to the literature a minimum of 6 to 10 periods are necessary to create an appreciable bandgap ${ }^{51,52,53,54}$. The bandgap gradually closes for sizes smaller than $7 \times 9$ lattice hole arrays, which is consistent with experimental and theoretical results presented in the literature ${ }^{15,16}$. Lattice domains observed in the nanosphere deposition 
performed under the supervision of Dr. Korakakis(WVU) were typically $7 \times 9$ periods in size which showed appreciable bandgap in the simulations. Results also showed that a bandgap existed for larger domains of PhC lattices.

(2)Dependency of bandgap on oblique angle of incidence: For periodic optical structures, K-space coordinates are the Fourier transform representation of Cartesian coordinates arising due to the periodic Bloch optical modes of the structure. Variation of angle of incidence in Cartesian crystal geometry implies variation of K points in K-space.

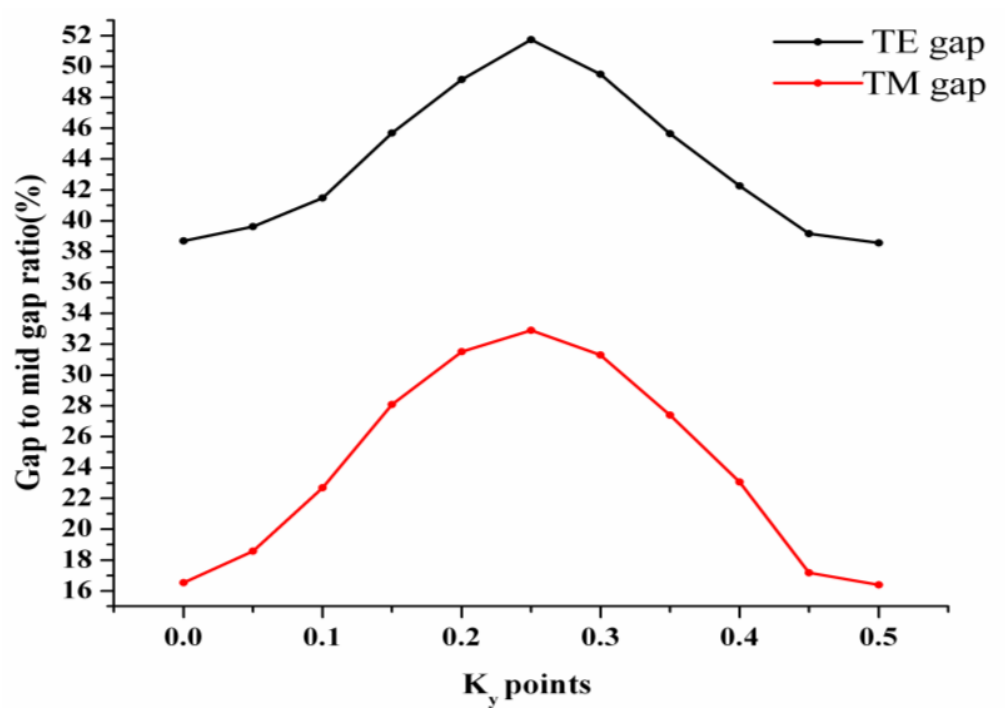

Figure 3. 38 Bandgap vs. angle of incidence (variation of $K$ points)

From Figure 3.20, we can see that as the K-points (angle of incidence) are varied from 0 to 0.5 (effectively 0 to 90 degrees) in the irreducible Brillourin zone, the bandgap (both TE and TM) increase and decrease symmetrically, both following the same pattern. As the irreducible Brillourin zone describes optical behavior in the whole crystal, the pattern of bandgap variation shown in Figure 3.20 will repeat for light propagation angles from 90-360 degrees. The fabricated photonic crystal has adjacent lattice domains that meet at varying angles.

(3) Adjacent domains placed at different angles: Simulations were performed to determine the bandgap caused by two adjacent domains, one of which is at a 45 degree angle to the other with different domain sizes as shown in Figure 3.21(a,b,c,d). 

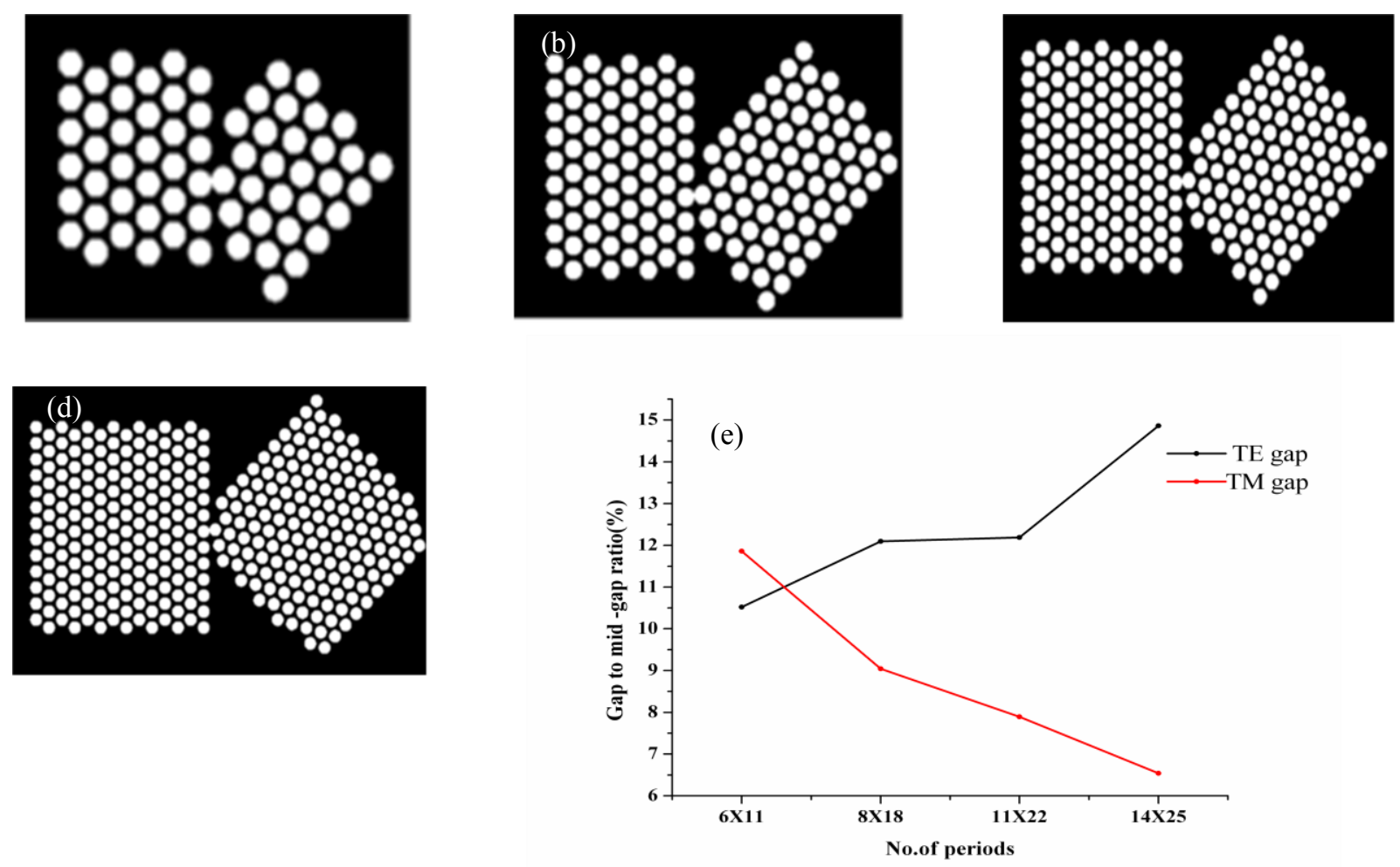

Figure 3. 39(a,b,c,d) Adjacent domains placed at an angle of $45^{\circ}$ to each other with different lattice domain sizes (e) Gapto-midgap ratio vs. different lattice domains sizes of adjacent $\mathrm{PhCs}$ placed at an angle of $45^{\circ}$ to each other

Figure 3.21(e) indicates that, as the lattice domain sizes of adjacently placed lattices vary, variation in the bandgap can be observed. From the graph, it can be inferred that, with the increase in the size of lattice domains, TE gap increases, accompanied by a similar decrease in TM gap. It indicates that some level of bandgap will exist in either the TE or TM modes for all angles of propagation in the plane of the crystal and for any angle variation between adjacent lattice domains.

\subsection{Waveguide Modeling}

The MPB software tool was used to demonstrate the existence of an optimal bandgap for a 2-D PhC of triangular lattice of air holes in GaN which has a normalized slab thickness $(h / a)$ of 0.8 and lattice hole radius $\left(r_{l a l} / a\right)$ of 0.29 . The dimensions derived were used for fabricating a 2-D PhC which operates in visible spectral range. However, the device functionality may fail if the quality of GaN fabricated in the lab is not optimal or if the GaN slab doesn't act as a waveguide. Initially, the wave guiding properties of $\mathrm{GaN}$ on sapphire substrate with specified thickness and refractive index were modeled using Opti-BPM. Opti-BPM ${ }^{55}$ is used to investigate linear and non-linear properties of light propagation in waveguides. In BPM, it is assumed that the device has an optical axis and that most of the light travels in the axis direction (paraxial approximation). 
After determining the waveguide geometry in the modeling, an input plane was drawn. Because a prism coupler would be used for experimental measurements, a modal field was chosen as input wave for the simulations. More details about the input types and the assumptions in the simulation software can be found in the documentation of Opti-Wave software ${ }^{20}$. The structure can be simulated either in 2-D mode or in 3-D mode. After the simulation, the refractive index profile and the field distribution obtained are shown in the Figure 5.2. 2-D (or 3-D) mode solver can be used to determine the number of modes that can exist and their corresponding modal indices in the structure. Scanning the parameters such as thickness, refractive index, wavelength can be very useful in finding the optimized waveguide structure.
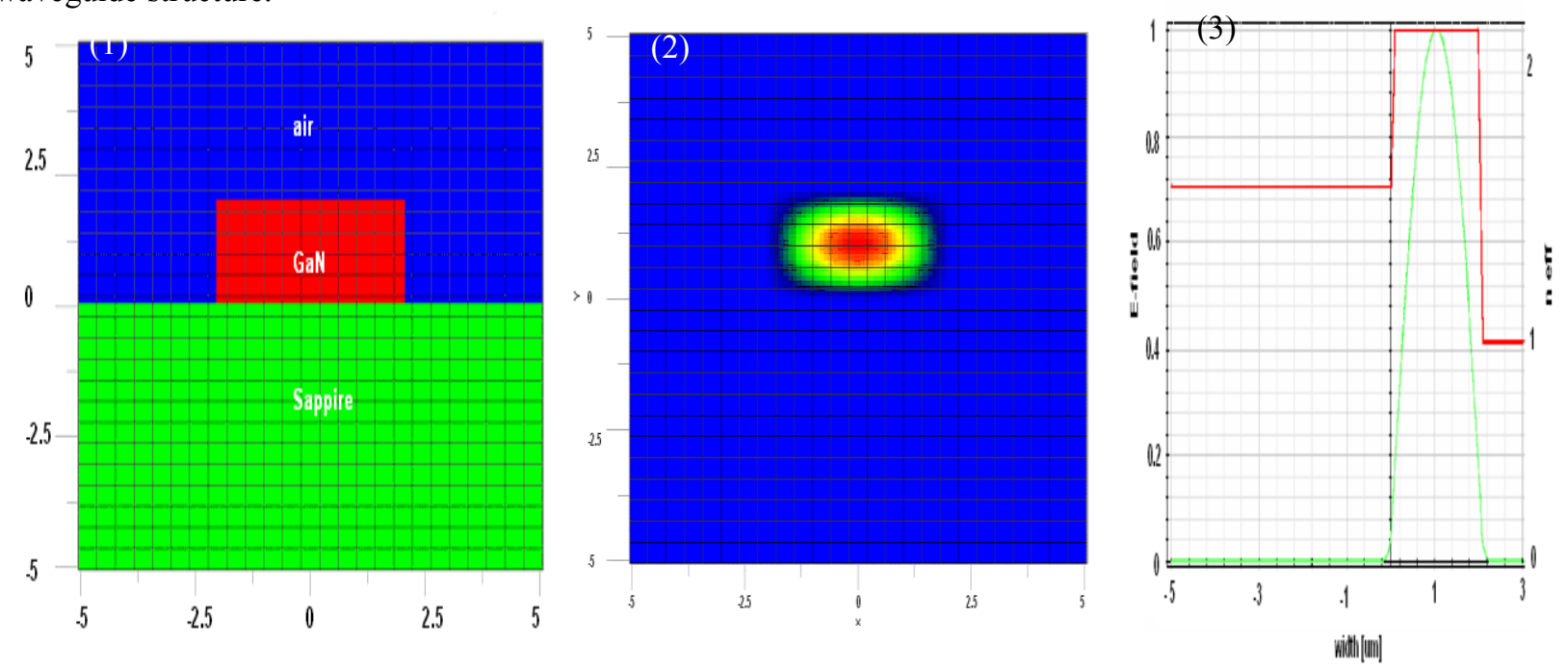

Figure 5. 20(1) GaN waveguide on top of a thin AlN layer and sapphire substrate. (2) The field intensity as modeled by OptBPM that shows a complete confinement within the waveguide (3) Field intensity (green) plotted across the different refractive indices of the layers (red.)

The refractive index profile, as shown in Figure 5.20 was a representation of a GaN (Gallium nitride) waveguide with a small buffer layer of $\operatorname{AlN}(\sim 61 \mathrm{~nm})$ grown on a sapphire substrate under the supervision of Dr. D. Korakakis (WVU). Full confinement of the wave in GaN is shown in Figure 5.20, indicating that GaN on sapphire can be used as a waveguide. The refractive indices and the thicknesses that were used in modeling were measured by ellipsometry. The thicknesses of substrate and the cladding (air) were assumed to be small in this model in order to speed up the simulation. 

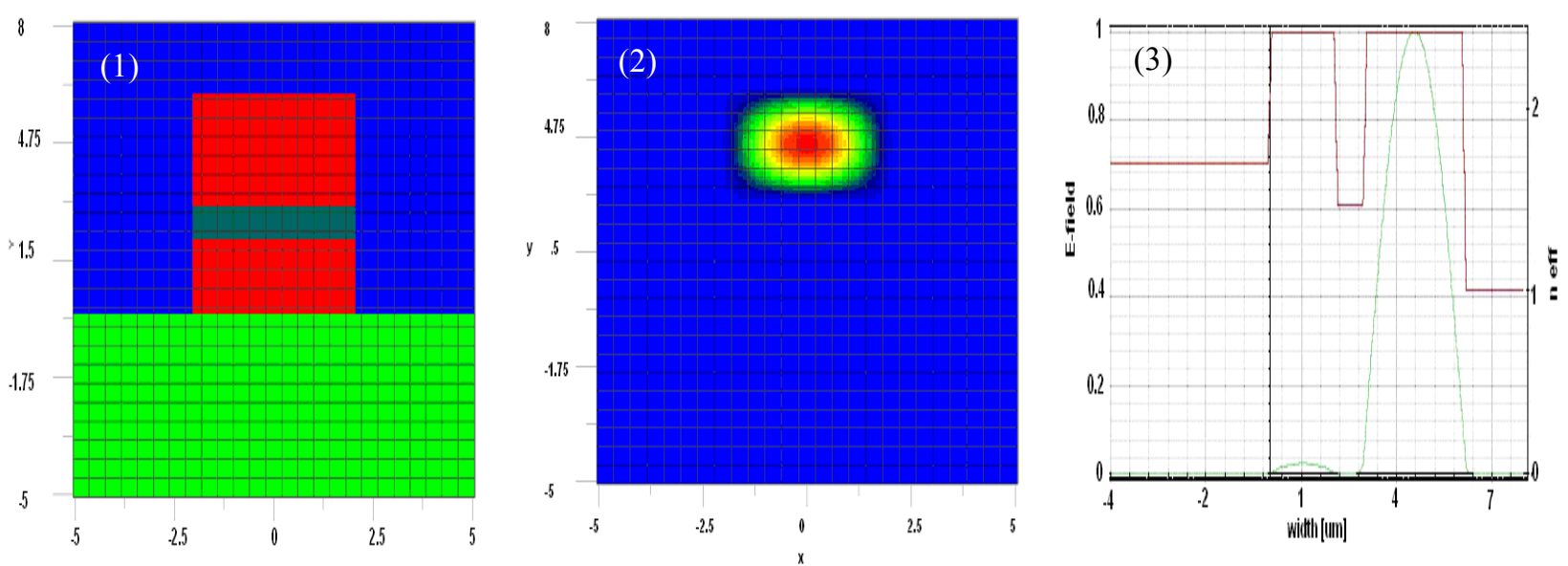

Figure 5. 21 (1) The GaN waveguide with an underlying $\mathrm{SiO}_{2}$ layer which will eventually be removed after the fabrication process is finished and obtain a flow channel instead. (2) The field intensity demonstrates the confinement of the light in the waveguiding GaN layer that will include the PhC structure. (3) Field intensity (green) plotted across the different refractive indices of the layers (red )

In order to implement a 2-D PhC fabricated on a $\mathrm{GaN}$ slab as a transducer of a biosensor, a suspended structure is most optimal. One potential device architecture employs a $\mathrm{SiO}_{2}$ layer as a sacrificial layer that will be removed to create a suspended structure. Coupling of light into the 2-D $\mathrm{PhC}$ slab above $\mathrm{SiO}_{2}$ is very crucial to operate the same as a biosensor. Therefore, modeling efforts were done to find optimum slab thickness of GaN required to couple most of the light into the top waveguiding layer. OptiBPM shows that a full confinement can be obtained when the top GaN layer (above the flow channel) is at least $1 \mu \mathrm{m}$ thicker than the lower GaN layer.

\subsection{SUMMARY}

From theoretical results of the optical modeling of PhCs it was observed that, as thickness is varied by $0.1 a$, there is a bandgap change of nearly $2-4 \%$. The same is observed while varying the width of the grating. However, in FEA studies, the deformation of AlN films with applied voltages within the breakdown limit of the material lead to deformations on the order of picometers, which are not large enough to affect the optical bandgap. By applying higher voltages and by varying frequency, a maximum slab deflection of $120 \mathrm{~nm}$ can be achieved. A $7 \mathrm{~nm}$ deflection is sufficient for creating a $2-4 \%$ change in the bandgap, which can be acquired by applying a voltage of $30 \mathrm{~V}$. Bandgap can be actively tuned by following this technique. 


\section{CHAPTER 4: FABRICATION}

\subsection{Introduction}

Modeling results indicated that high deformation can be observed for a suspended 1-D PhCs in AlN which can eventually be used for tuning the bandgap. Si substrate was considered for initial process development of fabricating nanometer-scale 1-D PhCs and for optical characterization. Due to the limitations on the equipment to mechanically characterize a nanometer scale 1-D PhC, efforts were also made to fabricate micron-scale suspended 1-D PhCs in AlN for initial tuning measurements.

\subsection{Fabrication of 1-D PhCs for optical characterization}

\subsubsection{Nanometer scale 1-D PhCs in Si}

Optical Characterization of 1-D PhCs required nanometer scale PhC patterns due to the limitations on the operating wavelengths of the characterization equipment. Because of this, nanometer patterns were fabricated using E-beam lithography techniques. Initially, efforts were made to etch PhCs into Si for the reason that Si processing is relatively easy when compared to AlN. The process flow for fabricating 1-D PhCs in Si and SOI substrates were shown in Figure 4.1.

1. Degrease the sample

2. Spin ma-N2403 for $50 \mathrm{sec}$ at 4500 rpm \& Soft bake 1 min at $95^{\circ} \mathrm{C}$

3. Pattern with E-beam
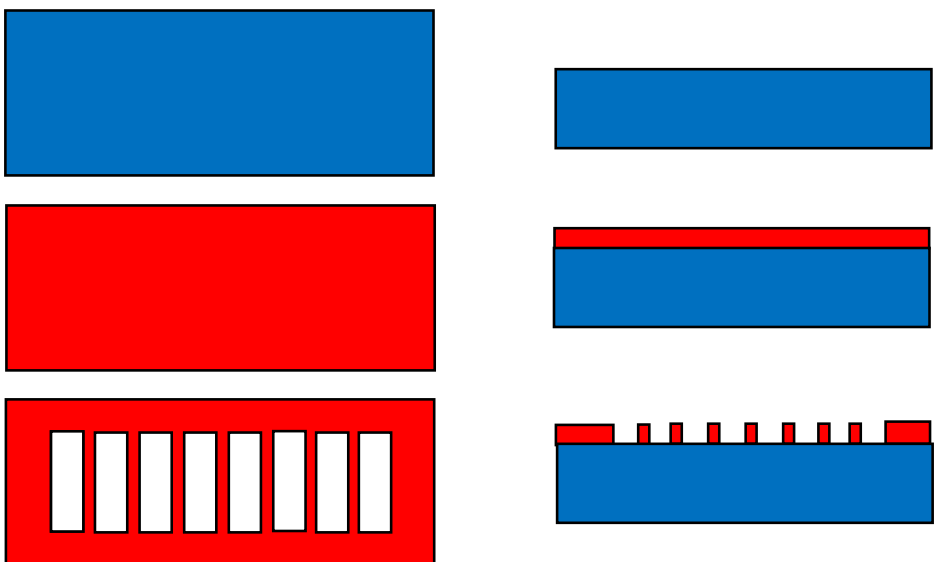

Lithography and developed in $300 \mathrm{MIF}$ for $16 \mathrm{sec}$
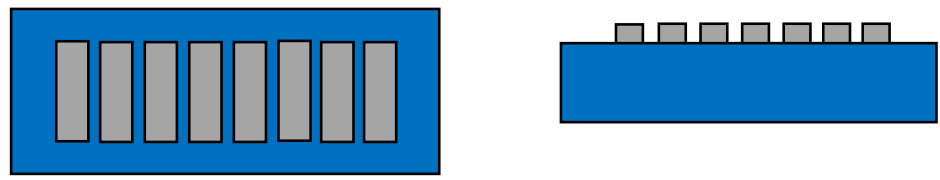

4. Metal liftoff with Nickel $(\sim 20 \mathrm{~nm})$
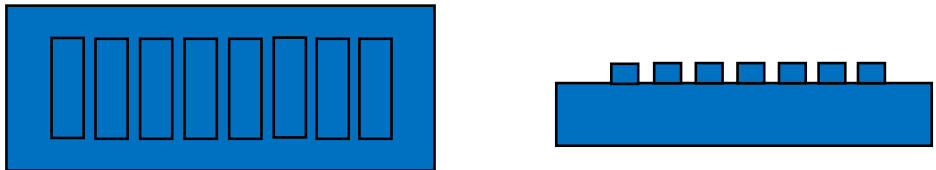

Figure 4. 2 Process flow for fabricating nanometer scale 1-D PhCs in Si and SOI 
The substrate was thoroughly cleaned with acetone and methanol for 5 mins each followed by a HF dip to remove any oxide on the surface. DI water is used to rinse the samples. A nitrogen blower was used to blow off any water on the samples. The samples were then kept in an oven for nearly $30 \mathrm{~min}$ at a temperature of $100^{\circ} \mathrm{C}$ to remove any moisture on the samples. ma-N 2403, a negative E-beam resist was spun on the samples at4500rpm for 50sec followed by a soft bake for $1 \mathrm{~min}$.

On one of the edges of the sample, a small scratch was made on the resist before loading the sample into JEOL system. This scratch can be used to focus the beam into a very small diameter which is required for writing high quality nanometer scale features. Two different 1-D PhC patterns were designed using Design CAD LT 2000 which were used by the NPGS software to give commands to E-beam lithography system for pattern writing. In order to write patterns at a working distance of $8 \mathrm{~mm}$, a dose of $80 \mu \mathrm{C} / \mathrm{cm}^{2}$, magnification scale of $1100 x$ and $40 \mathrm{pA}$ probe current was used. The samples were unloaded after pattern writing and developed in 300MIF developer for 16seconds. Nickel with thickness $\sim 30 \mathrm{~nm}$ was sputtered on the samples in order to use the same as etch mask. The nanometer patterns were transferred into Si using 400/100Watts ICP/RIE power, 20mT pressure, 24/6 sccm of $\mathrm{CF}_{4} / \mathrm{O}_{2}$ gases in Trion etch system. Tthe SEM images are shown below .SEM Images 01:1-D PhC patterns in Si

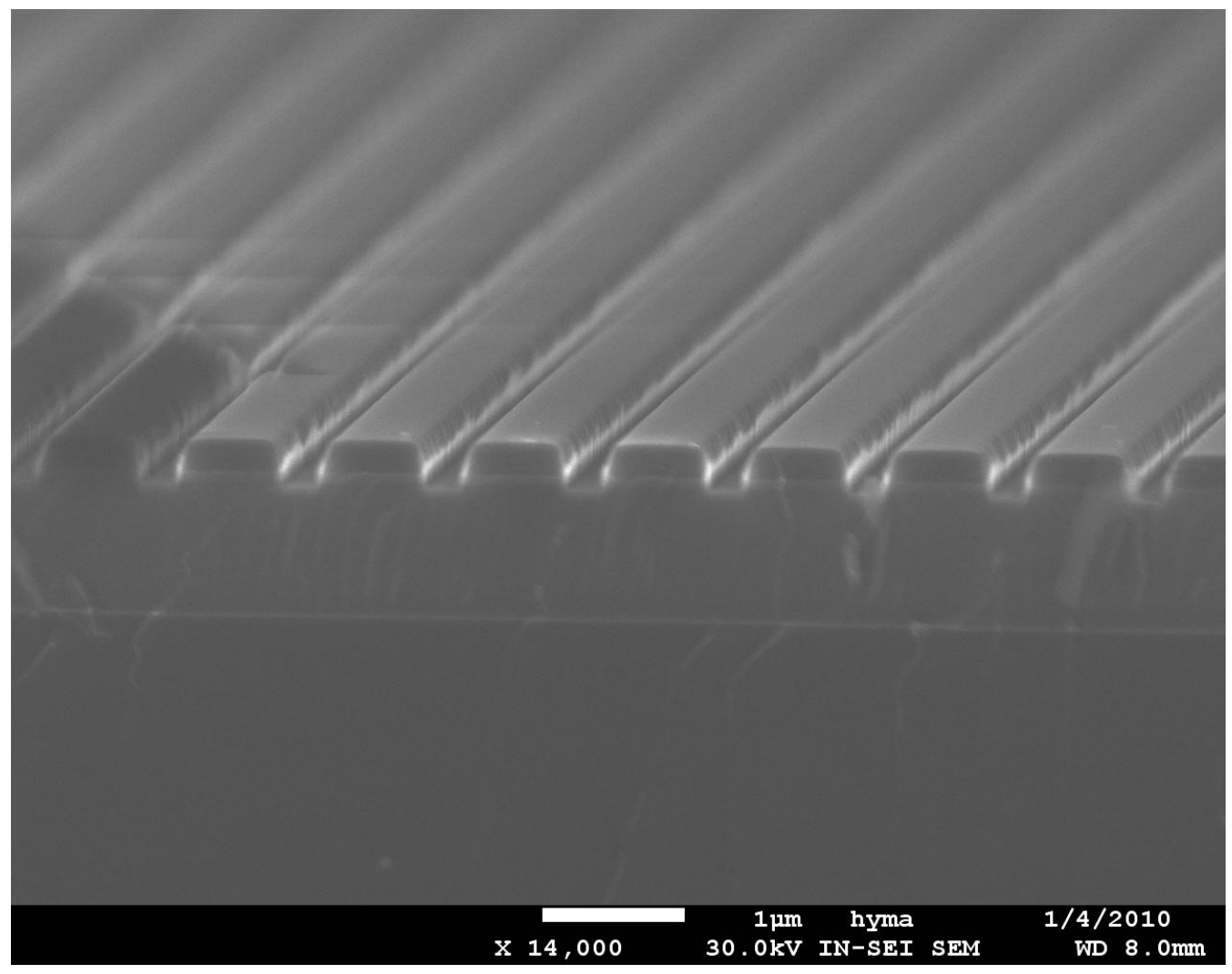

Figure 4. 2 1-D PhC pattern with 700nm wide Si stripe with 300nm air spacing on SOI samples 


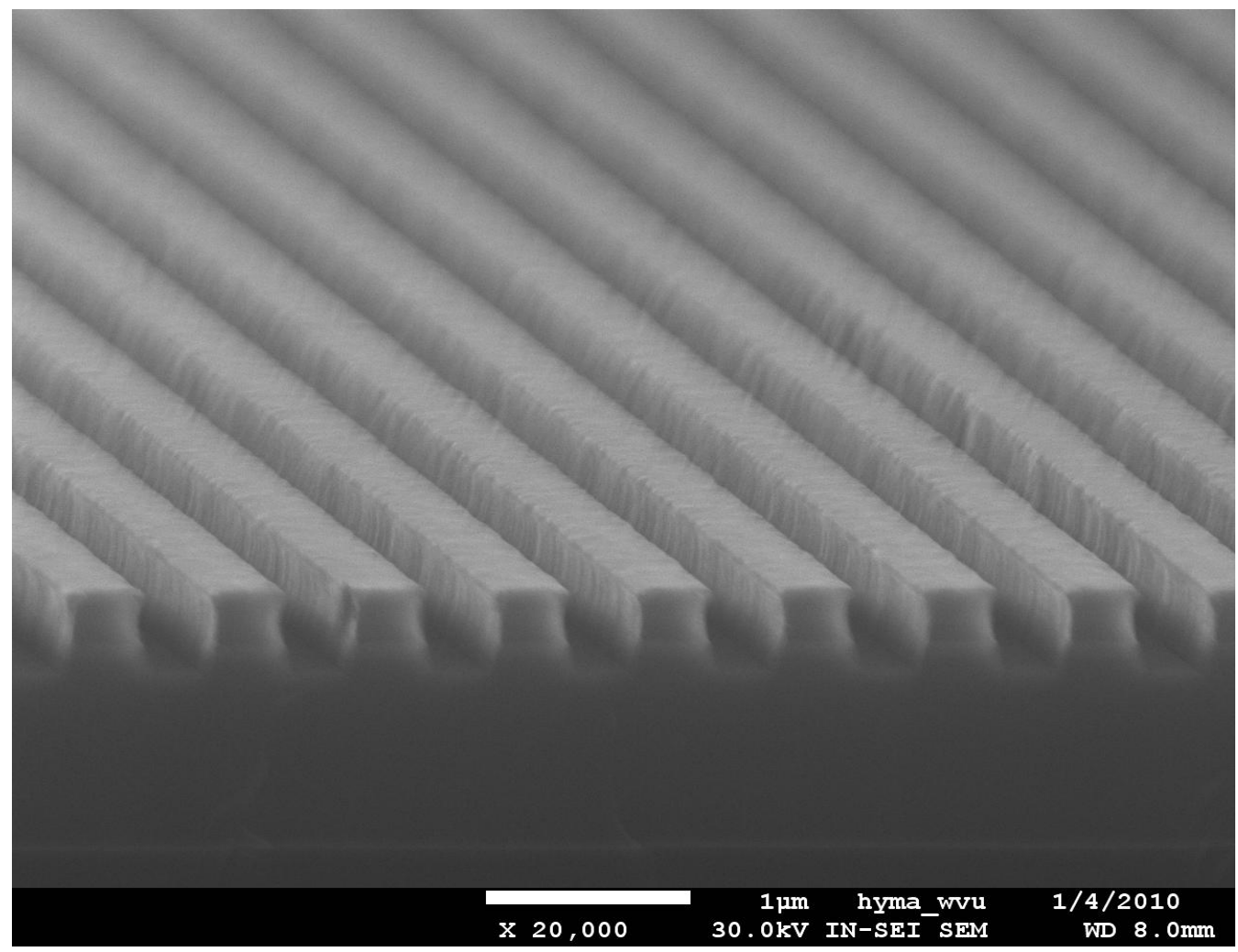

Figure 4. 3 1-D PhCs pattern with 400nm wide Si stripe with 300nm air spacing on SOI samples. Samples were over etched into $\mathrm{SiO}_{2}$ in the image

\subsubsection{Introduction to Aluminum Nitride:}

Aluminum nitride (AIN) has a wurtzite crystal structure which gained significance in MEMs device fabrication because of its piezoelectric properties. It exhibits high thermal stability which makes it an ideal candidate in high temperature applications. Its high bandgap $(\sim 6.2 \mathrm{eV})$ and refractive index $(\sim 2.11)$ make it an ideal candidate for optoelectronic applications. AlN thin films can be obtained using various techniques such as CVD (chemical vapor deposition), sputtering, vapor phase epitaxy or molecular beam epitaxy (MBE). Of these techniques, sputtering is a low cost, versatile technique that is widely used to fabricate AlN thin films. Sputtered AlN thin films are produced under much lower temperatures compared to MOVPE. Sputtered AIN is polycrystalline, whereas MOVPE grown AlN is single crystalline. Sputtered AIN exhibits a reduced amount of piezoelectricity compared to MOVPE grown AlN because of the random orientation of the crystal domains. Degreasing the sample thoroughly and pre-sputtering for a reasonable amount of time to remove contamination on the surface of target material improves the quality of sputtered AlN thin films. 


\subsubsection{Etching of AIN}

1-D PhCs in reality can be called quasi PhCs that have a slab structure made up of lattice 'lines.' The fabrication of this type of structure requires resist patterning and subsequent transfer of patterns to the slab material by etching. Different etching techniques, such as wet and dry etching, can be considered depending on different parameters such as etch rate, selectivity and isotropy. Etch rates depend on various factors including the quality of thin films. To etch

sputtered amorphous AlN, different acidic and basic solutions such as $\mathrm{HF} / \mathrm{H}_{2} 0^{56}, \mathrm{HF} / \mathrm{HNO}_{3}{ }^{57}, \mathrm{KOH}^{58}$ or $\mathrm{NaOH}^{59}$ can be used. It was reported that AZ400K developer whose active ingredient is $\mathrm{KOH}$ can also produce substantial etching of polycrystalline $\mathrm{AlN}$ which is selective over $\mathrm{GaN}$ and $\mathrm{Al}_{2} \mathrm{O}_{3}{ }^{60}$.In the reported work, the time dependence of etch rate, and also the undercut caused by etching the samples in heated AZ400K solution were measured. It was reported that isotropic etching in AZ400K increases with increase in temperature. Generally, wet etching is more selective and isotropic whereas dry etching is more anisotropic. Anisotropic etching is optimal in $\mathrm{PhC}$ pattern transfer with very small feature sizes. Isotropic etching often results in deformed lattice structures which results in improper device functionality. Various plasma etch techniques such as reactive ion etching (RIE), electron cyclotron resonance (ECR), inductively coupled plasma (ICP), and reactive ion beam etching (RIBE) have been used to etch III-nitrides ${ }^{6}$. In an ICP etching technique, high density plasma is created by applying rf-power to an inductive coil encircling a dielectric vessel. The coil produces an electric field which induces a strong magnetic field in the vertical plane, trapping electrons in the center of the chamber thereby producing high density plasma. At pressures lower than $20 \mathrm{mT}$, the plasma diffuses from the center of the chamber to the substrate at low ion energy, producing minimal damage and high etch rates. ICP etch systems produce the highest etch rates and smoothest morphologies in group-III nitrides. High-plasma-density systems improve the bond-breaking efficiency of the etch process and enhance sputter desorption of etch products from the surface of the material. While ICP etch characteristics are similar to ECR, ICP systems are more widely used as they are easier to scale-up and are more economical ${ }^{61}$.

\subsubsection{Nanometer scale 1-D PhCs in AIN}

After successful fabrication of 1-D PhCs in $\mathrm{Si}$, efforts were made to fabricate nanometer scale 1-D PhCs in AlN on a Si substrate. The process flow and the SEM images are shown below in Figure 4.4. 
1. Degrease the sample

2. Sputter AlN
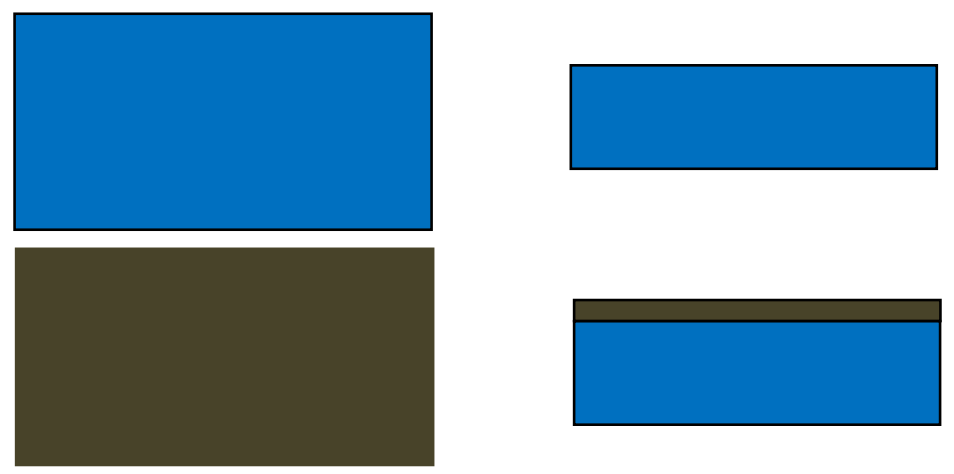

3. Deposit $\mathrm{SiO}_{2}$
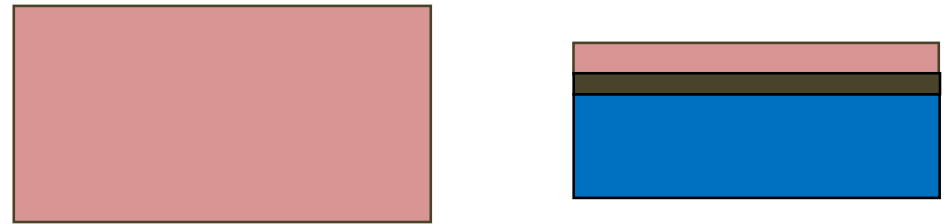

4. Spin ma-N2403and soft bake
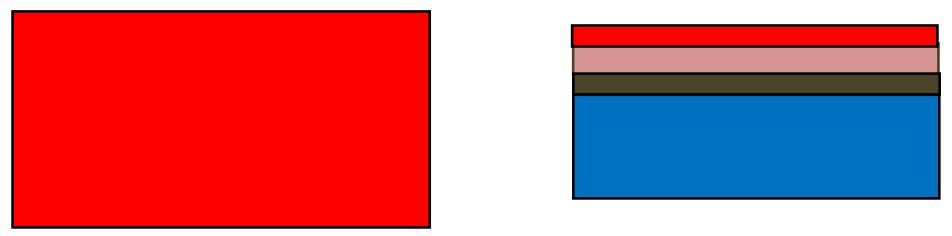

5. Develop patterns in AZ300MIF developer.
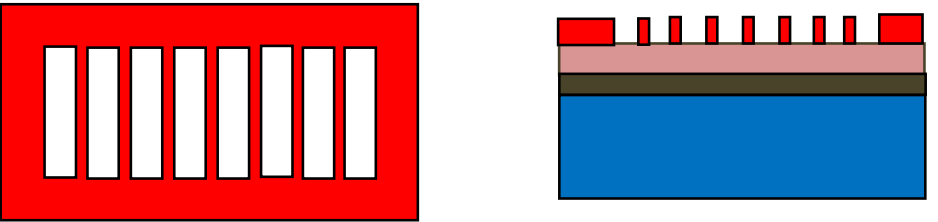

6. Metal liftoff with Nickel $\sim 50 \mathrm{~nm}$

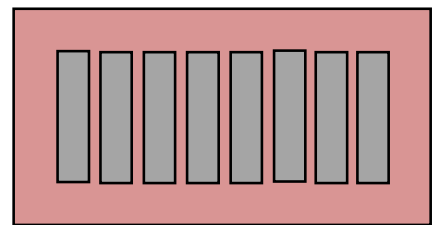

$\sqcap \sqcap \sqcap \sqcap \sqcap \sqcap \sqcap$
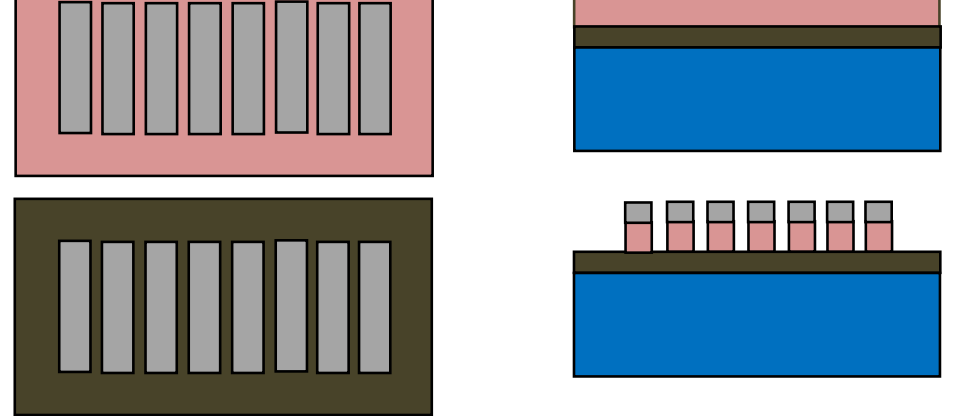

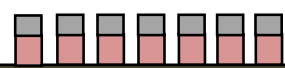

7. Etch $\mathrm{SiO}_{2}$ using $\mathrm{CF}_{4} / \mathrm{O}_{2}$ plasma.

8. Etch AlNusing $\mathrm{Cl}_{2}$ plasma.

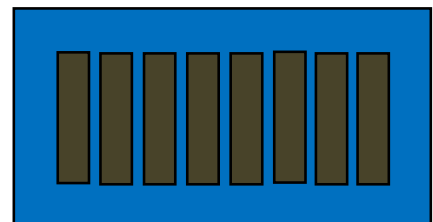

$\sqcap \square \square \square \square \square$

Figure 4. 4 Process flow for fabrication of 1-DPhC in AlN 
The Si samples were cleaned thoroughly and AlN films were sputter deposited on Si by using CVC-610 Direct Current (DC) magnetron sputtering system in an $\mathrm{Ar} / \mathrm{N}_{2}$ environment. Pre-sputtering the Al target to clean and equilibrate the target surface prior to film deposition reduces the chance of film contamination ${ }^{62}$. Approximately $200 \mathrm{~nm}$ thick AlN is sputtered using a 3" $\mathrm{Al}$ target which is $99.99 \%$ pure with a $\mathrm{Ar} / \mathrm{N}_{2}$ gas concentration of $3 / 27 \mathrm{sccm}$ and $45 \mathrm{mT}$ pressure at room temperature. AlN is relatively hard to etch because of its atomic bond strength. In order to etch $200 \mathrm{~nm}$ of AlN, a very thick etch mask is desired. But, due to the limitations in the liftoff technique (very thin e-beam resist; $\sim 250 \mathrm{~nm}$ ), only 40-50nm of etch mask can be used. Even though Ni is considered a hard etch mask, $40-50 \mathrm{~nm}$ of $\mathrm{Ni}$ is not sufficient to etch $200 \mathrm{~nm}$ of AlN in a chlorine plasma. So, $\mathrm{SiO}_{2}$ is deposited on the samples using PECVD (Plasma enhanced chemical vapor deposition) after AlN deposition. ma-N2403, e-beam resist was spun@4500rpm for 50sec followed by 1 minute soft bake at $95^{\circ} \mathrm{C}$. The patterns were then written using e-beam lithography technique. The samples were developed in AZ300MIF for 35sec. Ni was deposited on the pattern with thickness of $\sim 50 \mathrm{~nm}$. Liftoff was performed on the samples. Etching is performed on a Trion Mini-Lock Phantom III Series ICP-RIE system. A chlorine based gas mixture was used for dry-etching $\mathrm{AlN}$. In a $\mathrm{Cl}_{2} / \mathrm{BCl}_{3}$ plasma, the excited material interacts with the exposed regions of the material while the ions in the plasma physically bombard the sample and remove material. The vertical etch rate depends on both the chemical and physical etch but the etch rate is not the sum of the two quantities. The etch mask was used to transfer the patterns to $\mathrm{SiO}_{2}$ using $\mathrm{CF}_{4} / \mathrm{O}_{2}$ plasma. Then $\mathrm{SiO}_{2}$ etch mask along with the remaining $\mathrm{Ni}$ was used to transfer patterns to AlN using $12 / 18 \mathrm{sccm}$ of $\mathrm{BCl}_{3} / \mathrm{Cl}_{2}$ plasma in $400 / 100 \mathrm{ICP} / \mathrm{RIE}$ power at $10 \mathrm{mT}$ pressure. The SEM images at each step of fabrication were shown in Figure4. $(5,6,7,8)$ 


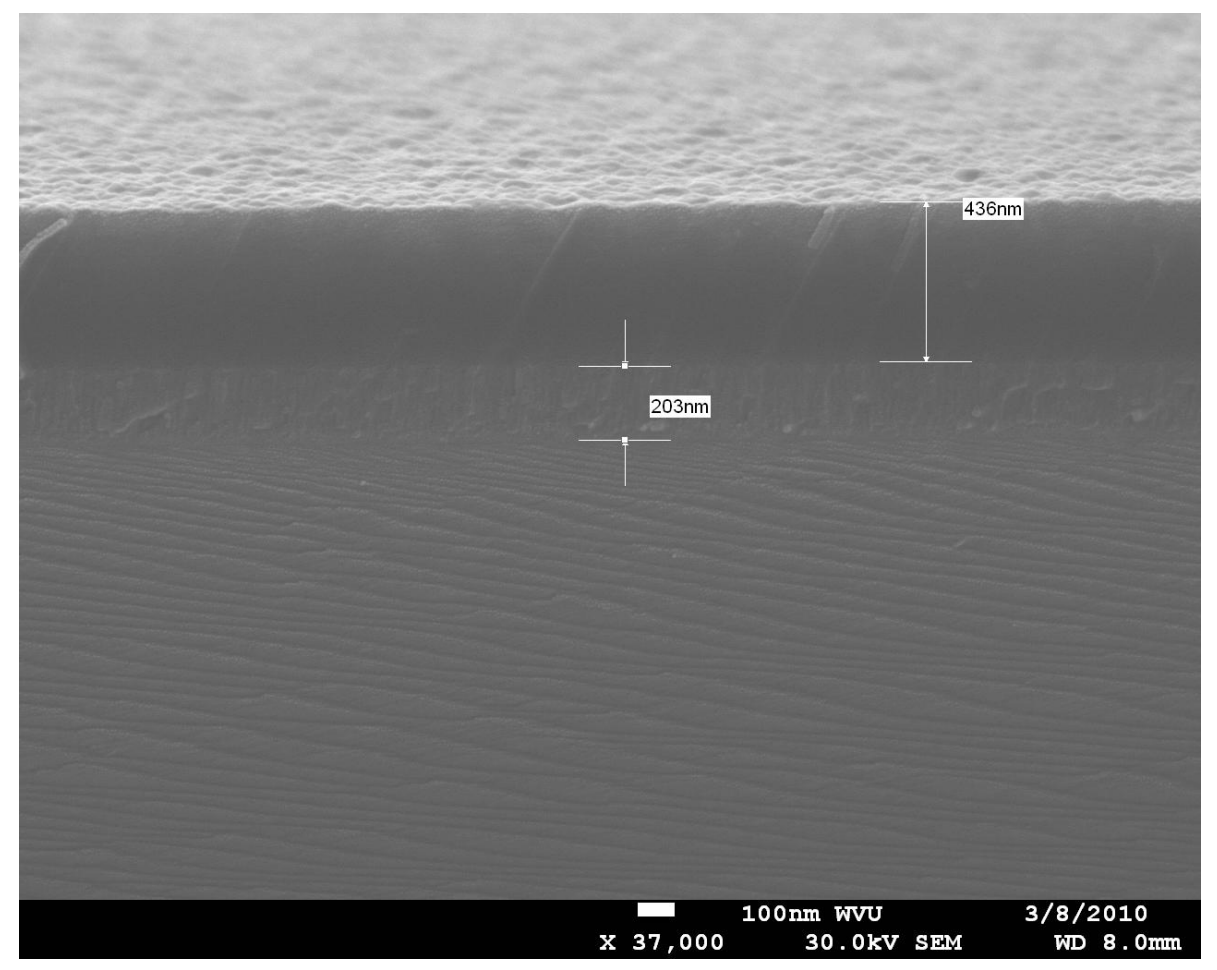

Figure 4. 5 SEM image of $\mathrm{SiO}_{2}$ deposited on Sputtered AlN

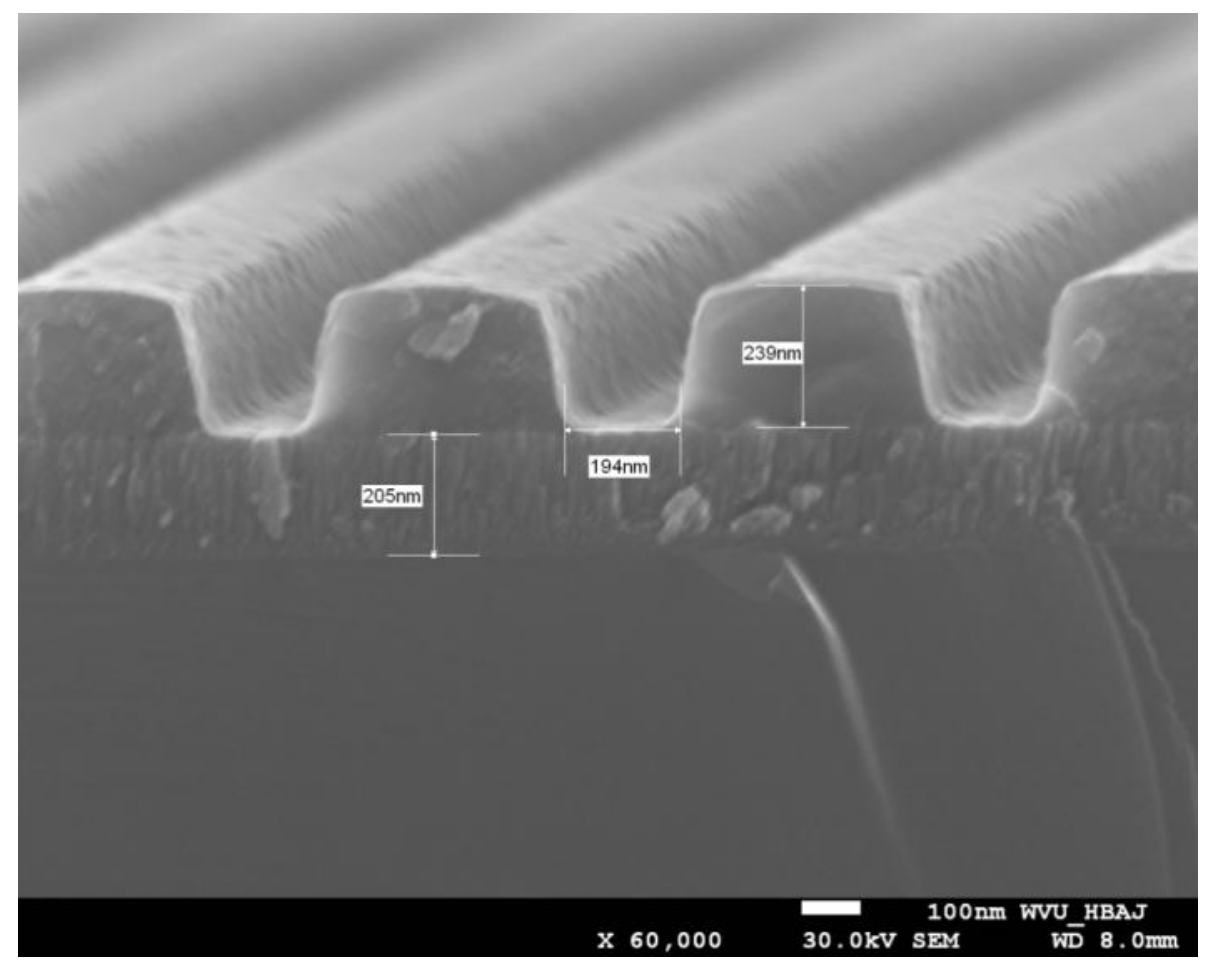

Figure 4.6 1-D PhC patterns in E-beam resist spun on AlN after development for $35 \mathrm{sec}$ 


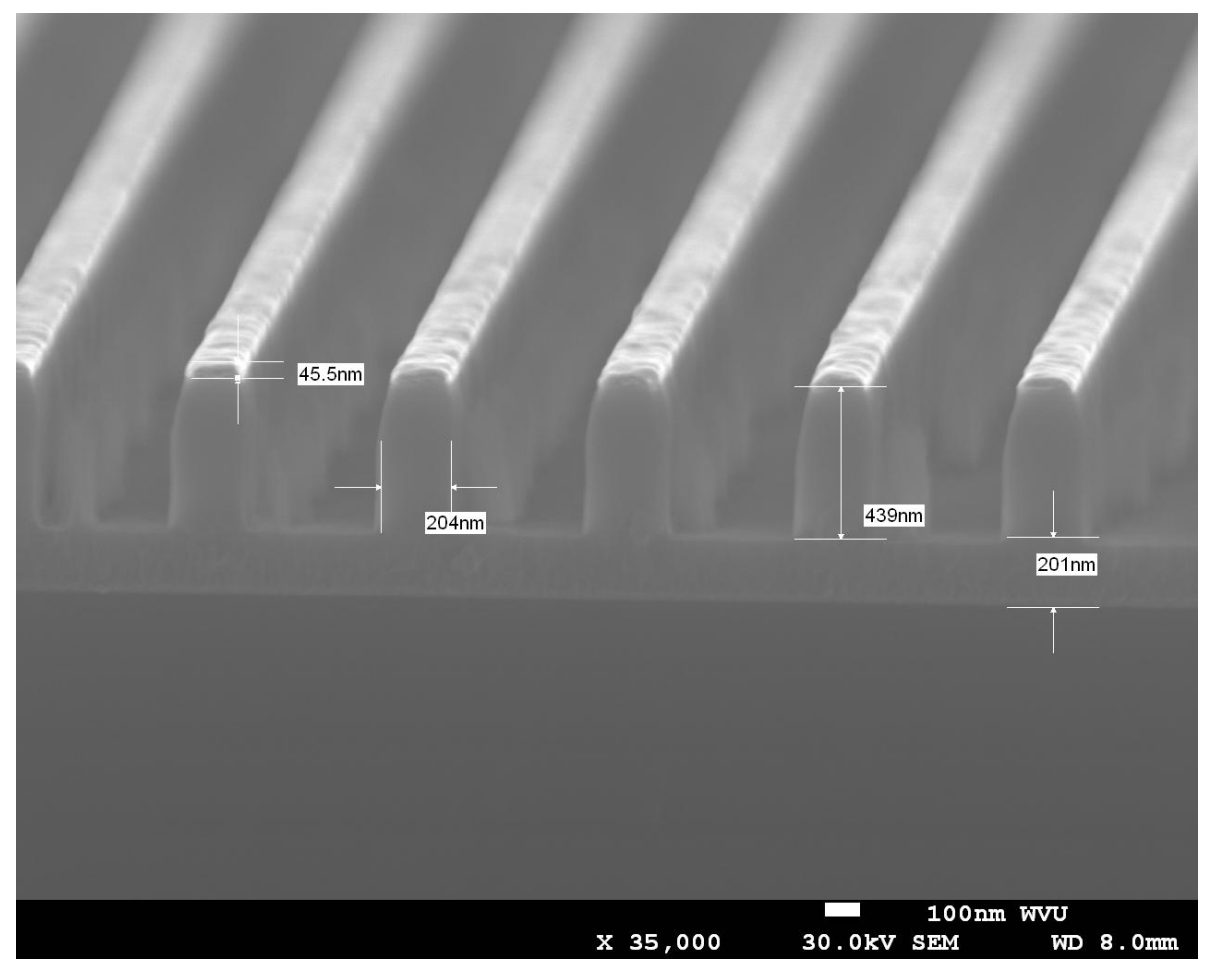

Figure 4.7 1-D PhC Patterns in $\mathrm{SiO}_{2}$ with $\mathrm{Ni}$ on top

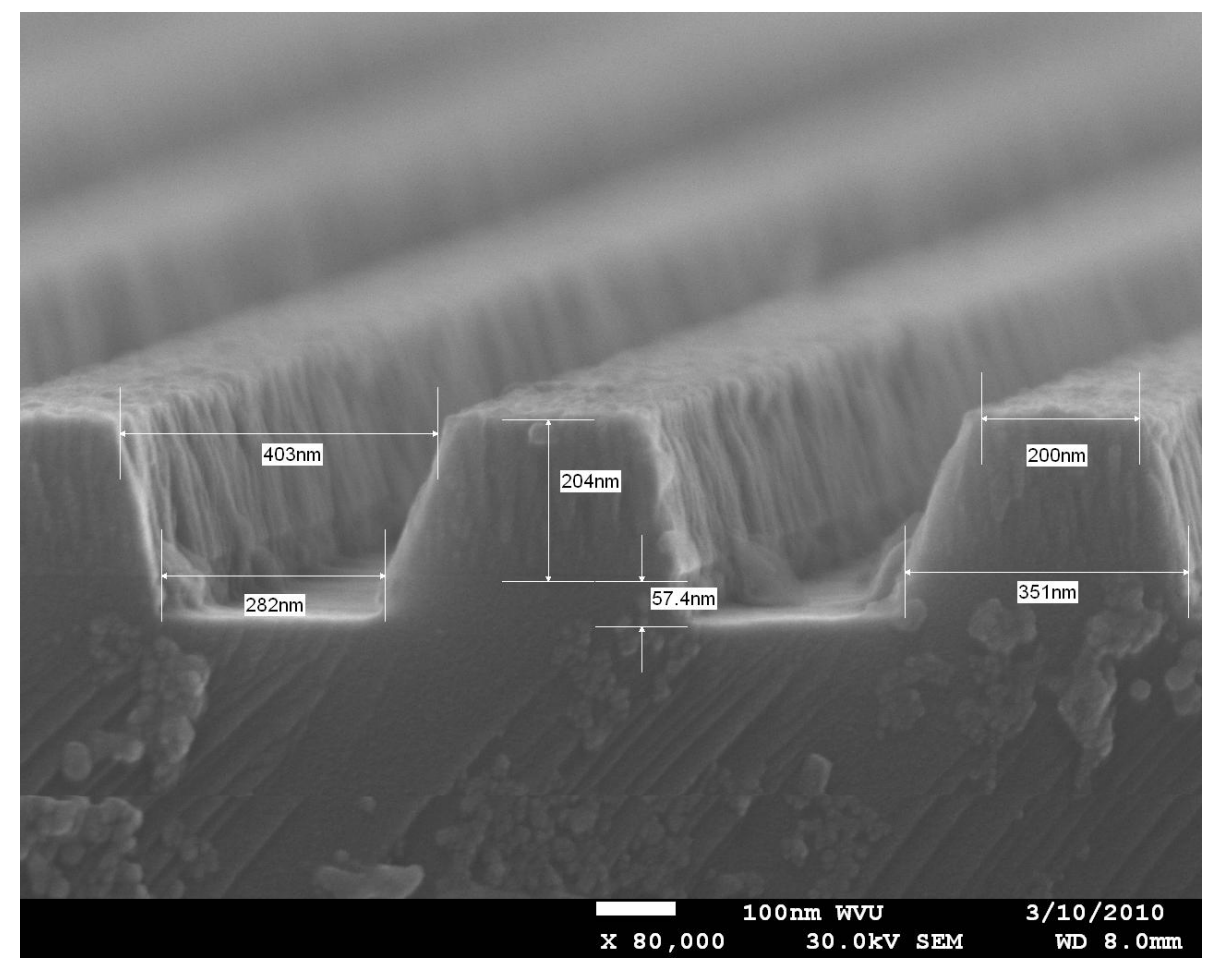

Figure 4.8 1-D PhC patterns in AlN etched into $\mathrm{Si}$ 


\subsection{Fabrication for Piezoelectric Characterization}

\subsubsection{Suspended AIN PhC pattern:}

Finite Element Modeling results showed that the degrees of freedom of a cantilever play a vital role in the piezoelectric deformation of the film. For a clamped-clamped suspended cantilever the deformation is high compared to non-suspended cantilever. The process flow for fabricating suspended 1-D PhCS in AlN is shown in Figure 4.9.

1. Degrease the sample

2. Spin AZ5214
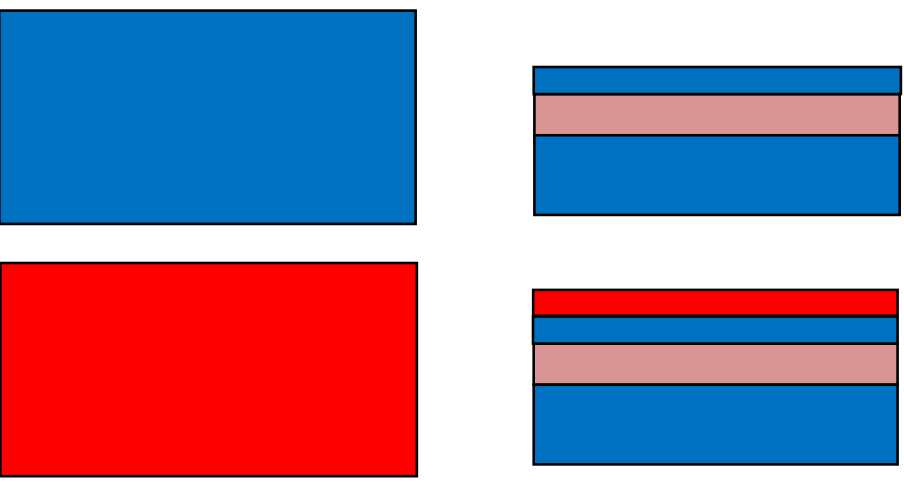

3. Patterned with photo-Lithography and Image reversal process and development.

4. Sputtered AIN ( 300nm) for metal liftoff.
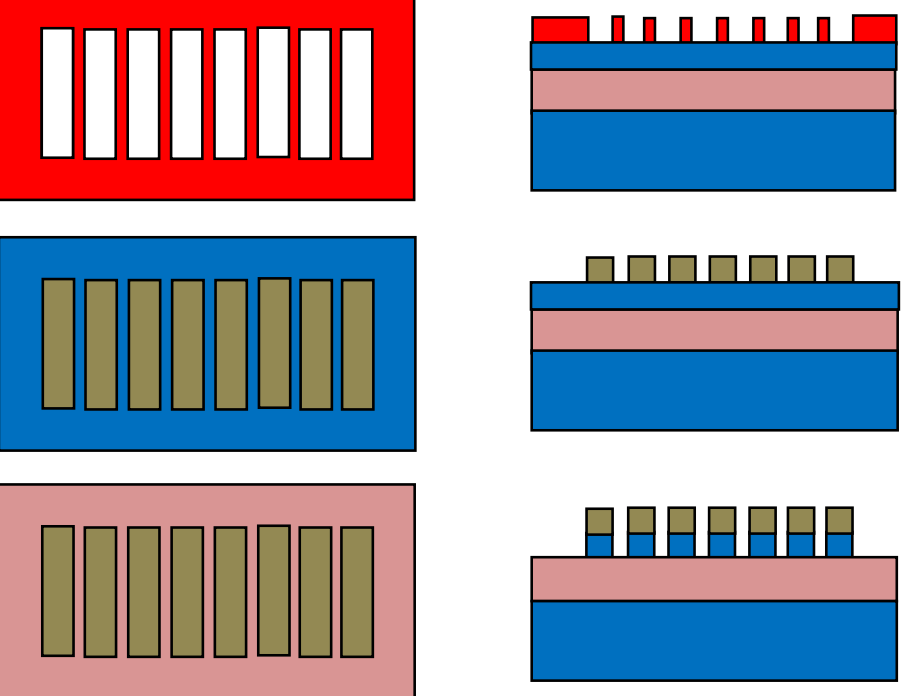

5. ICP/RIE etch for $2 \mathrm{mins}$ on $(260 / 1$
And $10 \mathrm{mins}$ for $(1.5 / 2.5 \mu \mathrm{m}) \mathrm{SOI}$
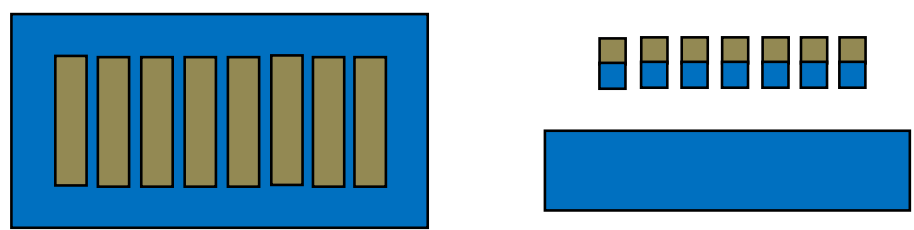

Figure 4. 9 Process flow for fabricating suspended 1-D PhCs in AlN 
Consequently, efforts were made to fabricate a suspended 1-D PhC in AlN. In this fabrication procedure SOI (Silicon on insulator) samples (with $\mathrm{Si} / \mathrm{SiO}_{2}$ dimensions $250 \mathrm{~nm} / 1 \mu \mathrm{m}$ and $1.5 / 2.5 \mu \mathrm{m}$ were used to create free standing structures instead of Si. Standard degreasing procedures were performed to clean the sample thoroughly. AZ5214 PR is spun@ @500rpm for 50sec prior to soft bake at $95^{\circ} \mathrm{C}$ for $50 \mathrm{sec}$. The samples were patterned employing photolithography technique. Image reversal bake at $120^{\circ} \mathrm{C}$ is performed for 2 min followed by flood exposure for 90sec. The samples were developed in AZ300MIF for $45 \mathrm{sec}$. Approximately, $300 \mathrm{~nm} \sim 400 \mathrm{~nm}$ AlN is sputter deposited on the samples. Acetone immersion followed by sonication is performed on the samples, which results in patterned AIN. The samples were dipped in HF to remove the underlying oxide layer of the SOI substrate, effectively suspending the lattice pattern. Even though, this procedure should result in suspended 1-D PhCs in AlN, there were certain issues that were not taken into account while fabricating suspended 1-D PhCs in AlN which resulted in failures. The problems are discussed in the issues section.

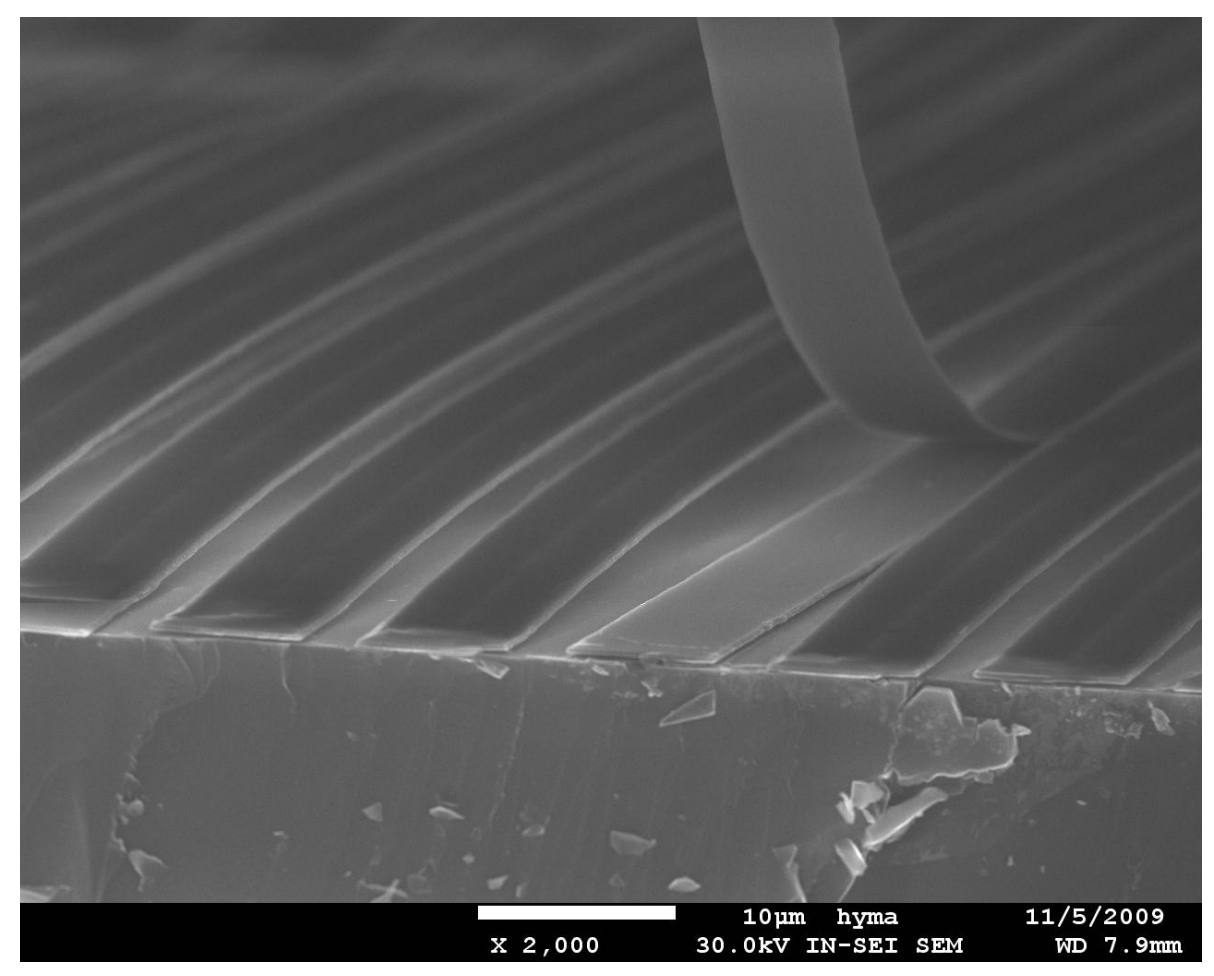

Figure 4. 10 Debonding of AIN from Si 


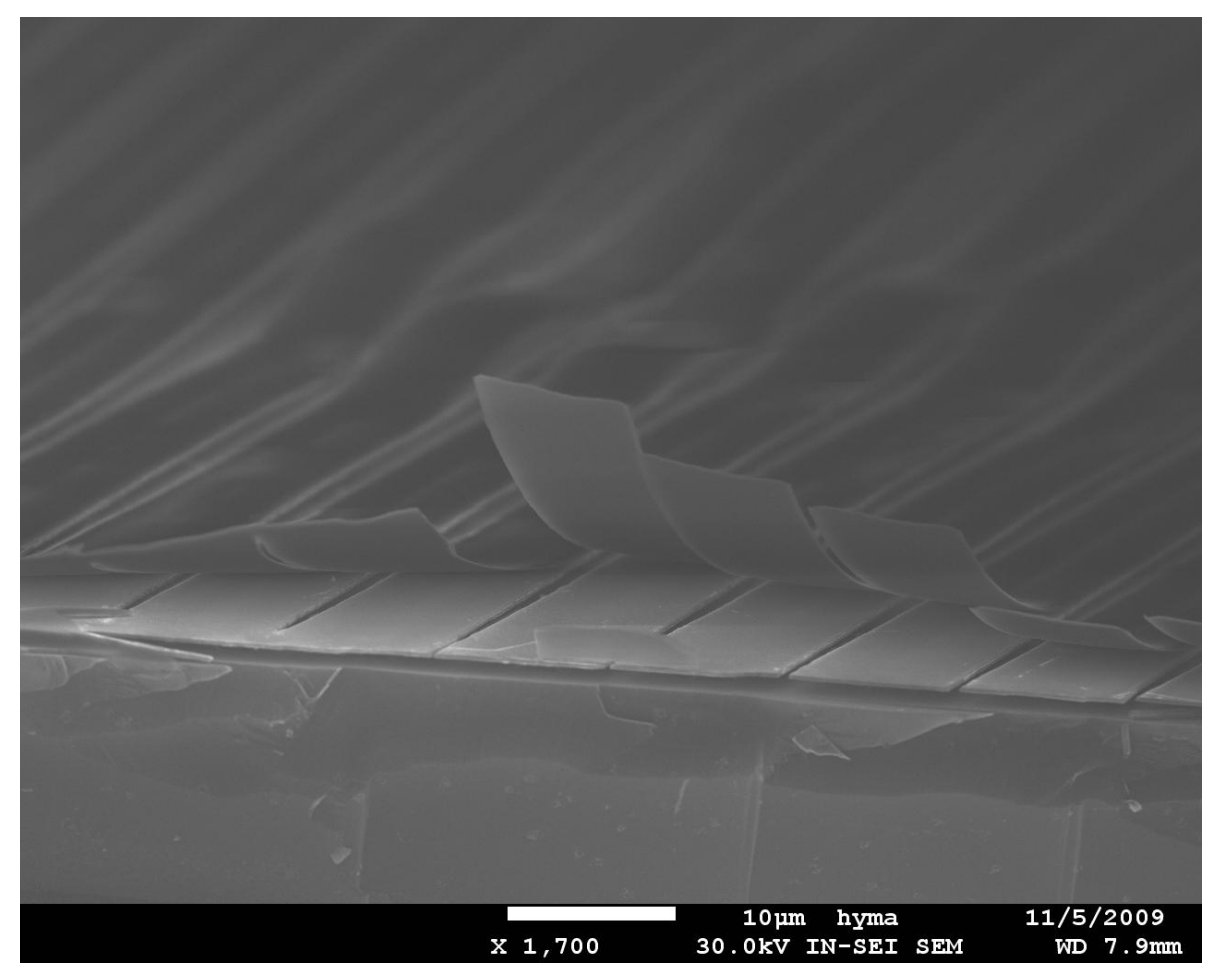

Figure 4. 11 Debonding in AIN

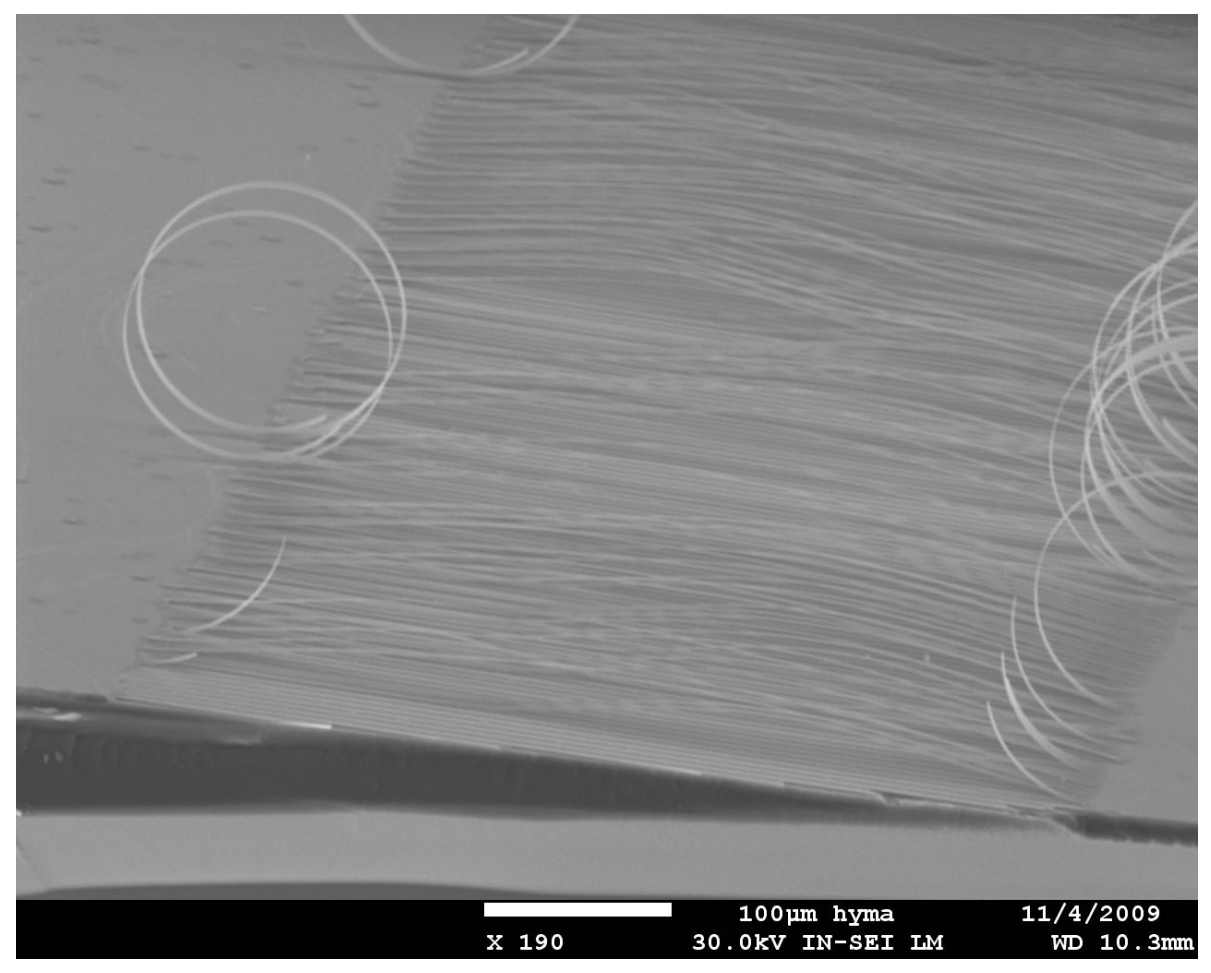

Figure 4. 12 Buckling phenomena 


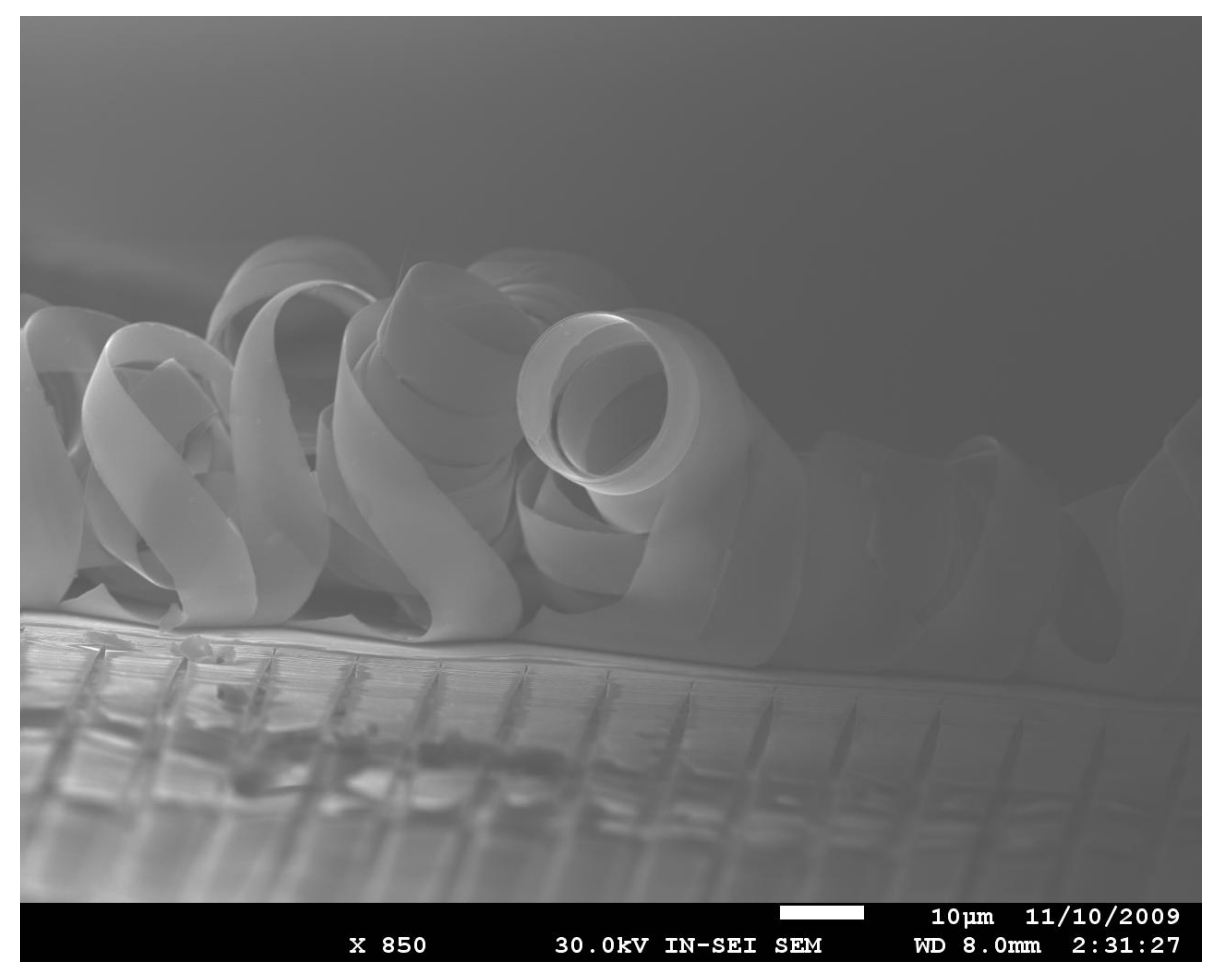

Figure 4. 13 Buckling phenomena of $\mathrm{AlN}$ in $\mathrm{Si}$

\section{ISSUE-1: Length of the pattern}

The main issue in the fabrication of surface micro machined structures is that arbitrary dimensions for the cantilever beams are not allowed due to the limitations imposed by external forces, fabrication process e.t.c. 1-D PhCs in our design is a case of clamped-clamped beams where the distributed forces arising from weight of the beam impose limitations on beam length. As the beam length is increased, these forces eventually cause the beam to touch the surface of the substrate. To understand the mechanical behavior of the clamped -clamped beams, a first step would be calculation of the spring constant of the beam. The spring constant for a clamped-clamped beam with distributed load is given by ${ }^{63}$

$$
\mathrm{k}_{\mathrm{z}}=32 \mathrm{Ew}\left(\frac{\mathrm{t}}{\mathrm{l}}\right)^{3}
$$

As already mentioned, the beam touches the substrate due to many reasons, initially collapse of the beam due to its own weight is considered. Using the mechanical equation of motion, the critical beam length can be calculated as follows

$$
\begin{gathered}
\mathrm{F}_{\mathrm{z}}=\mathrm{k}_{\mathrm{z}} \cdot \mathrm{z} \\
\mathrm{m} \cdot \mathrm{g}=\mathrm{k}_{\mathrm{z}} \cdot \mathrm{z}
\end{gathered}
$$




$$
\mathrm{m} \cdot \mathrm{g}=32 \mathrm{Ew}\left(\frac{\mathrm{t}}{\mathrm{l}}\right)^{3} \cdot \mathrm{z}
$$

where $\quad m=$ density/volume $=\rho / V$

and volume $=$ l.w.t

Therefore critical length can be derived as ${ }^{64}$

$$
l_{c r i}=\sqrt[4]{\frac{32 E z t^{2}}{\rho . g}}
$$

Critical length of a silicon beam of width $7 \mu \mathrm{m}$, thickness of $260 \mathrm{~nm}$, density $2330 \mathrm{~kg} / \mathrm{m}^{3}, 1 \mu \mathrm{m}$ spacing from the substrate and Young's modulus 150GPa can be calculated using Equation 4.4 and is found to be $1.94 \mathrm{~mm}$.

Long beams are difficult to release after wet etching of the sacrificial layer such as $\mathrm{SiO}_{2}$ using $\mathrm{HF}$. During final drying, the beams pull down to the substrate due to the surface tension from liquid trapped under the beams.

The beam length that was used earlier was nearly $4 \mathrm{~mm}$ which resulted in stiction ${ }^{65}$. To eliminate the possibility of stiction, $400 \mu \mathrm{m}$ long beams were considered for further processing.

\section{ISSUE-2: Delamination and buckling Effect}

Thin films are grown on the substrates using different techniques such as Sputtering, MOVPE e.t.c. Residual stresses may cause cracks in the film, or in the substrate, or on the film-substrate interface. These phenomena can be observed in daily life such as peeling of paints and pavements cracking ${ }^{66}$. Using the above discussed deposition techniques, the films are grown at a fixed temperature. After the film is grown, the temperature is changed to a different level. Due to this variation in the temperature, the substrate acquires a thermal strain but remains stressfree; also the film acquires a thermal strain which is different from the substrate. This difference can be related to the difference in the thermal expansion coefficients of the substrate and the thin film. When the film and the substrate are well-bonded, the net in-plane strain must be the same as thermal strain of substrate. As a result, the mismatch strain has to be accommodated by the thin film, either by elastic or inelastic deformation. A film under tensile stress may debond from the edge of the film or from the root of a channel crack. When the debond length exceeds several times the film thickness, the debonding process attains a steady-state, and the driving force becomes independent of the debond length ${ }^{11}$. 


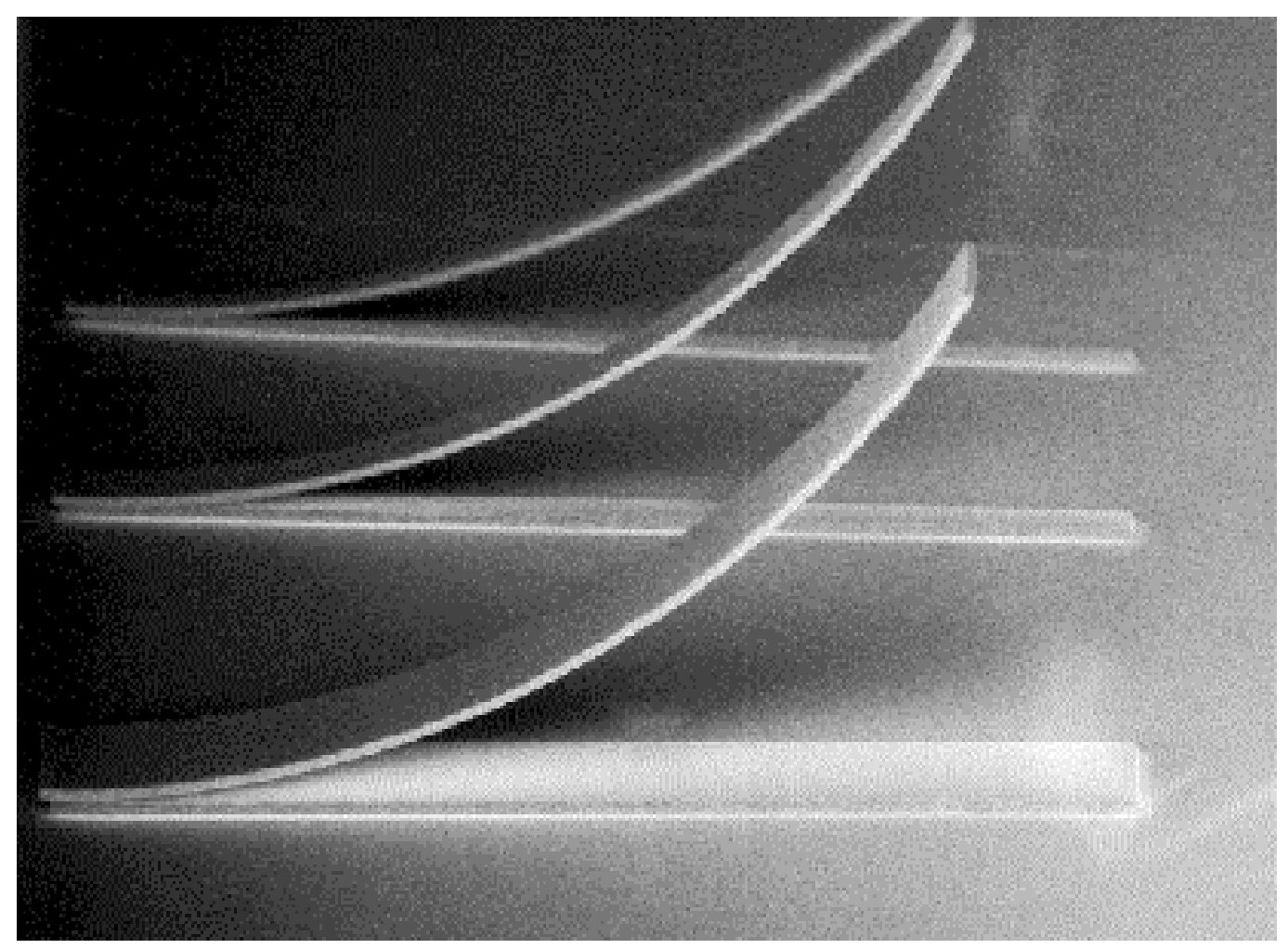

Figure 4. 14 Debond from an edge of the film, driven by residual tensile stress ${ }^{11}$ (Courtesy of Q. Ma of Intel Corp)

From figure 4.3, when the film that was deboned approaches the other edge of the film, the debond driving force decreases, so that the debonding stops before it reaches the film edge ${ }^{11}$. If the film is under compressive stress, then a certain area underneath it is unbounded to substrate which might result in buckling of the film. The AlN films that were released from the Si substrate exhibited the debonding phenomena due to tensile stress as shown in Figures 4.5 4.6. Buckling phenomena was exhibited due to compressive stress when the thin film comprised of both AlN and $\mathrm{Ti} / \mathrm{Pt}$ as shown in Figure 4.7 and Figure 4.8. Even though, the sputtering pressure was varied ${ }^{67}$ to reduce both compressive and tensile stress in the films; stress prevailed in the films.

\subsubsection{Clamped 1-D PhCs in AlN}

The tensile and compressive stress prevailed significantly in the AlN thin films even after various measures were taken to reduce the same during the process of sputtering. The process flow for fabricating clamped 1-D PhCs in AlN is shown in Figure 4.15. 
1. Degrease the sample
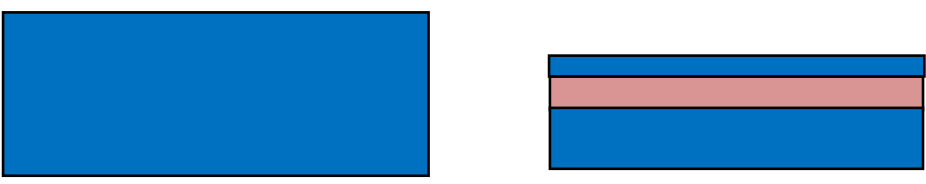

2. Spin AZ5214

3. Patterned with optical-Lithography
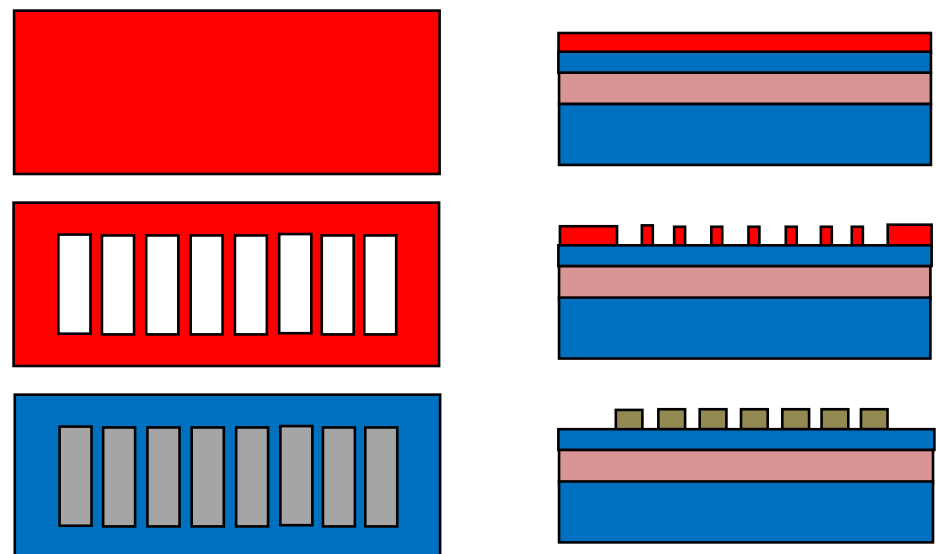

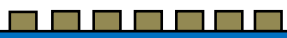

4. Sputtered $\operatorname{Cr}(\sim 300 \mathrm{~nm})$ for metal liftoff

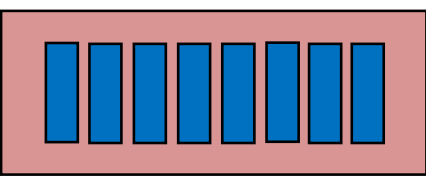

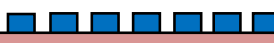

5. ICP/RIE etch for $2 \mathrm{mins}$ on $(260 / 1$ $\mu \mathrm{m}) \mathrm{SOI}$ And $10 \mathrm{mins}$ for $(1.5 / 2.5 \mu \mathrm{m}) \mathrm{SOI}$

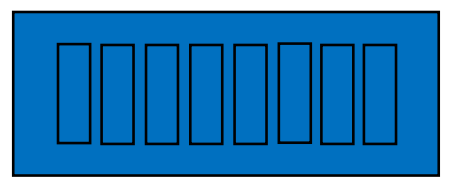

$\square \square \square \square \square \square \square$

6. Agitate gently in HF to remove $\mathrm{SiO} 2$ (4min-8mins)
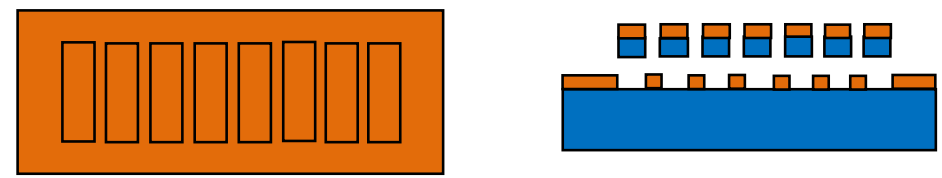
structures
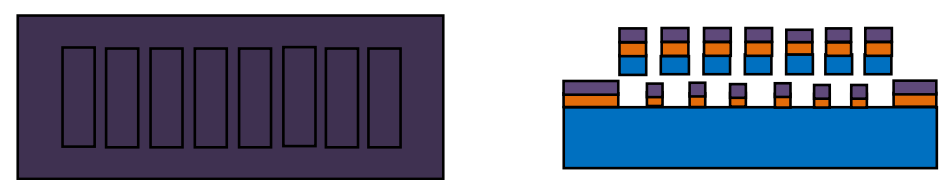

Figure 4. 15 Process Flow for fabrication of Clamped 1-D PhCs

Consequently, clamped 1-D PhCs were realized for measuring the piezoelectric coefficient $\mathrm{d}_{33}$. In this process, $\mathrm{Si}$ samples were degreased and were patterned using the negative lithography technique discussed earlier. The patterns were transferred into $\mathrm{Si}$ using $\mathrm{Ni}$ as etch mask in ICP/RIE etching technique. The samples were eventually dipped in HF to release the clamped-clamped beams. AlN is sputtered at $45 \mathrm{mTorr}$ pressure with $\mathrm{Ar} / \mathrm{N}_{2}$ gas concentration of $3 / 27$ after a presputter of $\mathrm{Al}$ at $60 \mathrm{mTorr}$ pressure and $\mathrm{Ar}$ gas concentration of $30 \mathrm{sccm}$ for 10 minutes to remove impurities. Later, Ti/Pt was sputtered on the sample to act as a top electrode. The SEM images are shown below. 
After the deposition of $\mathrm{Ti} / \mathrm{Pt}$, when the samples were broken to view the cross section, due to the pressure on the samples, the surfaces appeared to be pinned to the substrate.

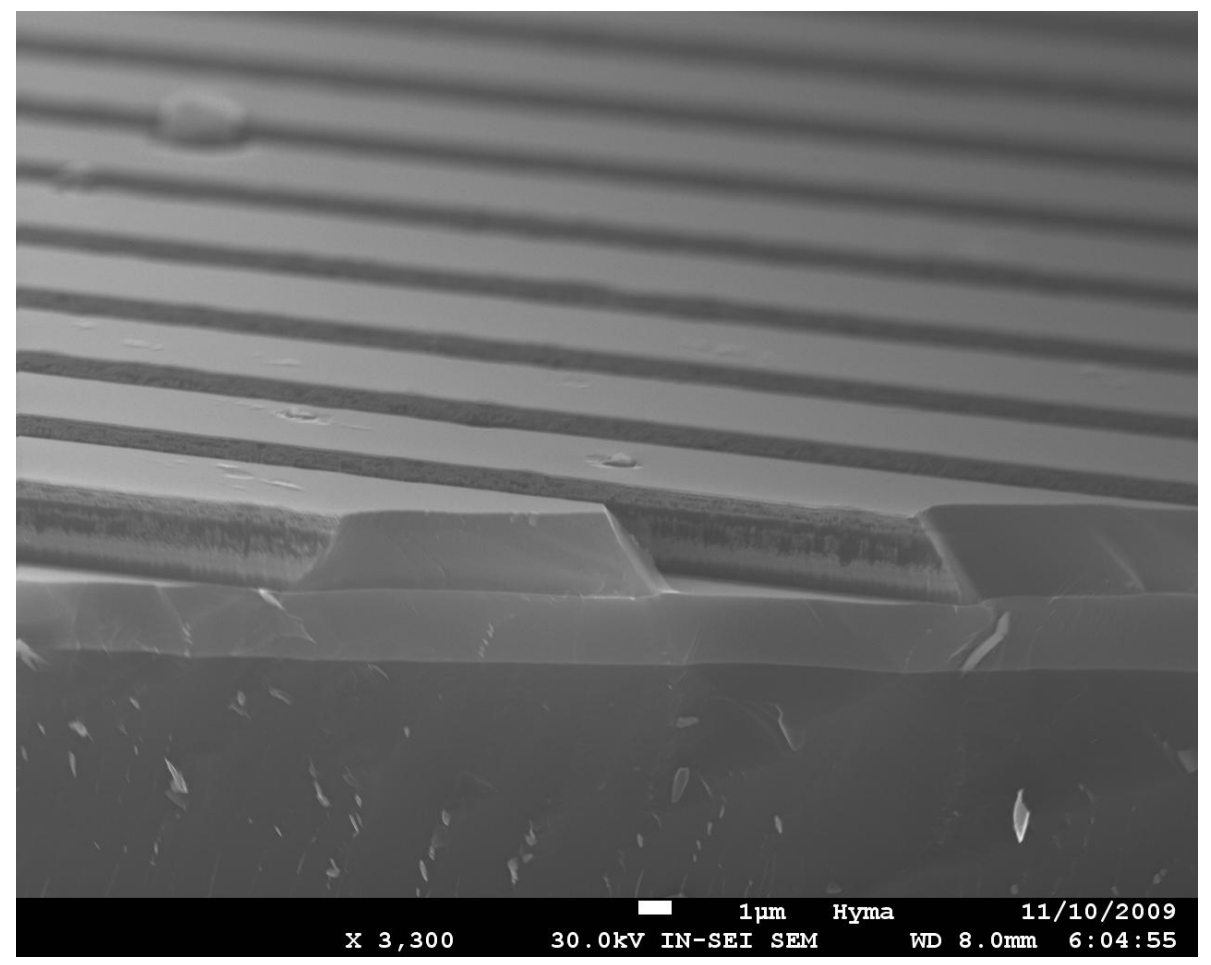

Figure 4. 16 SOI samples with 1-D PhCs transferred into $\mathrm{Si}\left(1.5 \mu \mathrm{mSi} / 2.5 \mu \mathrm{mSiO}_{2}\right)$ after ICP/RIE etch

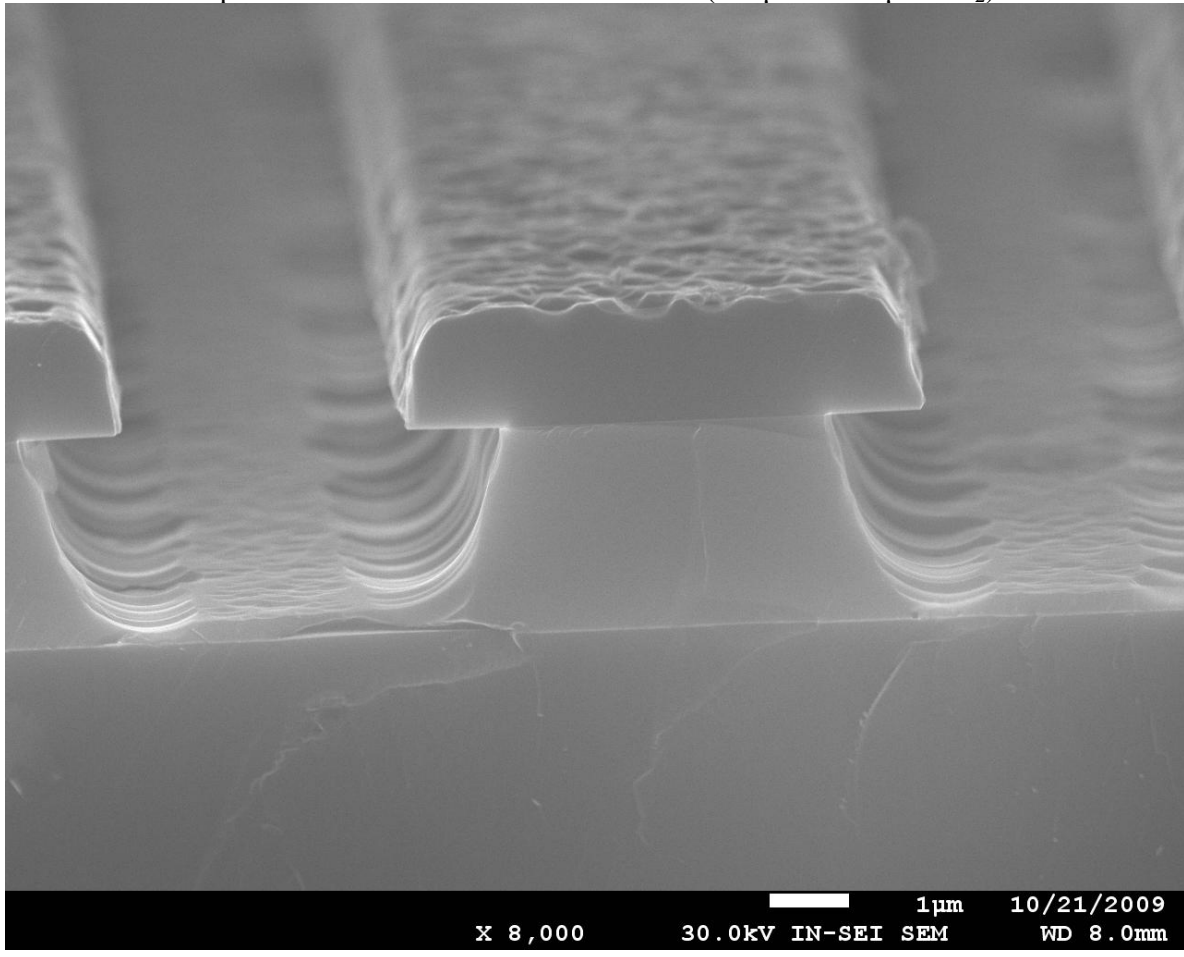

Figure 4. 3 1-D PhC pattern after 1 min etch of $\mathrm{SiO}_{2}$ in $\mathrm{HF}$ 


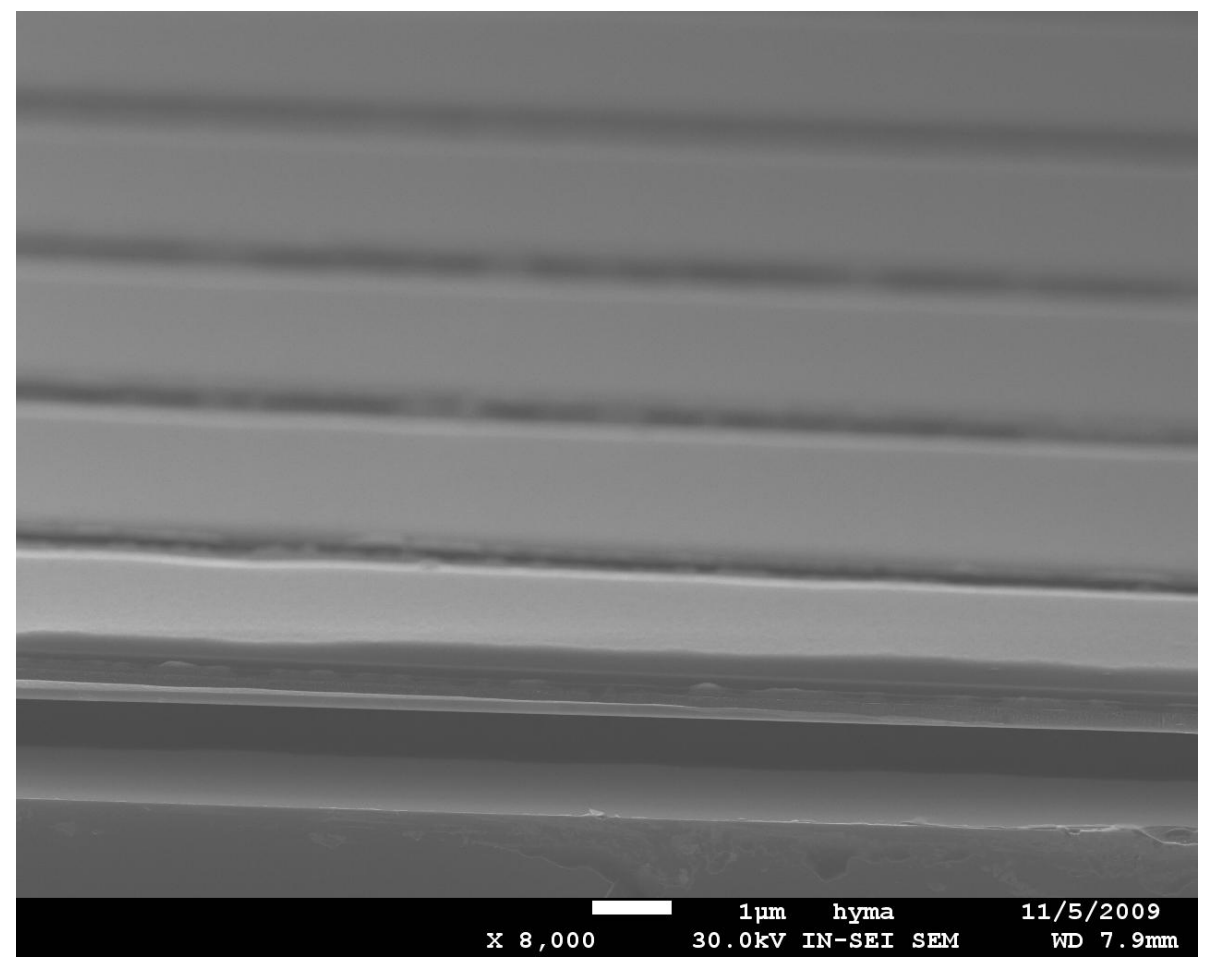

Figure 4. 4 Side view of 1-D PhC patterns in Si suspended or released from substrate

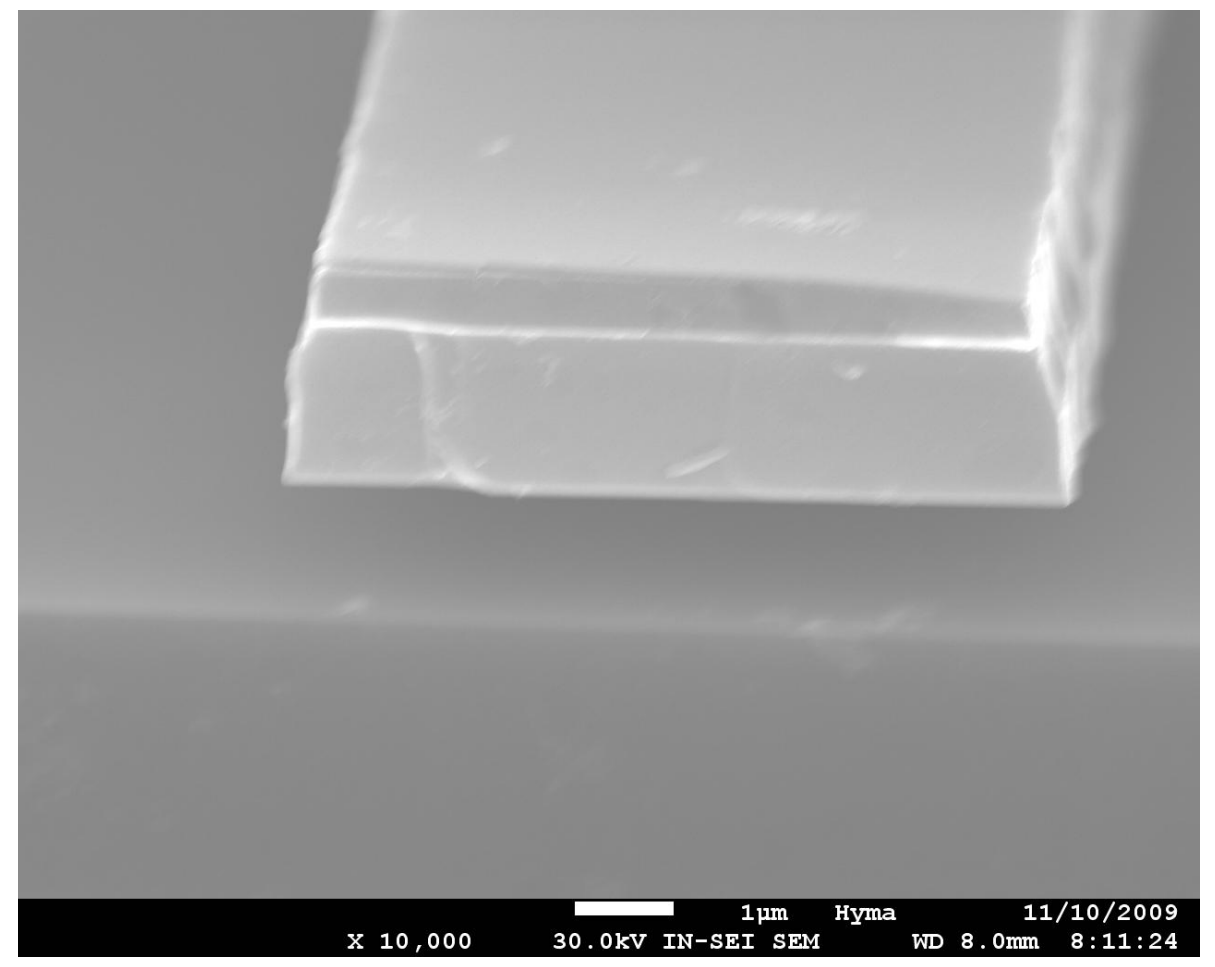

Figure 4. 5 Suspended 1-D PhCs in $\mathrm{Si}$ after $\mathrm{SiO}_{2}$ etch in $\mathrm{HF}$ 


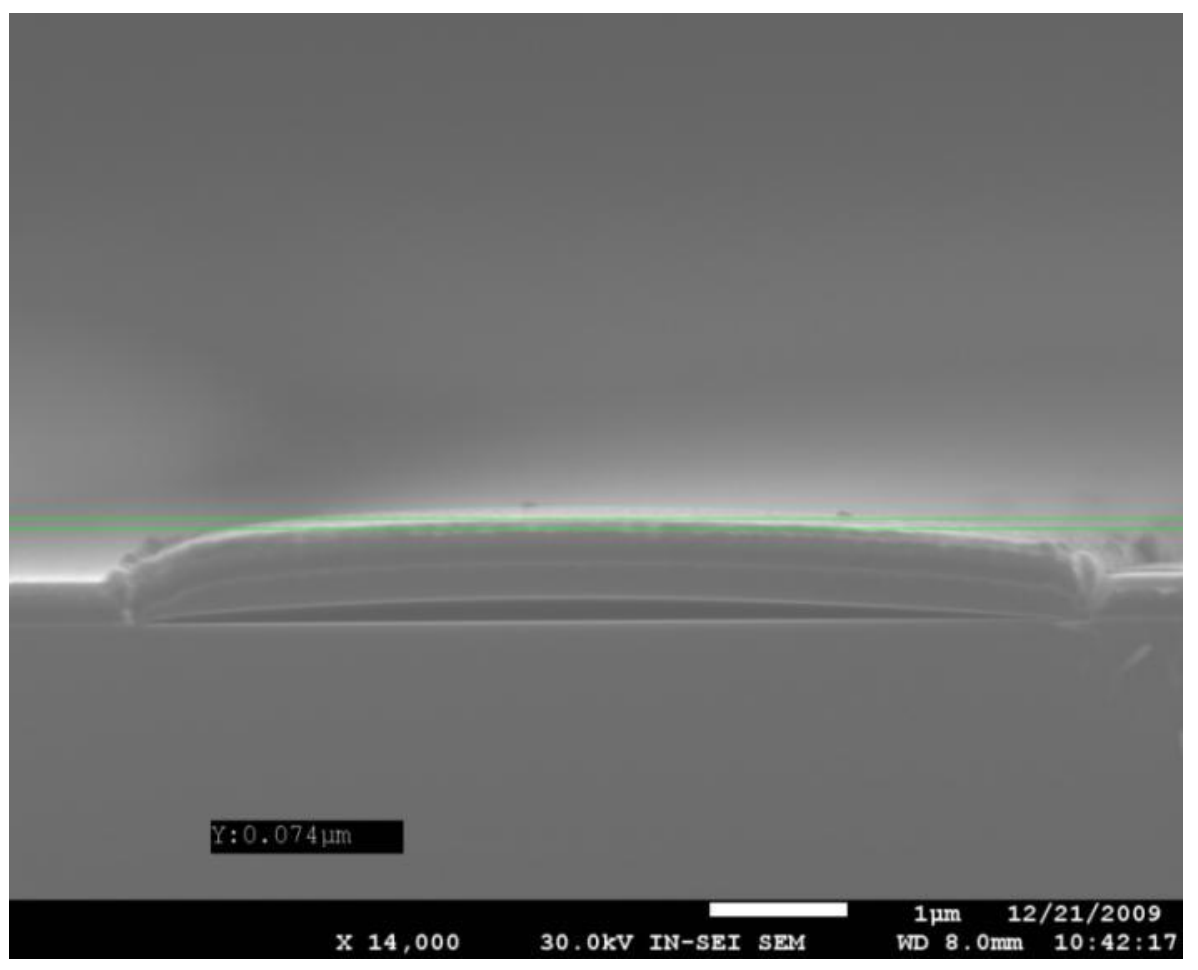

Figure 4. 20 Clamped 1-D PhCs after AlN deposition on suspended Si PhC patterns followed by Pt-image 01

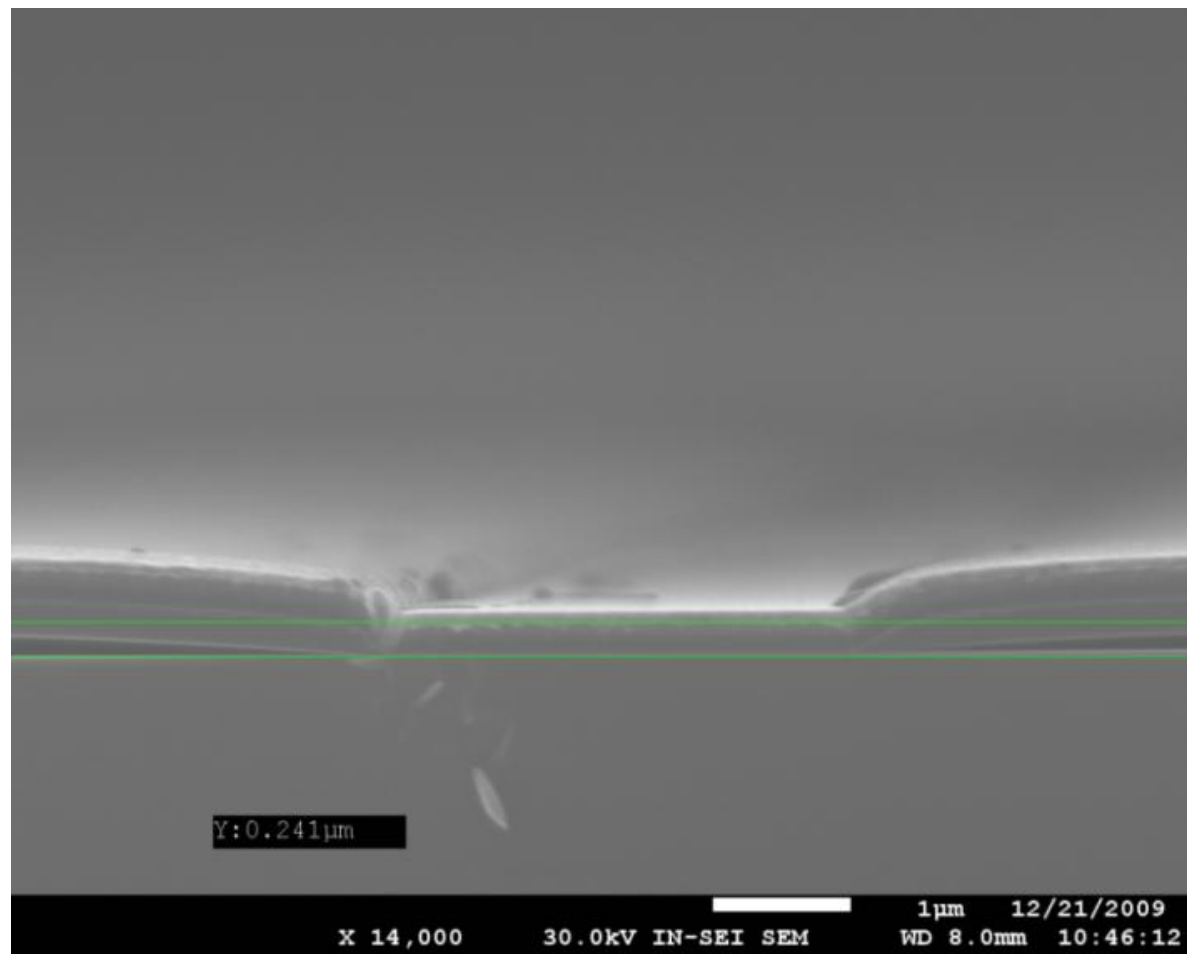

Figure 4. 21 Clamped 1-D PhCs after AIN deposition on suspended Si PhC patterns followed by Pt- Image02 


\subsection{SUMMARY}

Nanometer scale 1-D PhCs were successfully fabricated in Si substrate and AlN thin films which can be used for optical characterization. Optimization in the process of etching of AIN is required to achieve perfect sidewalls in nanometer scale patterns. Fabrication of suspended micron-scale 1-D PhCs in AlN resulted in buckling effect which might be accounted for the stress problems due to sputtered AlN. Process for sputtering AIN needs further optimization in order to create suspended AIN 1-D PhCs on SOI substrates.
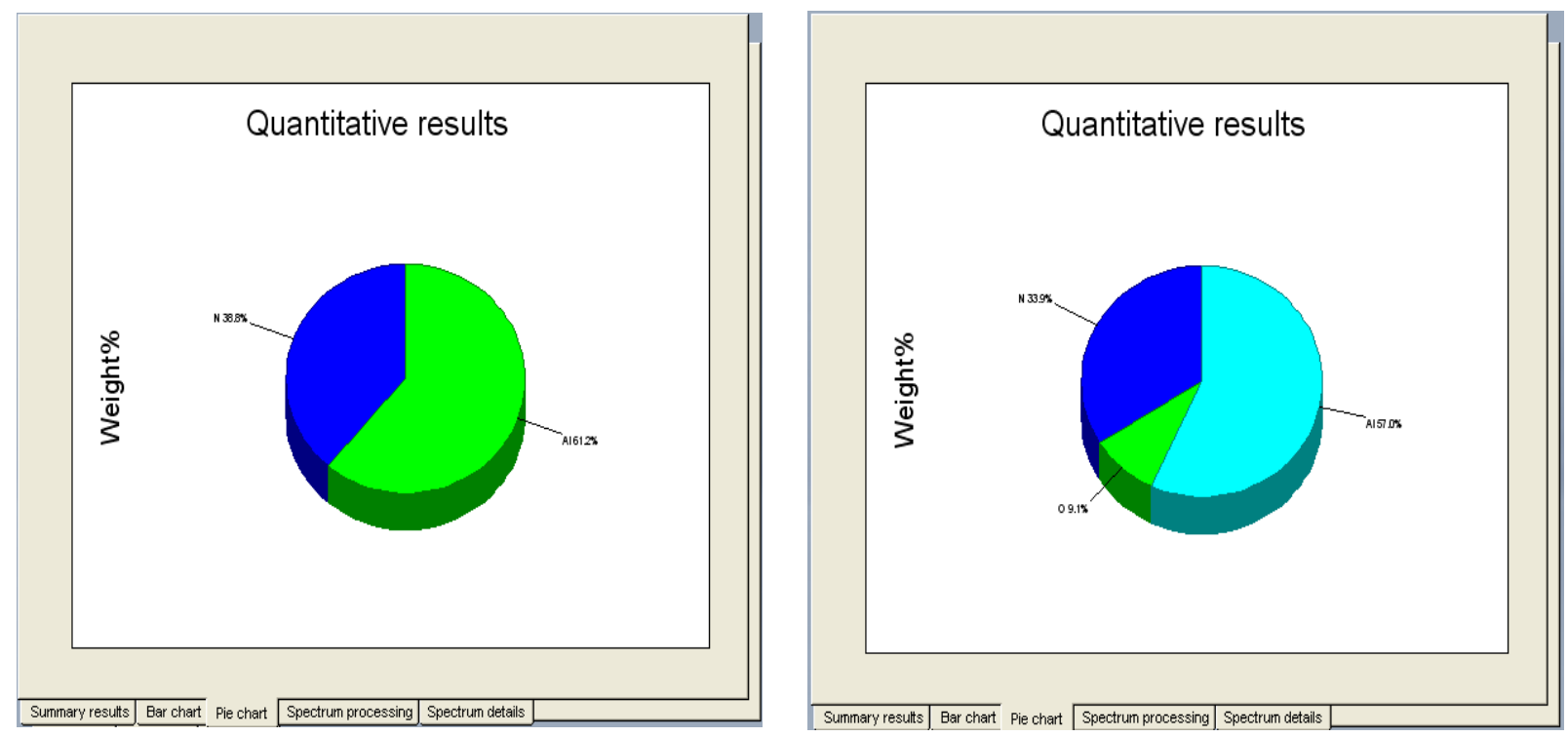

Figure 4.22 (a\&b) Composition of Al \& $\mathrm{N}_{2}$ in Sputtered AIN on Si and SOI wafers respectively

EDAX measurements provide a general idea of atomic compositions of elements in a compound. EDAX measurements were performed on AlN sputtered on Si and SOI to analyze the atomic compositions as shown in Figure 4.22. But before drawing any conclusion from these results, it is recommended to compare the results with the results drawn from MOCVD grown AIN using an accurate measuring technique. 


\section{CHAPTER 5: CHARACTERIZATION}

\subsection{Optical Characterization}

The optical bandgap of planar PhCs reveals their unique properties such as transmittance, reflectivity, diffraction efficiency, losses, absorption, and reduced group velocity e.t.c. These properties can be used to implement both linear and non-linear devices, such as photonic crystal fibers, filters, biosensors e.t.c. 1-D PhCs that were designed and fabricated in this effort were tested to determine transmission and reflectivity properties of the PhC lattice. The techniques for experimentally determining and plotting band diagrams, as well as waveguide characterization using prism coupler, will be discussed in this chapter.

\subsection{Photonic Crystal Characterization}

\subsubsection{Band Diagram Characterization Techniques using Normal Incidence}

For a periodic optical wave with an angle of incidence normal to the PhC lattice; the wave undergoes several optical phenomena which result in the formation of a band gap. Band gap comparison of the as-designed and fabricated $\mathrm{PhCs}$ is very important because variation in device geometries during the fabrication phase may alter the device functionality. The direct measurement of optical spectra below the light cone can be attained using various optical setups. Coupling of light into the thin films to subsequently reach nanometer scale $\mathrm{PhC}$ features in order to measure bandgap is challenging. Some of the coupling techniques are discussed below.

\section{(i)End-fire coupling technique:}

Optical setup for implementing this technique typically consists of a light source, Light-emitting diode(LED), polarizers as $\mathrm{PhCs}$ are polarization sensitive, optical fibers which can be used as connectors for input and output, IR camera, detector, spectrometer e.t.c as shown in the Figure5.2.

End-fire characterization setups using a on a multi-port channel drop filter ${ }^{68}$ and using Mach-Zehnder interferometer $^{69}$ are some good examples which are used for complex or advanced device designs. Ideally, this technique gives a direct measurement of optical properties of a $\mathrm{PhC}$ device. But practically the output signal obtained from such a setup is degraded due to some interference fringes caused by the parasitic reflections from sample facets, tapers e.t.c. Optimization of fabrication steps may lower the degradation caused by reflections to an extent however it does not completely circumvent them. 


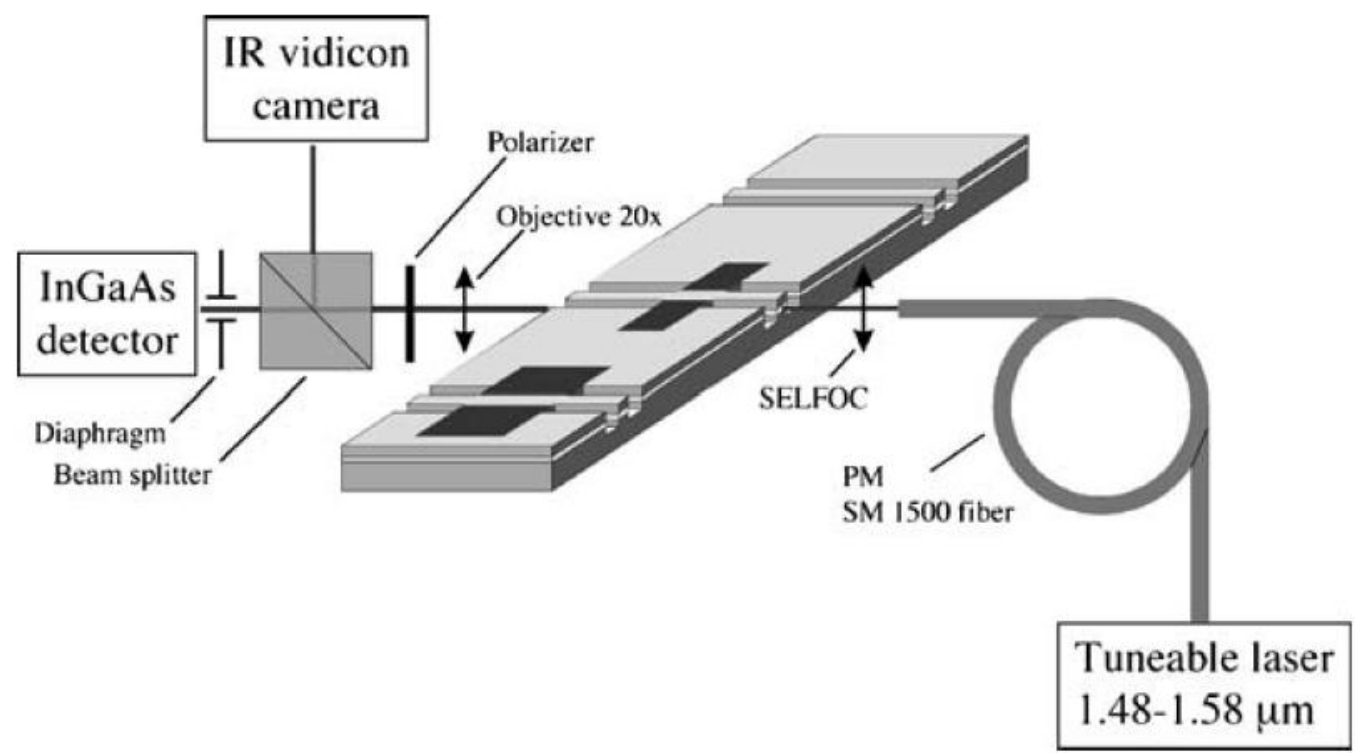

Figure 5.2Schematic of an end-fire measurement ${ }^{70}$.

\section{(ii) Internal Light Source (ILS) Technique:}

ILS technique is mainly designed for III-V planar PhCs, and h does not require complete fabrication of waveguide patterns for light propagation into the sample. Instead of coupling light into the sample at the end facets, light emitters which are potentially either quantum wells (QW) or quantum dots (QD) are inserted into the planar waveguides wherever needed. The photoluminescence (PL) of the light emitters is excited and the beam coupled travels as a guided mode towards PhC lattice. The image of the guided beam emitted from the sample through a cleaved facet is coupled into a spectrometer for spectral analysis. The signal obtained at the cleaved facet may contain a beam that propagated through air, planar waveguide and substrate which will result in cross-talk. This cross-talk can be avoided by using large edges and keeping the excitation spot far away from cleaved facet. The schematic experimental setup is as shown in Figure 5.3 
(a)

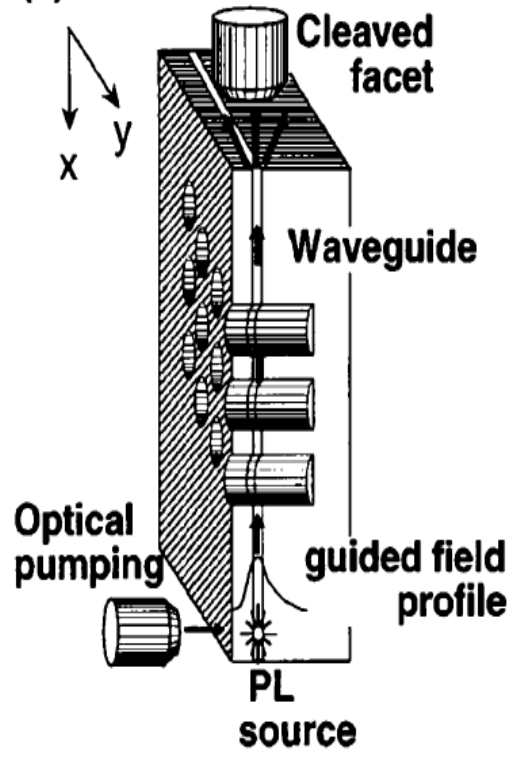

(b)

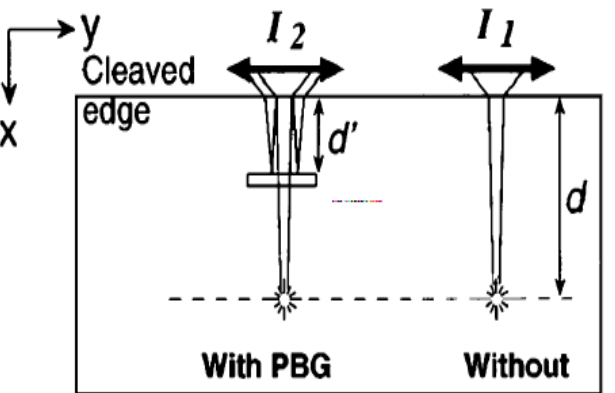

(c)

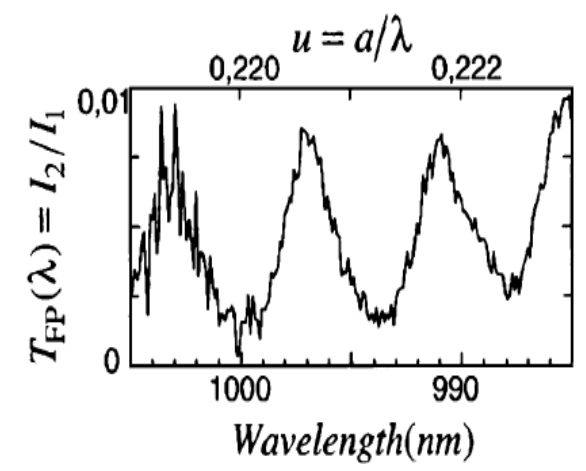

Figure 5.3(a) Schematic of ILS experiment (b) side-view of schematic (c) Typical experimental Transmission ${ }^{71}$

In the Figure 5.3(b) the reference signal $I_{1}$ and the signal from PhC lattice $I_{2}$ are measured by keeping the distance from the excitation point to the cleaved facet constant. The ration of $I_{2} / I_{1}$ yields the absolute transmission spectrum as shown in Figure 5.3(c). The main disadvantage of this technique is that absorption from light emitters layer result in propagation losses and reduced quality factors for high performance structures. These are some of the most frequently used techniques for bandgap characterization. Some other advanced techniques like Local probe Scanning near-field optical Microscopy $(\mathrm{SNOM})^{1}$, Fourier imaging ${ }^{1}$ e.t.c are used less often because of their complexity.

\subsubsection{Band diagram Characterization using Surface Coupling Technique}

The techniques described in section 5.1.1 can be used to determine the band diagram of a photonic crystal slab below the light cone. However, the bands above light cone are not easy to determine using ILS or end-fire coupling techniques. Even though the determination of bands above the light cone is not so significant for most of the PhC based devices such as biosensors; strong dispersive effects above the light cone show a significant promise to solve the long-standing problem of light extraction from light emitting diodes (LEDs) ${ }^{5}$. 


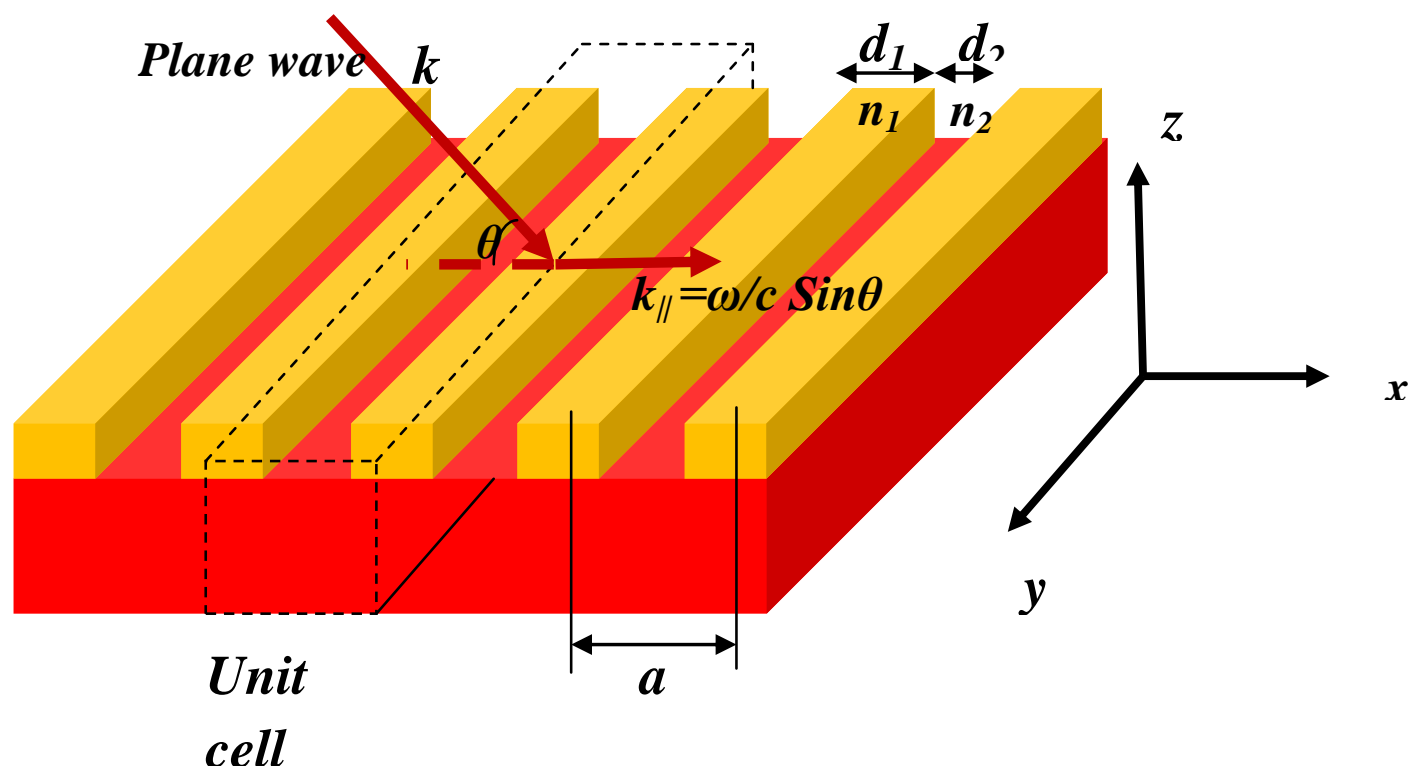

Figure 5.6 Off-axis propagation in 1-D slab $\mathrm{PhC}$

Dr. Vasily N. Astratov developed a method known as surface coupling technique ${ }^{72,73,74}$ to directly draw the dispersion curves above the light cone in PhCs. The main advantage of this technique compared to external coupling techniques is that it possesses external angular control of the group velocity and group velocity dispersion of the modes excited. In this technique, the dispersion of the leaky modes of 1-D or 2-D PhCs above light cone can be reconstructed based on resonant coupling in reflectivity as a function of wavelength and incidence angle as is shown in Figure 5.6. For a given wavelength (i.e., photon energy), resonant coupling occurs when the in plane component of the incident wave vector matches the wave vector of a corresponding photonic mode. If the light reflected off the sample surface is measured as a function of wavelength, such coupling can then be identified as a resonance feature (e.g. a peak or dip) in the reflected spectrum. By recording such spectra for a number of angles of incidence, thereby varying the in-plane component of the incident wave vector, particular points of the photonic band structure can be probed, and the dispersion curves of leaky modes can be mapped to the band structure. A better understanding of dispersion curves can be gained by considering the theory of diffraction gratings optics, as discussed in Appendix B. 


\subsubsection{Earlier Work in Surfacing coupling Technique}
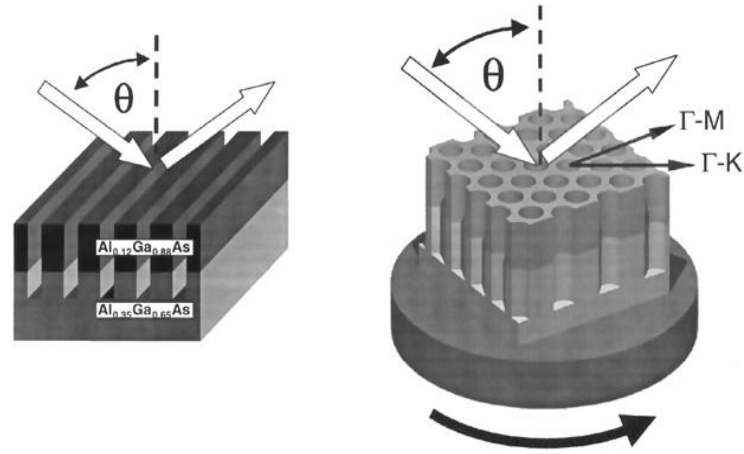

Rotation Stage

Figure 5.7 Experimental geometry of 1-D and 2-D structures used by Astratov et.al

Vasily N. Astratov et.al used the surface coupling technique to study the photonic crystal waveguides that were fabricated in the AlGaAs (Aluminum Gallium Arsenide) material system. The regular lattices of air stripes or holes are etched all the way through the surface waveguide deep into the cladding layer. Optical studies were performed with broad band plane polarized light obtained from a tungsten-halogen lamp. CCD detection was employed and the light was incident perpendicular to the stripes of the lattice at a range of angles of incidence. The specularly reflected beam was imaged to study reflectivity properties as shown in Figure 5.8 (a) of individual lattices and projected on to the slits of a grating spectrometer.

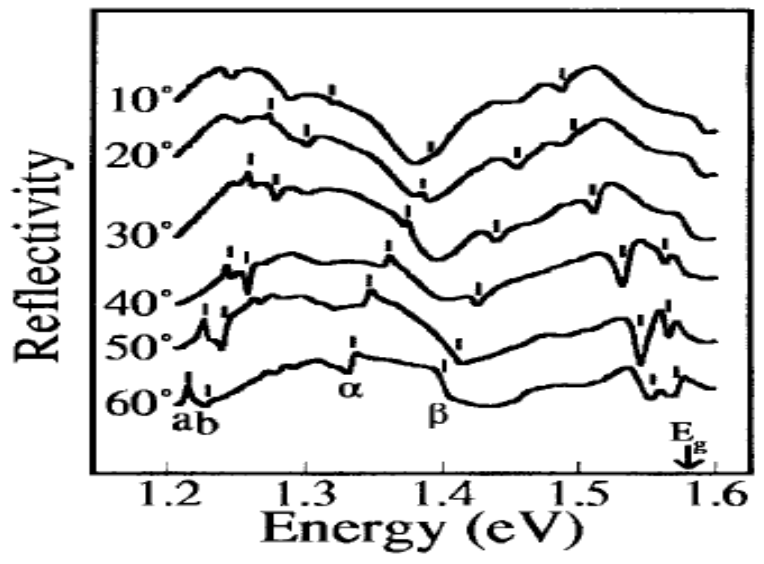

Figure 5.8 Angular dependent TM polarized reflectivity spectra from a 2-D honeycomb lattice of air cylinders with $\mathrm{a}=360 \mathrm{~nm}^{6}$.

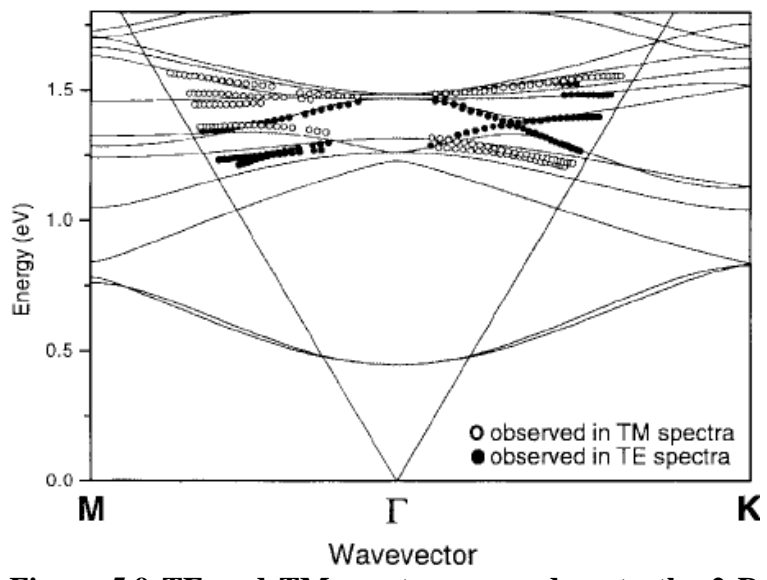

Figure 5.9 TE and TM spectra mapped on to the 2-D model of perfectly confining waveguide model with $\mathrm{PhC}$ pattern $^{6}$

Results indicated that waveguide modes in such lattices experience very high in-plane modulation of refractive index, and significant forbidden zones open up in the photonic band structure allowing, for example, important 
applications such as enhanced light extraction efficiency from solid state emitters and control of spontaneous emission. M.Patrini et.al ${ }^{75}$ characterized two different 1-D structures with different lattice constants and air-fractions using Fourier-transform spectrometer (Bruker IFS66) at a spectral resolution of $1 \mathrm{meV}$ and measured variable angle specular reflectance in the spectral range $0.25-2 \mathrm{eV}$. The plane of incidence is perpendicular to the sample surface and the angle of incidence of the collimated beam from the spectrometer varies over the range $5^{0}-75^{\circ}$. A liquidnitrogen-cooled InSb photodiode is used as the detector and a silver mirror is the absolute reflectance reference. Measurements were done for light incident along the $\Gamma-\mathrm{M}$ orientation of the 1-D crystal (i.e. perpendicular to the stripes) both in transverse electric (TE) and transverse magnetic (TM) polarizations by means of a calcite GlanTaylor polarizer. Superimposed on the interference background caused by the effective refractive index of the photonic crystal, several sharp features are observed which display a well-defined dispersion in their energy positions with increasing angle of incidence. While sample with higher air fraction displays rather weak and broad features which imply a larger coupling of folded guided modes above the light cone to leaky waveguide modes and therefore an increase of the diffraction losses outside the waveguide, sample with smaller air fraction shows more intense and well-defined structures.

Y. Benachour $e t . a l^{76}$ reported non-destructive characterization of planar two-dimensional (2D) photonic crystals $(\mathrm{PhCs})$ made in silicon on insulator (SOI) wafers using ellipsometric or Fourier transformed infrared (FTIR) spectroscope. At large wavelengths, devices behave as homogeneous isotropic materials defined by an effective filling factor. Even though they proved that diffractive optics methods, usually used in the short wavelength range to draw dispersion curves above the light cone, can operate in the long wavelength range to characterize actual PhCs.

\subsubsection{Characterization Set up}

Instead of using an optical setup with halogen lamp, polarizers, spectrometer and CCD camera to measure the variable specular reflectance of the photonic crystal above the light cone; FTIR( Fourier Transform Infrared Spectroscope) and Variable Angular Reflectance Spectroscopic (VASE) Ellipsometer were chosen. A brief study of the devices is discussed in the subsequent sections.

\section{(a)FTIR:-}

The term Fourier Transform Infrared Spectroscopy (FTIR) refers to a manner in which the data is collected and converted from an interference pattern of an optical spectrum. For the characterization of PhCs using the surface 
coupling technique, an additional accessory VEEMAX or VMAX (Variable grazing angle motorized stage), which is one of the specular reflectance accessory, is used in conjunction with normal FTIR operation. The optical design

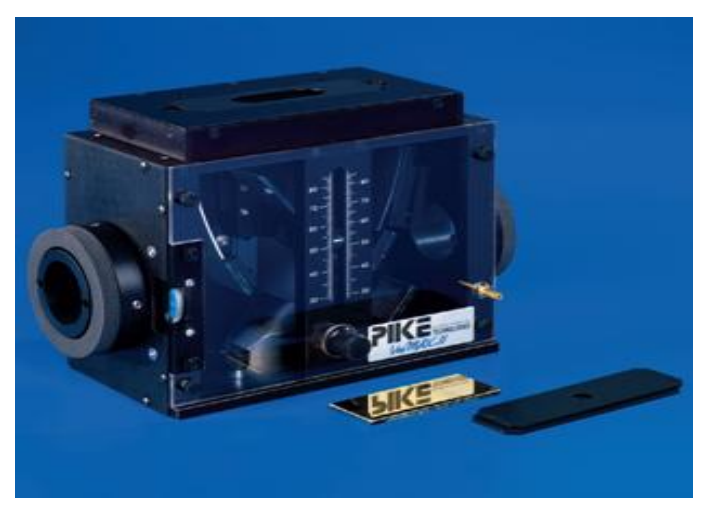

Figure 5.13 VEEMAX accessory ${ }^{77}$

enables the accessory to be in alignment for all angles of incidence. The angle may be varied continuously from $30^{\circ}$ to $85^{0}$ degrees by the rotation of a single control. The sample is placed face down on the sampling surface. Masks with $2 ", 5 / 8$ " and 3/8" apertures can be used to sample different geometries.

The setup can be used for collecting reflectance data in the mid-IR range. For operation at these wavelengths, the $\mathrm{PhC}$ lattice features are micron-scale. A major problem that occurred while using VEEMAX for varied angular specular reflectance measurements on a photonic crystal was the size of the sample. Sample sizes were very small 2-3mm, while the laser beam diameter which was much larger than the $\mathrm{PhC}$ pattern. As the angle is varied, the beam shifted its position of incidence on the sample (i.e beam was not incident on the same spot every time angle was varied,) which introduced significant uncertainty in the data collected, making it unreliable.

\section{(b)Ellipsometer}

An ellipsometer ${ }^{78}$ is capable of performing variable angular specular reflectance measurements, also known as VASE (Variable Angle Spectroscopic Ellipsometry). Ellipsometry is a very important technique to characterize the optical constants such a refractive index $n$ and extinction constant $k$ since the changes in the polarization state of the reflection beam from the sample are very sensitive to these parameters. In addition, ellipsometry is also a very useful technique to evaluate the properties of thin film multi-layers, where the polarization modification caused by the interference of the multiple reflected beams provides very accurate information about the thickness of the layers. 


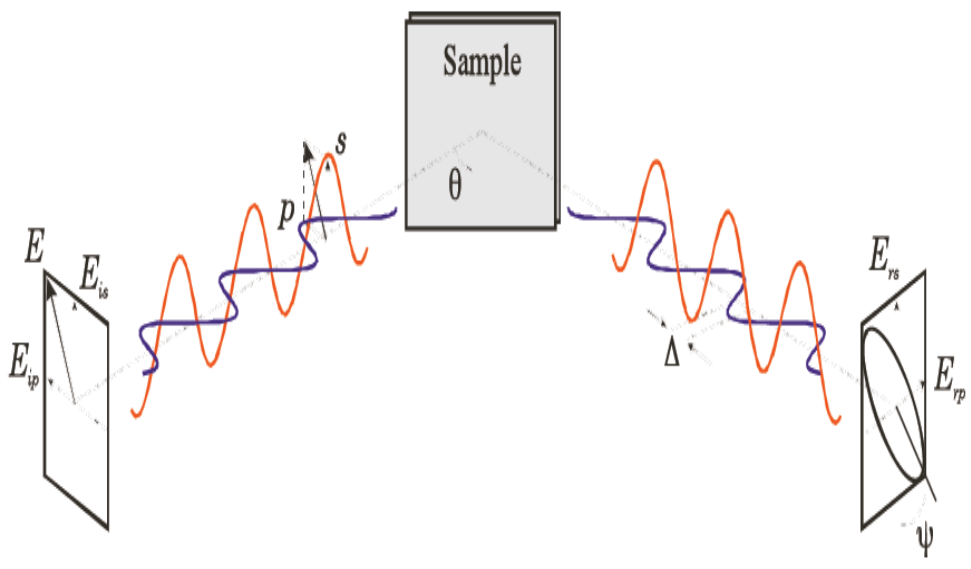

Figure 5.14 Principle of Ellipsometry ${ }^{15}$

The input arm or illuminating arm contains a quartz tungsten halogen (QTH) lamp, beam collimation optics and a polarizer with fixed orientation at $45^{\circ}$ with respect to the $s$ and $p$ components. Light reflected from the sample is then analyzed at the detection arm or output arm. The detection arm includes a rotating wave plate and analyzer, in order to achieve a full determination of the reflected beam polarization ellipse. In addition the detection arm includes a spectroscopic system that permits taking measurements at different wavelengths in the range of 290 to $996 \mathrm{~nm}$.

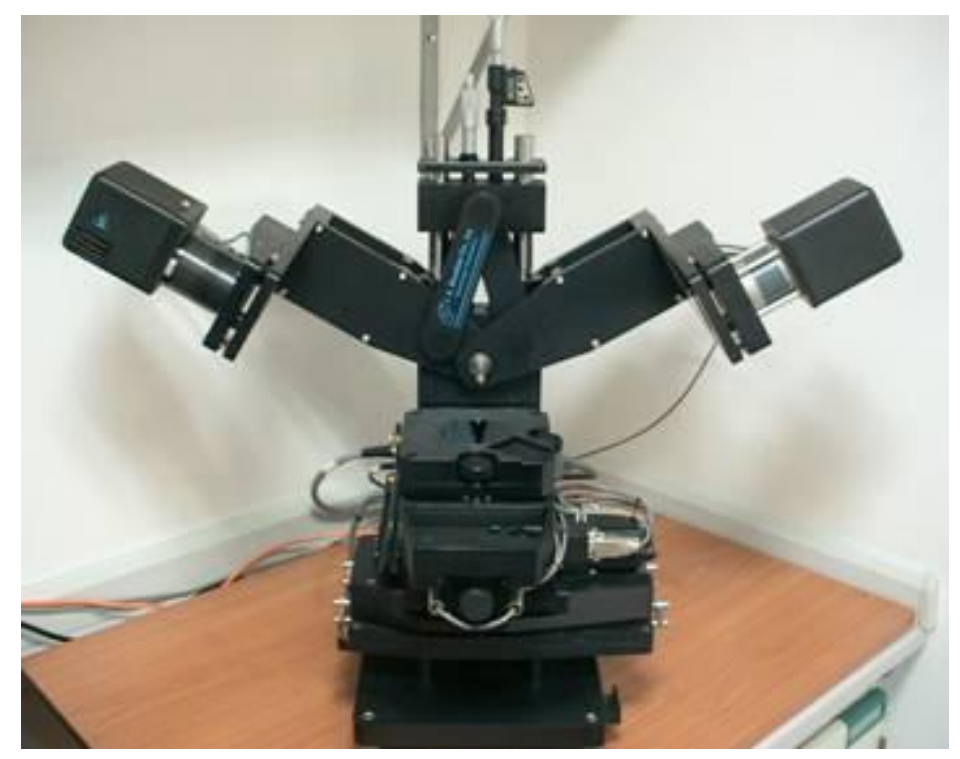

Figure 5.15 VASE setup ${ }^{79}$ 
In order to acquire variable angle specular reflectance data from the nano-scale $\mathrm{PhC}$ patterns with a surface area $500 \mu \mathrm{mX} 500 \mu \mathrm{m}$ of micrometers using the ellipsometer, lenses have to be inserted into the input and output arm. This lens setup helps in collimating the beam to a small beam areas of $\sim 300-600 \mu \mathrm{m}^{2}$. Though the lenses impose certain limitations such as the reduction in the power and the reduced angular range of incidence, variable angular reflectance measurements can be taken easily at different angles after calibrating the device with the lenses inserted. The variable angular specular reflectance data taken from a 1-D PhC patterned with a lattice constant of $1 \mu \mathrm{m}$ and air fractions of 0.3 on a SOI sample are shown in Figure 5.17. Patterns on SOI sample is a case of asymmetric PhC slab in which due to the asymmetric conditions, the bands are no more polarized as even or odd and bandgap vanishes. But, some of the experimental results in the literature showed that bandgap and the polarization exists even for asymmetric PhC slab such as SOI with air and silicon-dioxide as cladding layers when the refractive index contrast of the claddings is minimal ${ }^{80}$. Henceforth, two different samples were used for characterization; one with $\mathrm{PhC}$ patterns on SOI and one with suspended structures in Si. Initially, experimental data was taken on unpatterned SOI samples as a reference as shown in Figure 5.16. The fringes observed in the unpatterned case are different when compared to the patterned SOI.

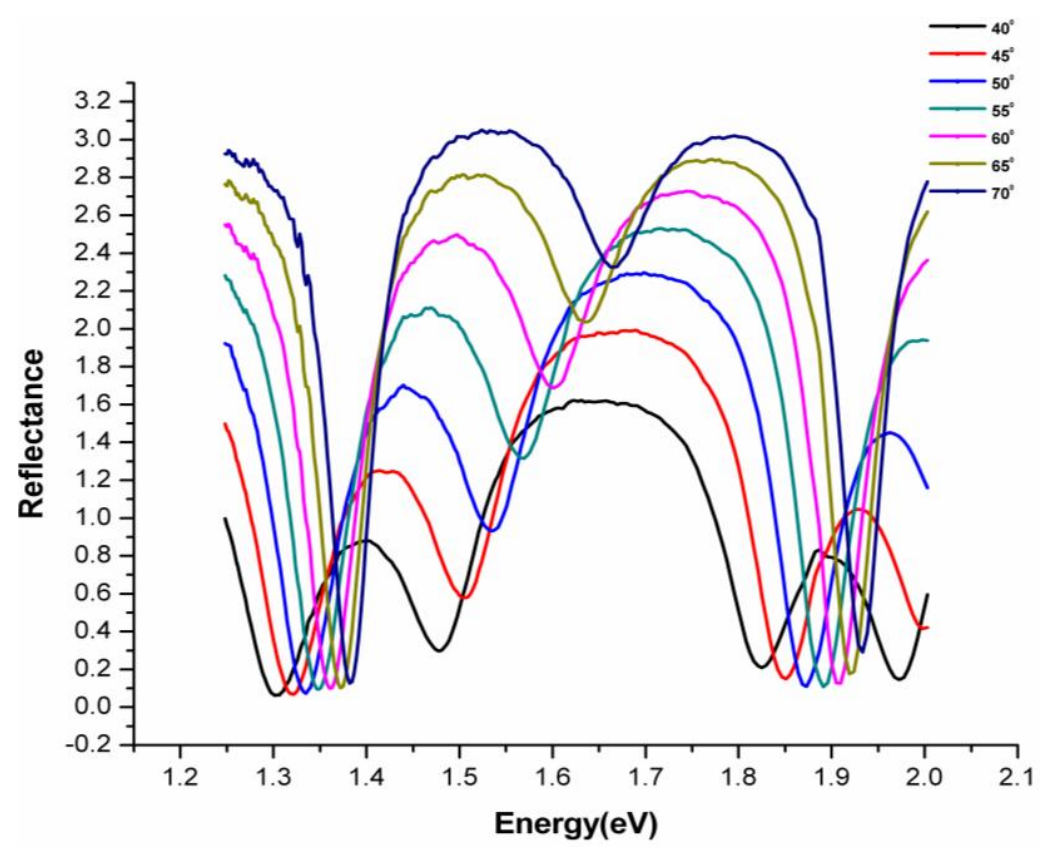

Figure 5. 16 s-pol reflectivity from unpatterned SOI substrate

The interference fringes in the diagram are caused due to the thickness of the layers on the substrate. In the patterned SOI, the resonant peaks are the guided modes evanescently coupled into the PhC pattern at the phase matching conditions. 


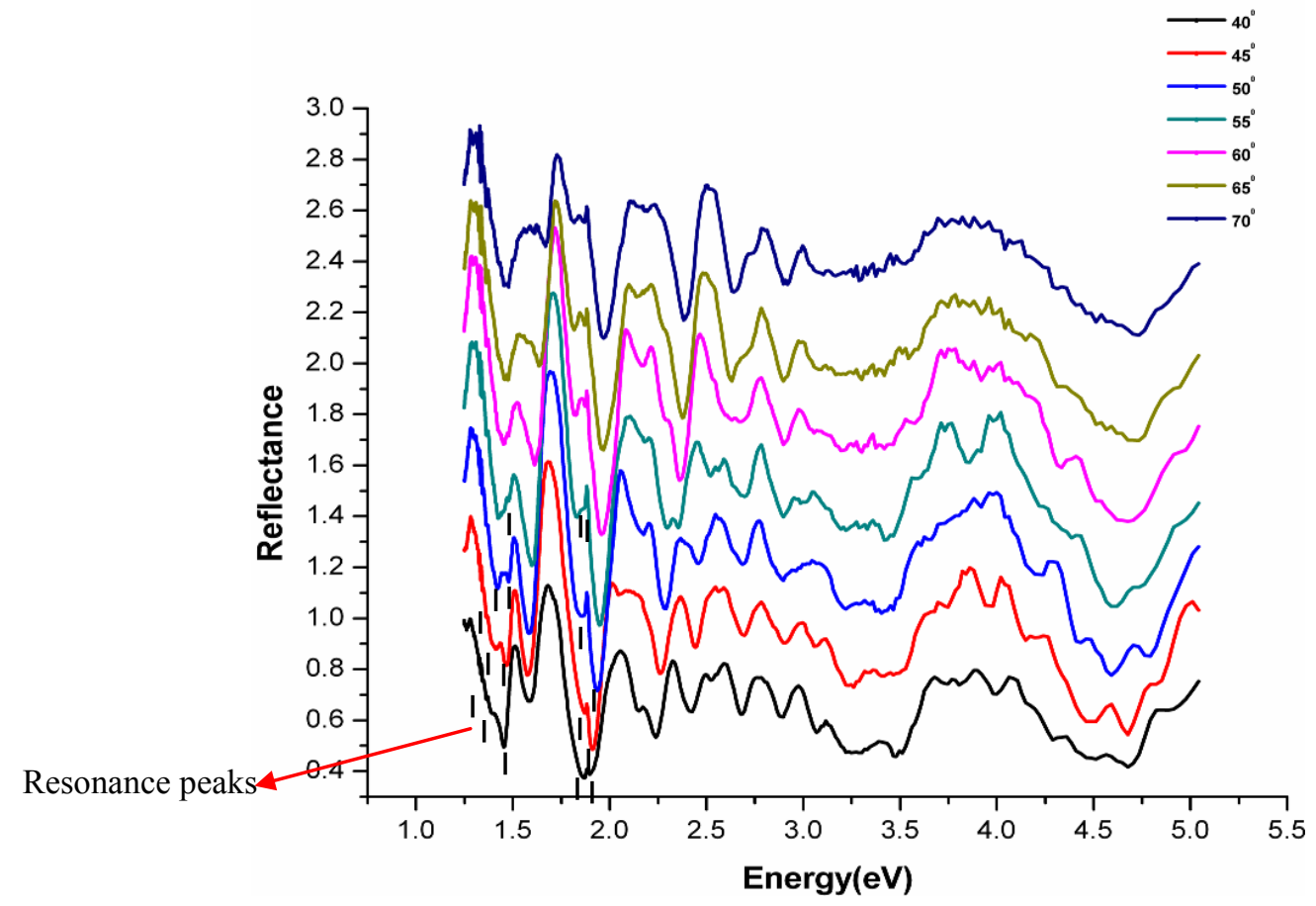

Figure 5.17 s-pol reflectivity spectra of 1-D PhC in SOI (700_300nm) wafers

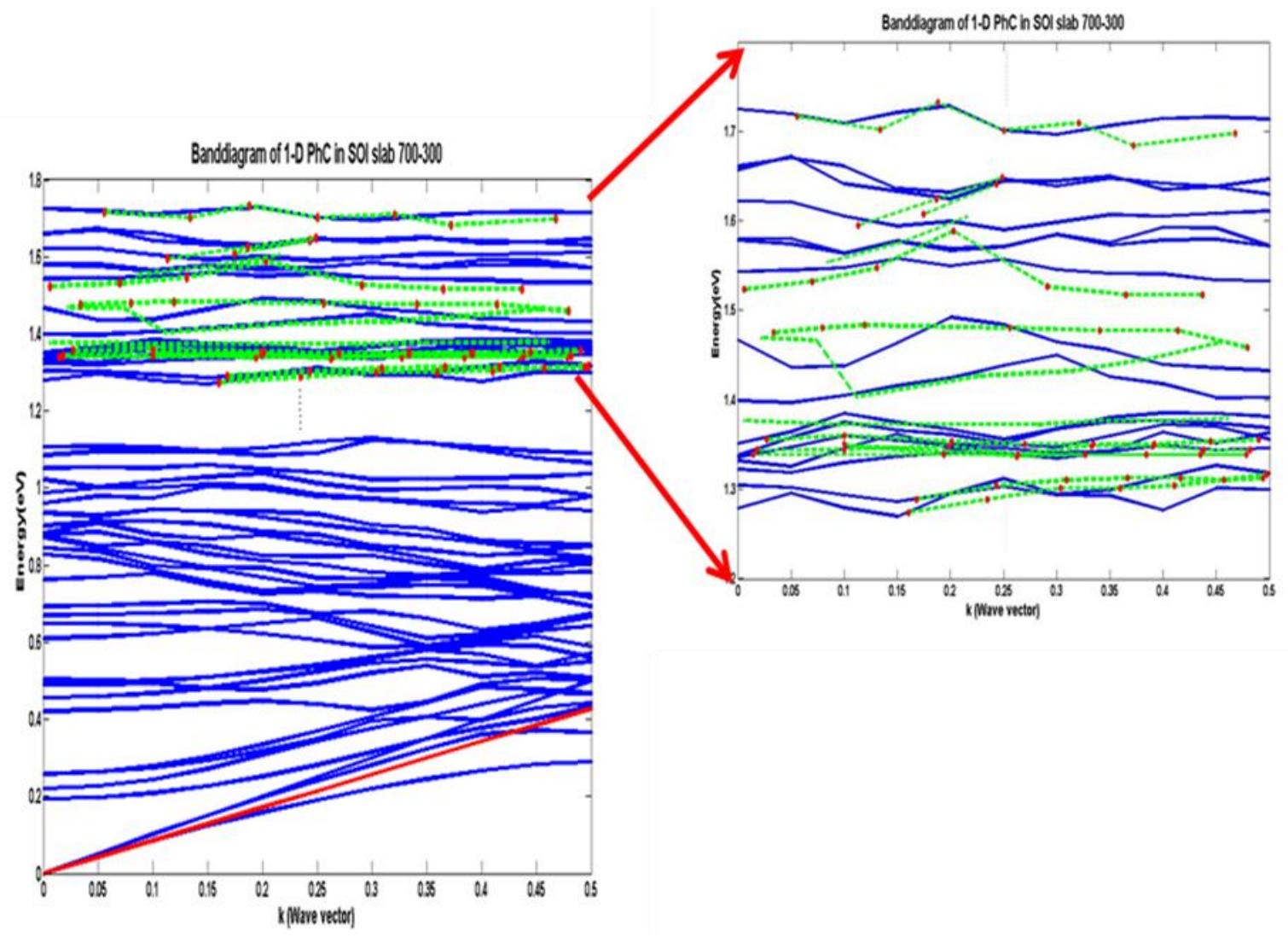

Figure 5. 18 Theoretical and experimental data from the surface coupling technique plotted 
The energy positions at the resonant peaks or inflection points in Figure 5.17 were chosen and their corresponding in-plane wave vector is calculated using Equation (5.1).

$$
k_{\|}=\frac{\omega}{c} \sin \theta
$$

The resonant peaks at different angles are mapped onto the band diagram of the 1-D PhC slab as shown in Figure 5.18. In Figure 5.18, theoretical band-diagram was calculated using MPB. The band diagram obtained from MPB will have $y$-axis as normalized frequencies. Since we are using an ellipsometer which gives data corresponding to energy of the photon, the frequency band diagram was converted to energy band diagram. Figure 5.17 shows the radiative modes extending to $1.8 \mathrm{eV}$, and the radiative modes are a continuum where no bandgap exists. Plotting the experimental ellipsometer data and theoretical band structure on the same graph indicated close correlation of experimental and theoretical results. The graph indicates that there is good agreement with theoretical and experimental data except that there are few variations which are mostly due to the following:

1. The 1-D PhC pattern fabricated on the samples does not extend infinitely. The pattern consists of $100 \mathrm{X} 100 \mu \mathrm{m}^{2}$ patterns stitched together to form a $600 \mathrm{X} 600 \mu \mathrm{m}^{2}$ area to meet the requirement of lens of Ellipsometer whose beam width is around the $\sim 500-600 \mu^{2}$. The difference in the modes may be due to the stitching issues in the fabrication.

2. Also the data was collected from $40^{\circ}$ to $70^{\circ}$ at regular intervals of $5^{\circ}$. If the data is taken at a wider range of angles at higher resolution, more accurate results would have been obtained.

To avoid the limitations on the bandgap caused by the asymmetric nature of photonic crystal slab in SOI, surface coupling is done on a sample with $\mathrm{PhC}$ patterns in suspended $\mathrm{Si}$ (with air cladding). The corresponding experimental data and the mapping between theoretical and experimental data are shown in Figure 5.19 and 5.20 respectively.

Figure 5.19 shows the data taken between $40^{\circ}$ to $70^{\circ}$ at regular intervals of $5^{\circ}$ with a s-polarized light which is similar to TE polarized light. Figure 5.20 shows the mapping between theoretical and experimental data of a 1-P PhC suspended in air much above the light cone. 


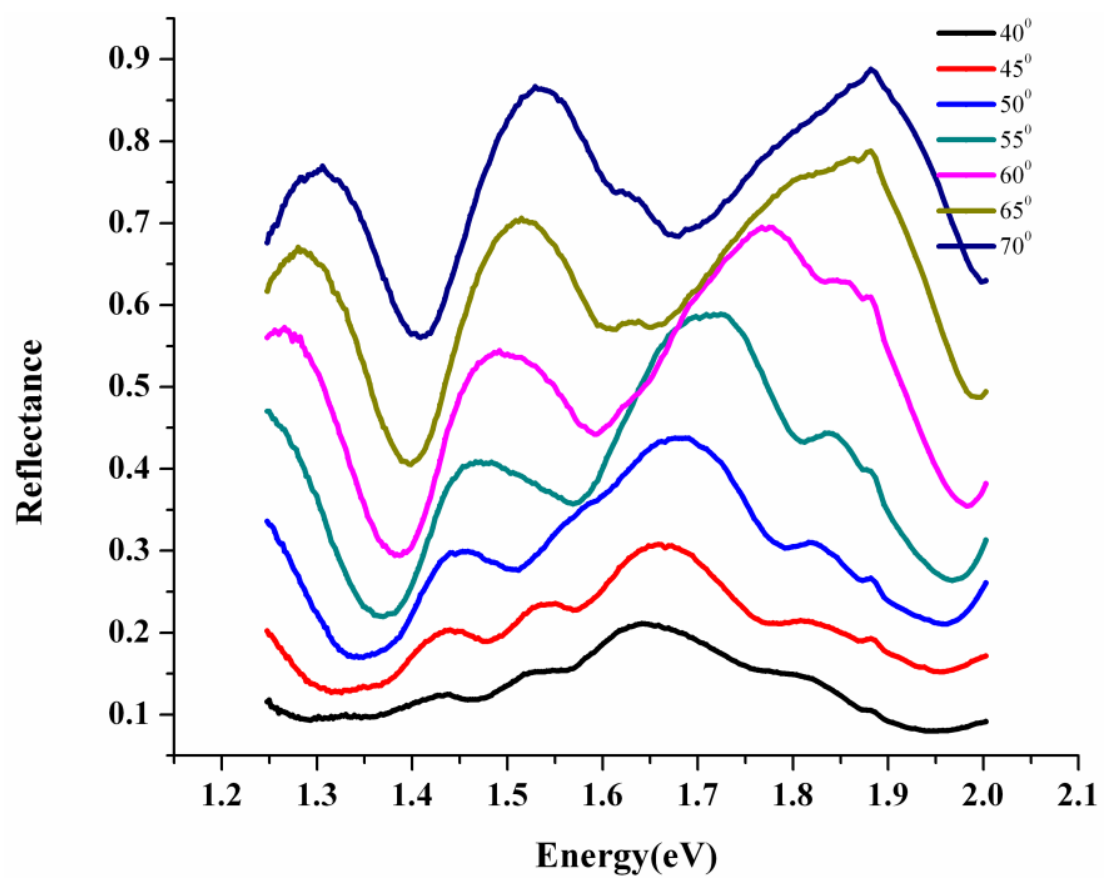

Figure 5. 19 spol reflectivity spectra of suspended $\mathrm{PhC}$ in $\mathrm{Si}$ (700_300nm lattice)

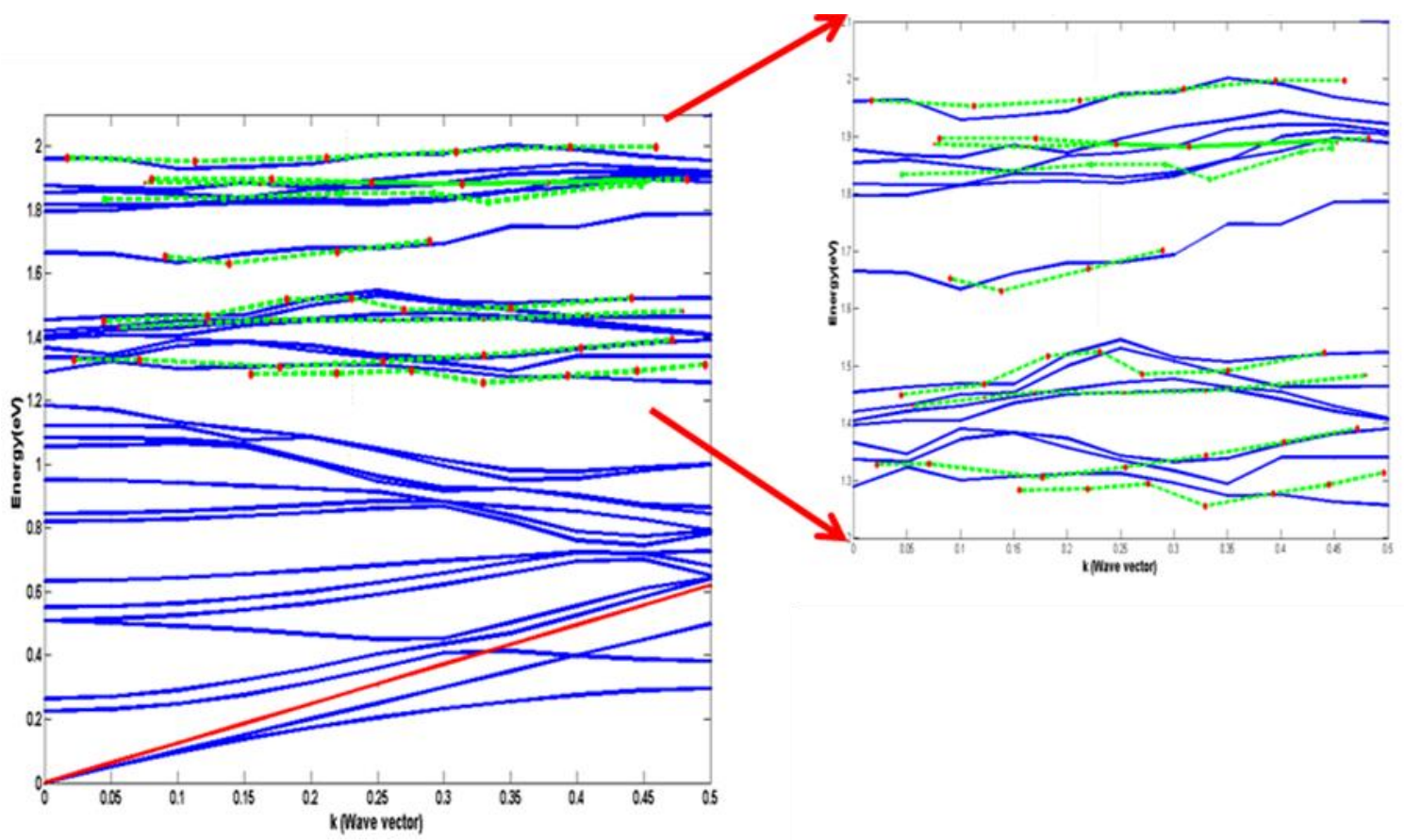

Figure 5. 20 Zeven band diagram and experimental data from 1-D PhC suspended in air with lattice constant of $1 \mu \mathrm{m}$ 
These results indicate that band diagram far above the light cone can only be drawn using this technique. In order to verify the band diagram near the light cone; a lamp which operates near the bandgap is needed. As an alternative, the lattice parameter could be reduced, allowing modes nearer to the light cone to be plotted.
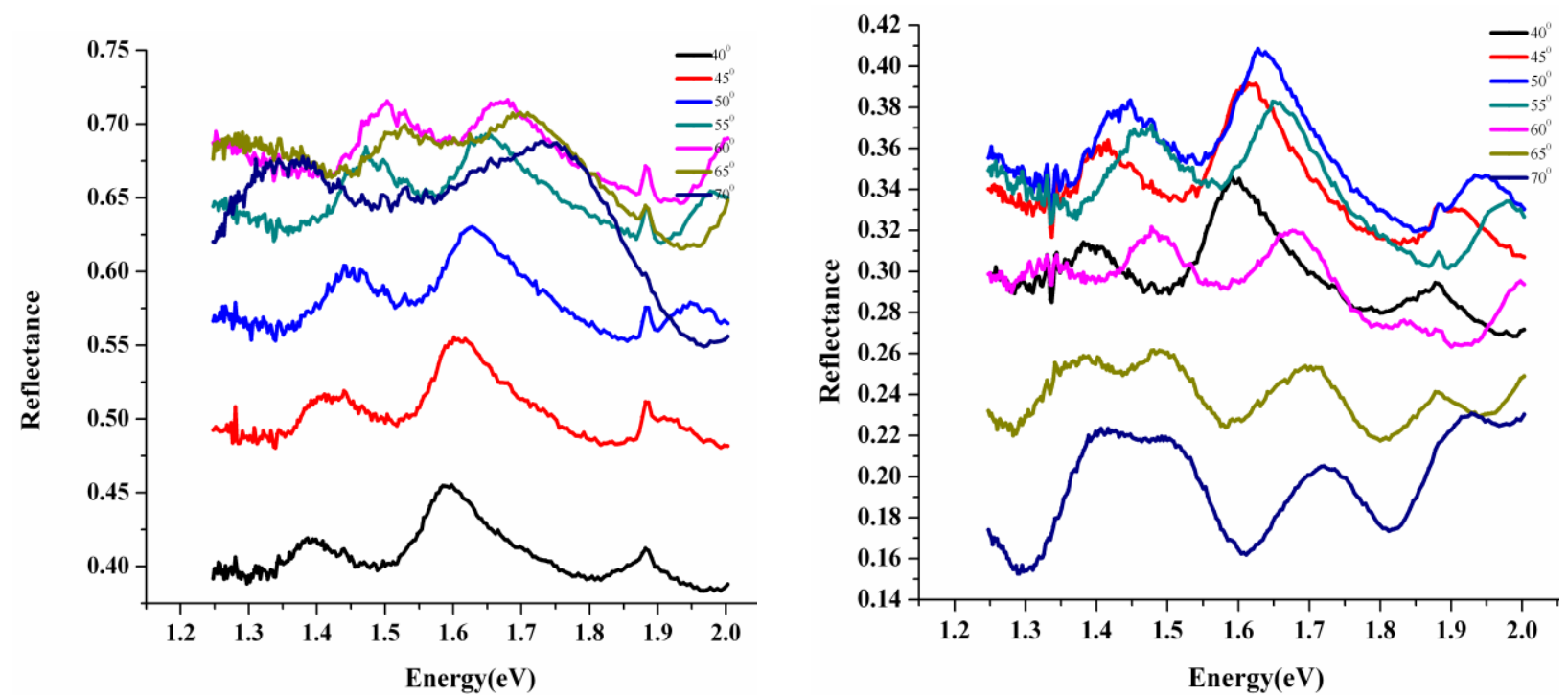

Figure 5. 21 spol and ppol reflectivity spectra of 1-D PhC suspended in air in400_200nm lattice respectively
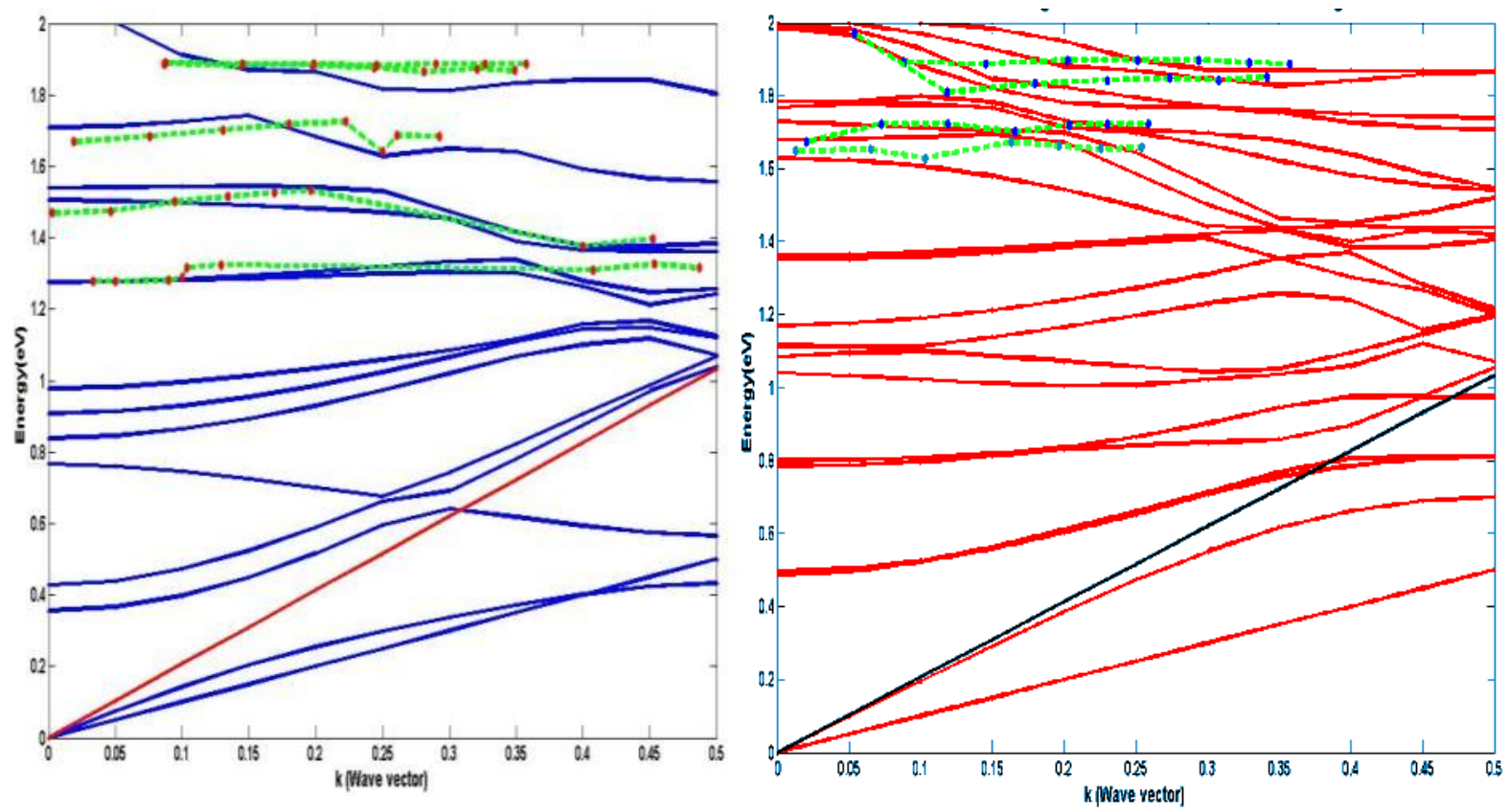

Figure 5.22 zeven and zodd Band Diagram of 1-D PhCs in Si suspended in air with lattice constant 600nm

To verify this, another sample with a $600 \mathrm{~nm}$ lattice constant and $400 \mathrm{~nm}$ stripe widths were fabricated and measured using the ellipsometer as shown in Figure 5.21. The pattern is suspended in Si to avoid asymmetrical conditions. Figure 5.22 indicates zeven and zodd modes mapping onto a theoretical band diagram obtained using MPB. The 
modes closer to the light cone can thus be measured by reducing the lattice parameter size. In future work, further reduction of lattice size would allow radiative modes directly outside the light cone to be measured using the existing ellipsometer light source.

\subsection{Optical Characterization of Waveguides}

The dimensions derived from modeling tools were used for fabricating a 2-D $\mathrm{PhC}$ which operates in the visible spectral range. To implement a GaN based 2-D PhC as a in biosensing architectures ${ }^{6,7}$, light from the laser must be coupled into the GaN slab waveguide. Since this waveguideing is a critical function of our sensor design, the device functionality may fail if the optical properties of $\mathrm{GaN}$ are not optimal or if the $\mathrm{GaN}$ slab doesn't act as a waveguide. Of these, the latter problem can be solved when the slab is grown with optimum values of thickness which allow coupling of light into waveguide, whereas the former problem can be solved if a high quality film is grown in the lab. Losses of $\mathrm{GaN}$ films should be determined in order to optimize film growth for optical operations as a waveguide. The next section covers loss mechanisms in detail.

\subsubsection{Prism Coupling Characterization:}

A prism coupler is a device used to couple a laser beam efficiently into thin-film dielectric wave guides to determine the refractive index and thickness of the guiding film, provided that the film supports two or more modes. Prism Coupler operation is based on frustrated total internal reflection ${ }^{17}$. When light is incident from a higher index material to a lower index material, there is total internal reflection if the incident angle exceeds critical angle. Though there is no power in the second medium there is an evanescent wave which decays rapidly. If there is a third medium which is optically denser than the second medium, and if the thickness of second medium is not large enough to decay the evanescent wave (total internal reflection gets frustrated) then the power from first medium is transferred to the third medium.

A Metricon2010/M prism coupler setup is shown in Fig 5.22. It shows a laser beam (light source) that hits the high indexed prism during which some of the light passes into the film through the air gap. The evanescent fields that pass in to the film are capable of transferring power between the incident beam and a waveguide mode. A pneumatic plunger holds the sample in close vicinity to the prism and a stepper motor is used to rotate the prism/plunger assembly while a stationary laser is incident on the rotating prism face. The rotating assembly allows for coupling into the waveguide sample's discrete modes. 


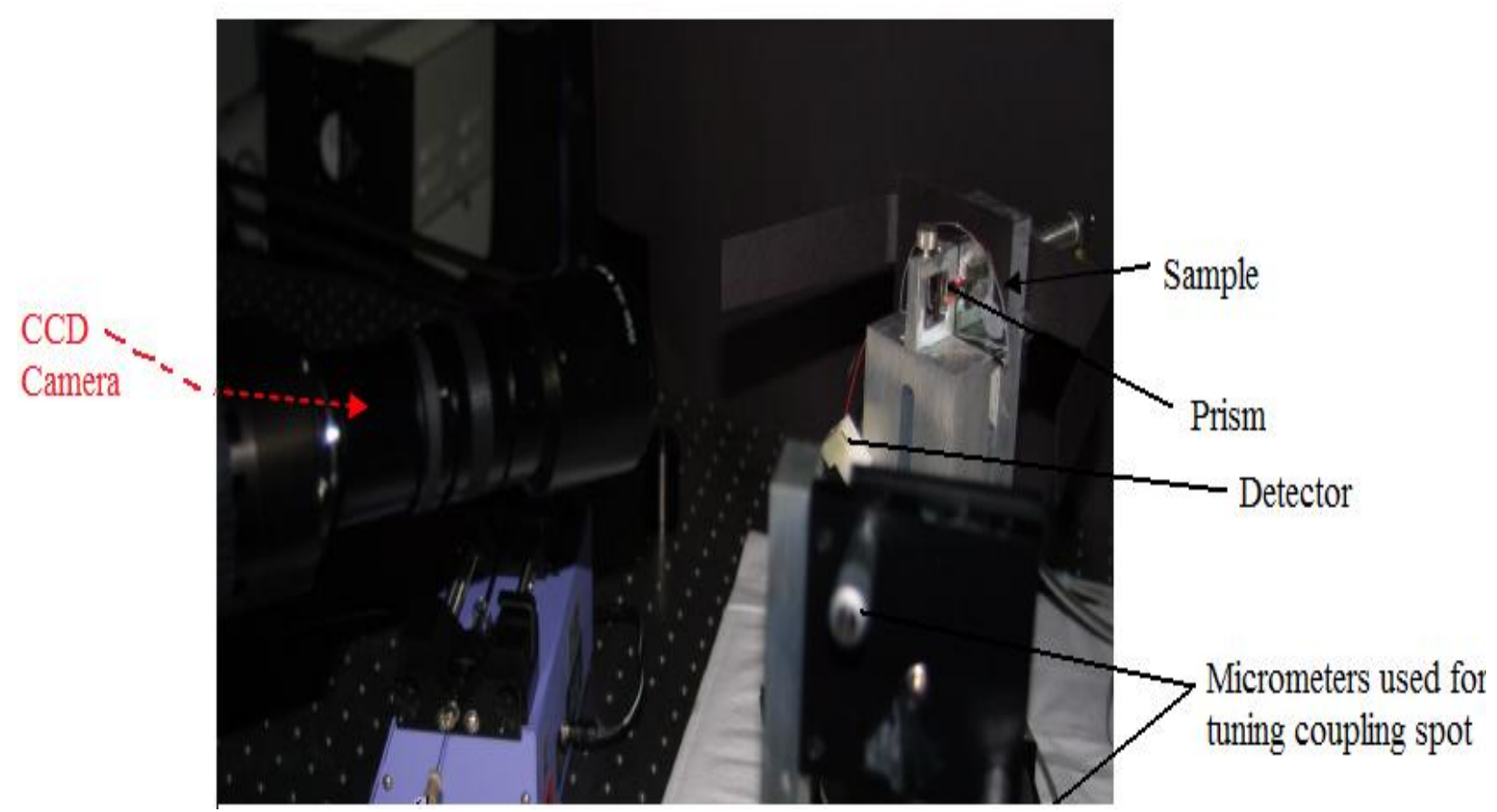

Figure 5.22 Metricon 2010/M Prism coupler setup ${ }^{14}$

Every mode in a waveguide, depending upon the medium, has a distinct angle at which the light propagates. If the light is incident on the prism at an angle greater than critical angle, total internal reflection takes place and the light gets reflected as shown in Fig 5.23.

\section{Effective Refractive Index:}

Rays traveling in each mode angle have a corresponding modal index which is also known as effective index. This is also defined as the free-space velocity divided by the guided wave velocity

$$
n_{e f f}=\frac{c}{v_{g}}=n_{2} \sin \theta_{2},
$$

where $\theta$ is the angle of incidence on the face of the prism. Ray angles for propagating waves vary from the critical angle to $90^{\circ}$ and thus, the $n_{\text {eff }}$ value lies somewhere between the core and cladding refractive indices. Coupling losses of any sample can be calculated using this setup. 


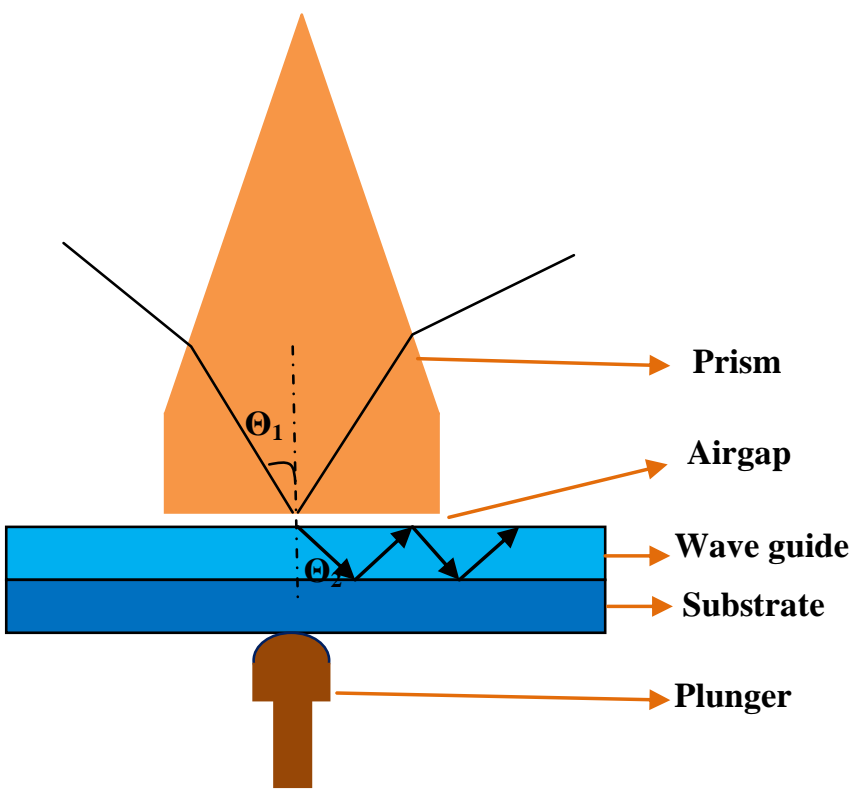

Figure 5.23 Cross -sectional view of the prism coupler layout

The GaN slab was grown on a thin AlN layer ( $\sim 1 \mathrm{~nm})$ on top of a sapphire substrate to a thickness of approximately $2 \mu \mathrm{m}$. As the prism couples light into the sample, it produces a streak which indicates a propagating mode. Figure 5.24 shown below is a streak in the sapphire substrate.

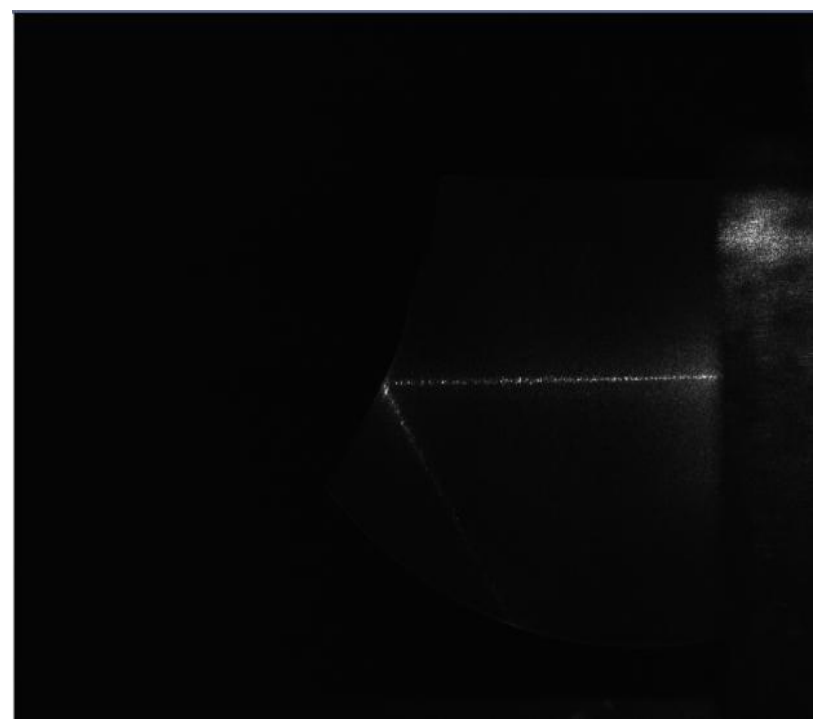

Figure 5.24 Picture of streak in sapphire from CCD camera 
The second mode obtained was observed using the CCD camera as the beam incidence angle was varied. The image taken from the CCD camera was used as an input into a Matlab script ${ }^{16}$ that converts the image into RGB, and, when a portion of the streak is selected, generates plots that are used to characterize waveguide loss (intensity vs. length, $\log$ of intensity vs. length and curve fitting tool). The slope of the curve-fitted line is calculated which gives value of

$$
\text { Coupling losses }=-4.3 \alpha
$$
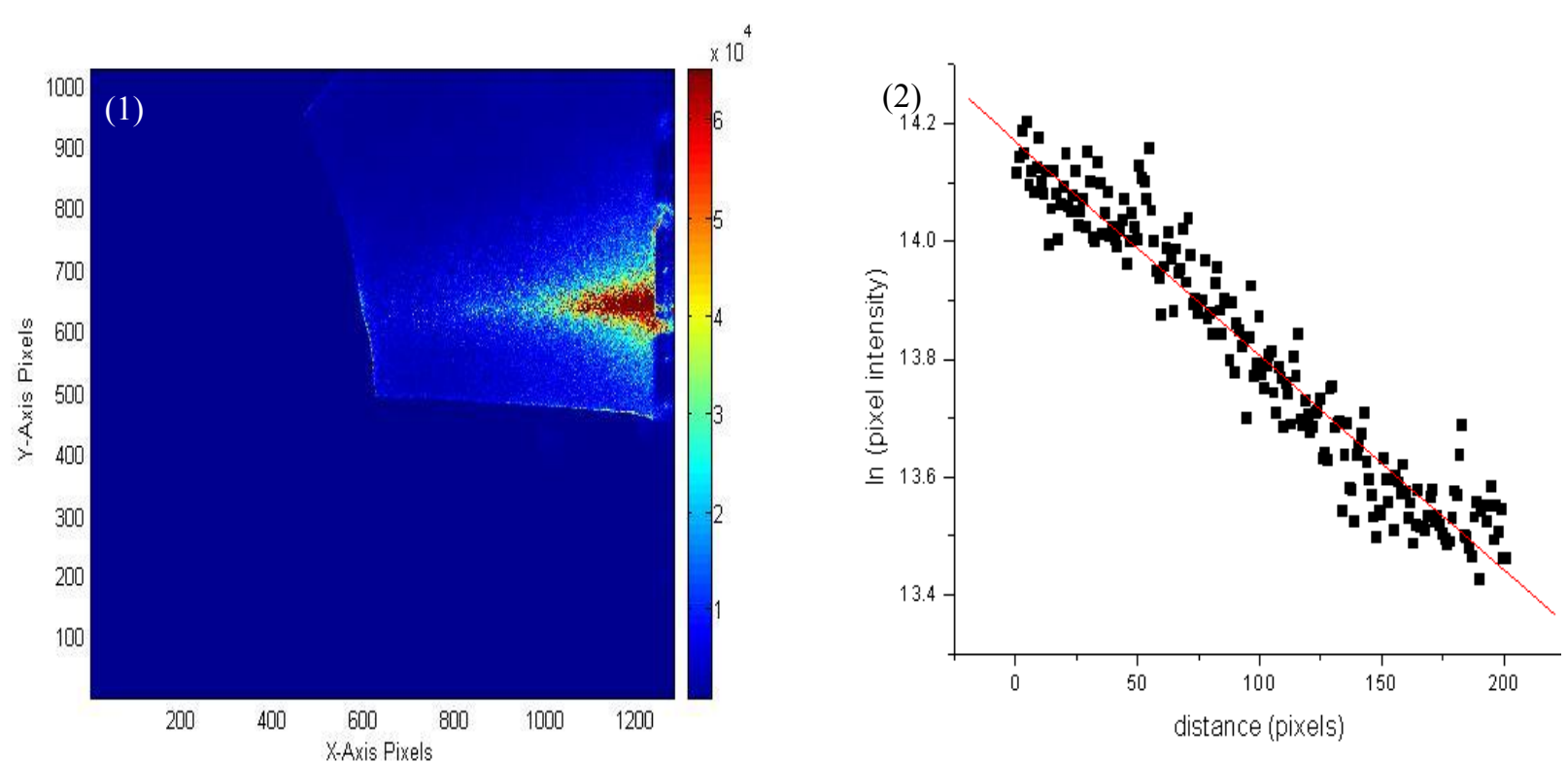

Figure 5.24(a) The guiding streak through the GaN slab. (b) The plotted intensity decay across the propagating distance

The coupling losses for a sapphire substrate were found to be $2.02 \mathrm{db} / \mathrm{cm}$ and coupling losses in $\mathrm{GaN}$ were found to be $4.01 \mathrm{db} / \mathrm{cm}$. The losses indicated that the GaN film growth conditions need more optimization.

\subsection{SUMMARY}

Reflectance data taken from Ellipsometer using surface coupling technique which was in good agreement with the theoretical data except a few variations due to pattern area, interference due to background and low range of angles. 1-D PhCs on a SOI substrate as well as suspended patterns were successfully characterized for the modes above light cone with an ellipsometer. To plot the band diagram near the light cone, lattice parameters size was reduced and the data extracted from the graph was near the light cone. The bandgap of the PhC, which lies below light cone, can also be characterized using ellipsometer if the device is equipped with a light source which operates in the wavelength range of $1 \mu \mathrm{m}-3 \mu \mathrm{m}$. Coupling losses of $\mathrm{GaN}$ on sapphire were successfully determined using Prism coupler setup which indicated that optimization in the growth process is required to achieve better results. 


\section{Conclusions and Future Work}

\section{Conclusions}

In this research effort, bandgap tuning capabilities of PhCs in III-V nitride thin films were explored. Initially, 2-D PhCs in AlN thin films were considered for tuning. Finite element modeling results of nanometer patterns of circular air holes on AlN indicated picometer scale deformation in the lattice parameters. This insignificant lattice parameter change would be hard to measure, as the errors in fabrication using E-beam Lithography are considered to be greater than the geometrical variation due to piezoelectric effects. Furthermore, the change in lattice parameter due to deformation is not enough to result in an appreciable change in theoptical bandgap. However, a clamped-clamped beam (without holes) repeated periodically (essentially a 1-D PhC) suspended in air shows greater promise for increased deformation. FE Analysis of 1-D PhCs showed significant out-of-plane deformation in the overall slab surface which is considered to be $\sim 10-20$ times larger than the deformation in the lattice. This effect can potentially be used for tuning of bandgap by effectively changing the angle of incidence for a 1-D PhC. Extensive modeling on the impact of off-axis incident light on the effective bandgap substantiated this claim. FEA studies indicated that the deformation of 1D PhCs in AlN films with low applied voltages lead to deformations on the order of picometers, which were not large enough to affect the optical bandgap. Application of higher voltages and frequencies which create resonance phenomena in the structure can create a maximum slab deflection of $\sim 120 \mathrm{~nm}$. Theoretical optical modeling results indicated that $7 \mathrm{~nm}$ deflection is sufficient for creating a $2-4 \%$ change in the bandgap, which can be acquired by applying a voltage of $30 \mathrm{~V}$.

Metrology equipment (both mechanical/piezoelectric and optical) limitations two differently scaled 1-D PhCs were fabricated for characterization. Due to restrictions on the characterizing devices, nanometer scale 1-D PhCs were fabricated in $\mathrm{Si}$ as well as in AlN for optical characterization (at 1.55 microns) and micron scale 1-D PhCs were fabricated in AlN for mechanical characterization. Even though nanometer scale 1-D PhCs were successfully fabricated in Si substrate and AIN thin films which can be used for optical characterization, fabrication of suspended micron-scale 1-D PhCs in AlN resulted in buckling effect which might be accounted to the stress in the sputtered AlN thin films. This indicates that the process for sputtering AIN needs further optimization in order to create suspended AlN 1-D PhCs on SOI substrates.

A surface coupling technique was used to characterize photonic crystals for plotting the radiative modes. Ellipsomtery was used to acquire reflectance data using variable angular incidence. The experimental data was in 
good agreement with the theoretical data except a few variations due to pattern area, and the angular resolution of the measurments. 1-D PhCs on a SOI substrate, as well as suspended patterns, were successfully characterized for the modes above light cone. However, the modes acquired for such structures were far away from the light cone due to the available source wavelength. In order to plot the radiative modes near the light cone, lattice parameters size was reduced. The manual mapping of the experimental data to the theoretical data indicated the modes for this structure existed much closer to the light cone. The bandgap of the $\mathrm{PhC}$, which lies below light cone, can be characterized using an ellipsometer with a light source which operates in the wavelength range of bandgap.

\section{Future Work}

One of our future work that relates to this research includes process optimization for sputtering AlN to create suspended 1-D PhCs for mechanical characterization. Also, a metrology setup is required to simultaneously characterize PhCs optically and mechanically incidentally to explore tuning capabilities. Even though the modeling results indicated efficient bandgap tuning with out-of plane deformation of $\mathrm{PhC}$ structure, such bandgap tuning mechanism may limit PhC applications.

Another avenue for future work involves considering other mechanisms for bandgap tuning in piezoelectric PhCs. Instead of using physical deformation of the device to tune the bandgap, a novel idea involving the associated change in refractive indices due to the application of voltage to a piezoelectric material can be further explored for effective bandgap tuning. The master equation which gives eigen-solutions for a $\mathrm{PhC}$ structure was derived assuming free charge carriers and current densities to be zero in an isotropic medium. But, in an anisotropic piezoelectric material, application of electric field induces change in the polarization which effects dielectric permittivity of the material. The dielectric constant of a material is proportional to $\boldsymbol{n}^{2}\left((\text { refractive index })^{2}\right)$. This change in refractive index can be used to potentially explore the bandgap tuning capabilities of PhCs in nitride thin films. 


\section{APPENDIX A: PRISM COUPLER OPERATION}

1. Mount the sample onto the Prism coupler and reference it if concerned with the beta values of the film.

2. Once the effective indexes are calculated remove the detector and place the CCD camera focusing on the regions you like to measure.

3. Place a graph paper on the back side of the sample and take measurement with light switched on so that it can be used as a reference for pixel to distance conversion.

4. Without changing the focus of the camera take the images for different exposure times.

5. Always the prism's index has to be greater than the film index.

6. Prism used in the experiment has an index of 2.8.

7. Due to this high index of prism, reference gain raised above the scale. This gain was reduced using beam splitters, and filters. A filter which when used could bring the gain exactly to an optimum value was not found which results in very low gain. Because of these reasons $n_{e f f}$ and thicknesses were not calculated from prism coupler measurement instead they were calculated from ellipsometer .This setup was only used to calculate losses. 


\section{APPENDIX B: Diffraction Grating Theory}

When light is incident on a grating surface, each grating or groove becomes a source of transmitted/reflected light. At a unique set of angles, light scattered from the facet is in phase due to constructive interference. This occurs when the geometrical path difference between successive grooves equals the wavelength of light and the light will be in phase. At all other angles, the light is out of phase due to destructive interference. The grating equation which describes these diffraction phenomena is given by ${ }^{81}$

$$
\operatorname{Sin} \theta_{m}=\operatorname{Sin} \theta_{i}+m \frac{\lambda}{d} ; \quad m=0, \pm 1, \pm 2 \ldots . .
$$

Where $\theta_{i}$ (and $\theta_{m}$ ) are the angles between the incident (and the diffracted) wave directions and the normal to the grating surface which is assumed perpendicular to the plane of grooves, $\lambda$ is the wavelength and $d$ is the grating period (Fig.a). $m$ is an integer. When $m=0$, the grating acts as a mirror. If the plane of incidence is not perpendicular then Equation 5.1 transforms as

$$
\boldsymbol{k}_{m_{x}}=\boldsymbol{k}_{i_{x}}+\boldsymbol{m} \frac{2 \pi}{d} \quad \text { and } \quad \boldsymbol{k}_{m_{z}}=\boldsymbol{k}_{i_{z}}
$$

Equation 5.2
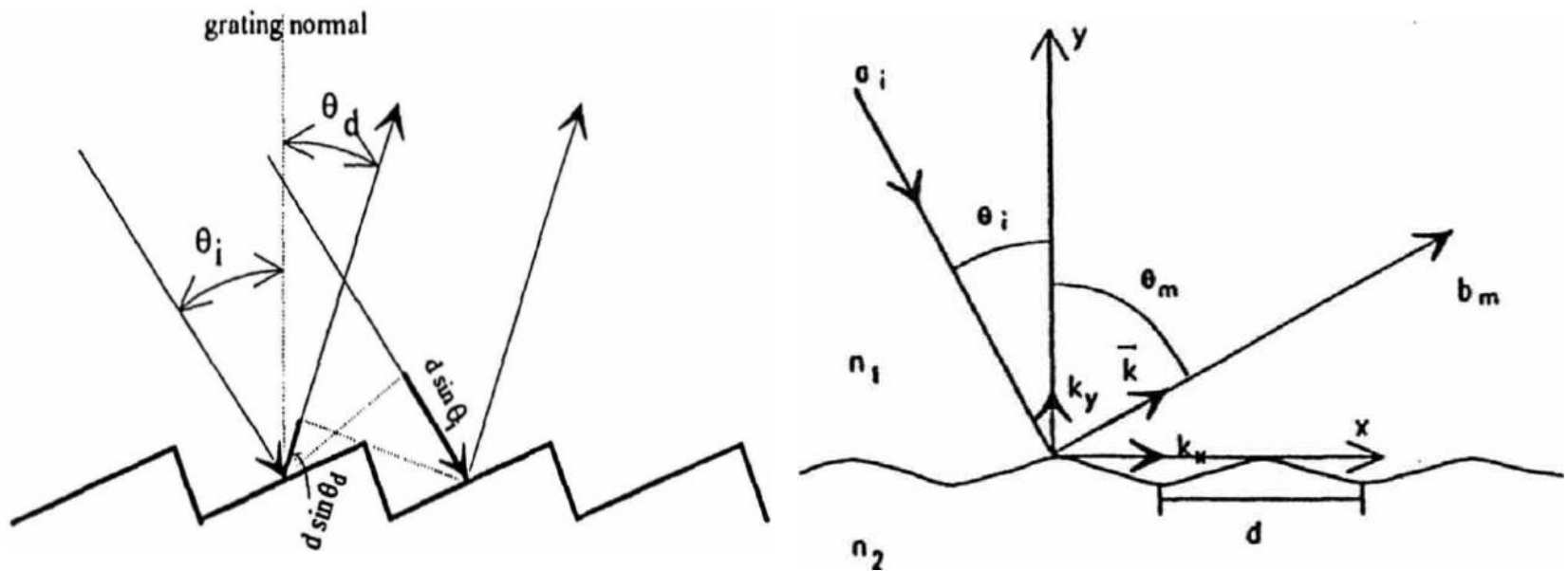

Figure( a) Diffraction grating ${ }^{82}$ Figure (b) Schematic of diffraction grating in co-ordinate axis ${ }^{82}$

where $k_{x}, k_{y}$, and $k_{z}$ are the wave vector components of the plane wave in $\mathrm{x}, \mathrm{y}$ and $\mathrm{z}$ directions. For a transmission grating, direction of propagation of the transmission orders can be determined by

$$
k_{x}=n \frac{2 \pi}{\lambda} \sin \theta
$$

It is also necessary to distinguish between the polarizations of the wave that is incident on the grating. If the incident wave is linearly polarized and the electric field vector is perpendicular to the plane of incidence, all the diffracted 
orders have the same polarization. It is called s, or P, or TE polarization. The other case, when the electric field lies in the plane of incidence, also preserves the polarization direction and is called $\mathrm{p}$, or $S$, or the TM case.

The grating equation can be satisfied for more than one value of $m$, but the solution exists only when

$$
\left|\sin \theta_{m}\right|<1
$$

Equation 5.2

The diffraction orders with number $m$ such that the condition is fulfilled are called propagating orders. The vertical wave vector component can be easily found from the wave equation which is real

$$
\boldsymbol{k}_{x}^{2}+\boldsymbol{k}_{y}^{2}=\left(\frac{2 \pi}{\lambda} n\right)^{2}
$$

For the orders with $m$ which satisfies the condition $\left|\sin \theta_{m}\right|>1$ the vertical wave vector component derived is imaginary. i.e., these orders decrease exponentially with the distance from the grating surface. These orders are called evanescent orders. They cannot be detected at a distance larger than few wavelengths from the surface of the grating, but can play an important role in surface-enhancement properties. The property of gratings to couple infinite number of electromagnetic waves plays an important role in integrated fiber optics and waveguides. The guiding property in a waveguide prevails only when the wave is in propagating order in the core and evanescent order in the cladding layers. The property of the grating to couple evanescent or radiative orders to propagating orders (in the cladding) is widely used to couple light into and out of the waveguide.

The mode is characterized by its propagation constant, phase velocity $k_{G}$ in the direction of propagation, which takes discrete values depending on the waveguide optical and geometrical properties such as the wavelength, and polarization. This constant should always be greater than the modulus of the wave vector in the cladding so that the radiation field in the cladding is evanescent. According to the equation of propagation and wave vectors of a evanescent wave, the grating can couple this evanescent wave into a propagating order under specific conditions called phase-matching conditions given by,

$$
k_{G}=\frac{2 \pi}{\lambda}\left(n_{1} \sin \theta_{m}+m \frac{\lambda}{d}\right)
$$

Diffraction gratings can be fabricated in many ways. Metallic and dielectric gratings are a few examples. Our main interest is dielectric gratings. Dielectric gratings are constructed of dielectric materials that are transparent to electromagnetic radiation that impinges on it. Scattering from dielectric diffraction gratings depends strongly on three main factors, namely the type and strength of periodic variation of the refractive index, the type of material the grating is made from, and the type of EM wave that is incident on the grating ${ }^{82}$. Of these factors periodic variation of the grating may be one-dimensional or it may be two-dimensional referred to a crossed grating. 
Taking into account the phase matching conditions and the periodic variability of gratings, the laws derived in this section can be used for drawing dispersive curves of either 1-D or 2-D PhCs above the light cone by using surface coupling techniques ${ }^{83}$. 


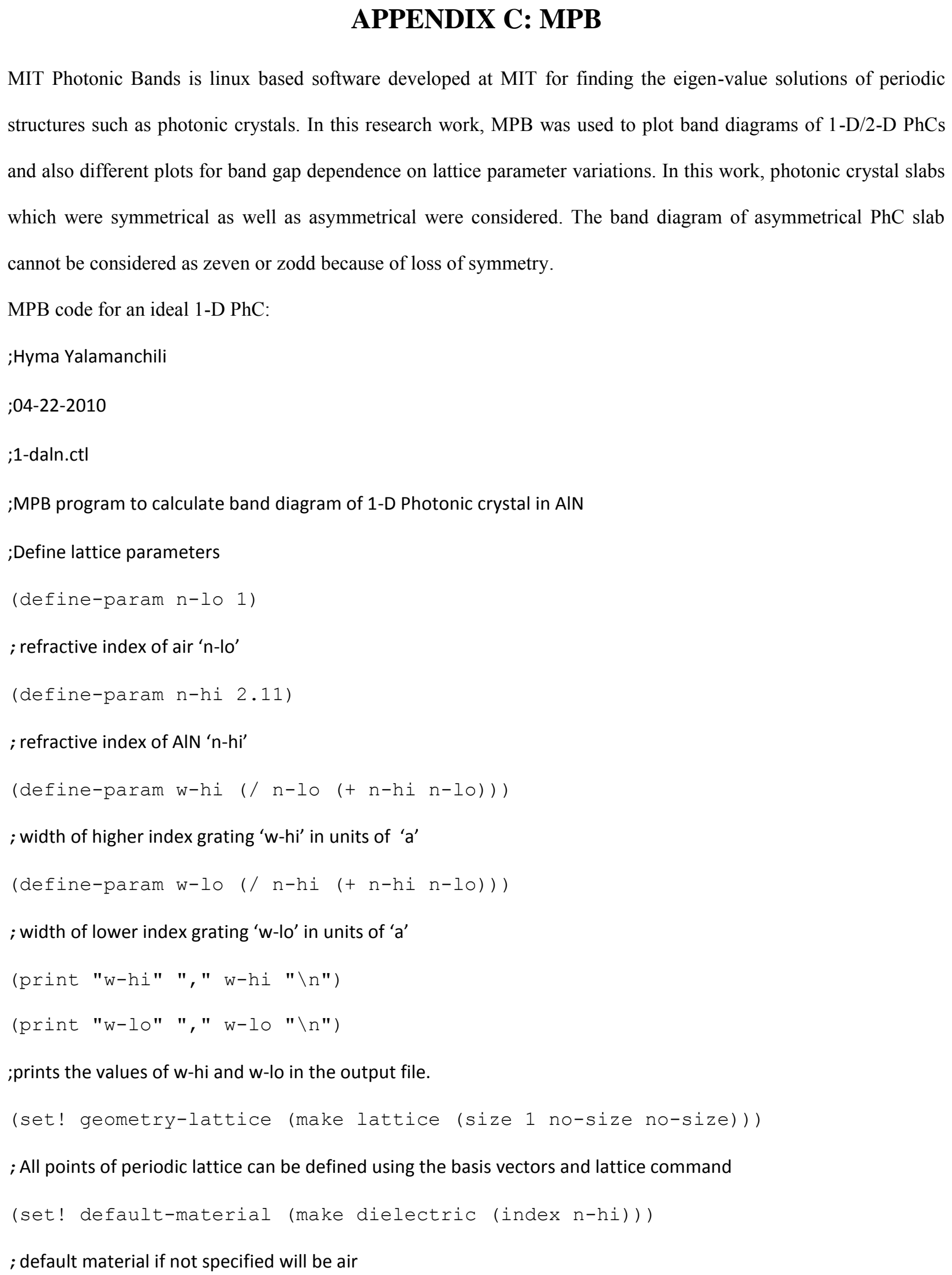

MIT Photonic Bands is linux based software developed at MIT for finding the eigen-value solutions of periodic structures such as photonic crystals. In this research work, MPB was used to plot band diagrams of 1-D/2-D PhCs and also different plots for band gap dependence on lattice parameter variations. In this work, photonic crystal slabs which were symmetrical as well as asymmetrical were considered. The band diagram of asymmetrical PhC slab cannot be considered as zeven or zodd because of loss of symmetry.

MPB code for an ideal 1-D PhC:

;Hyma Yalamanchili

;04-22-2010

;1-daln.ctl

;MPB program to calculate band diagram of 1-D Photonic crystal in AIN

;Define lattice parameters

(define-param n-lo 1)

; refractive index of air ' $n$-lo'

(define-param n-hi 2.11)

; refractive index of AIN ' $\mathrm{n}-\mathrm{hi}$ '

(define-param w-hi (/ n-lo (+ n-hi n-lo)))

; width of higher index grating 'w-hi' in units of 'a'

(define-param w-lo (/ n-hi (+ n-hi n-lo)))

; width of lower index grating ' $w$-lo' in units of ' $a$ '

(print "w-hi" "," $w-h i ~ " \backslash n ")$

(print "w-10" ", " $\mathrm{w}-10$ "\n")

;prints the values of w-hi and w-lo in the output file.

(set! geometry-lattice (make lattice (size 1 no-size no-size)))

; All points of periodic lattice can be defined using the basis vectors and lattice command

(set! default-material (make dielectric (index n-hi)))

; default material if not specified will be air 


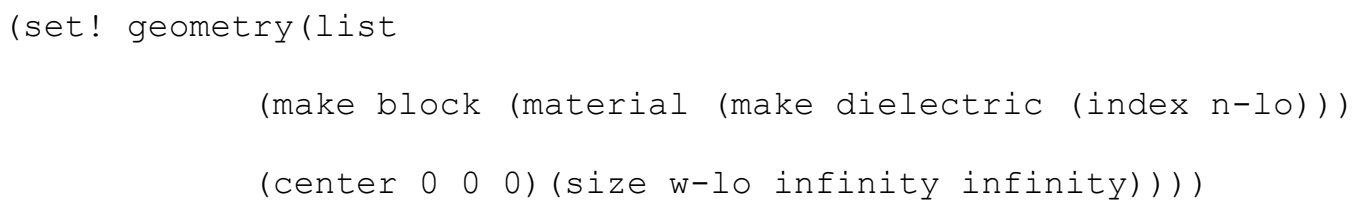

The commands for running the code and acquiring the dielectric structure can be found in (http://abinitio.mit.edu/mpb/).

For a Photonic crystal slab structure, after determining the lattice parameters and grating widths the thickness of the slab has to be specified.

(define-param h 0.8 )

; thickness of the slab

(define-param supercell-h 8)

; If thickness of slab has to be specified in MPB, a lattice vector has to be ;defined in that direction instead of ;specifying no-size. But, if a lattice size is defined then the structure repeats itself in that direction. Therefore, 
; a supercell-h is defined with a thickness of several wavelengths of light ; thick, allowing for negligible guided mode ;power existing outside of background. This supercell contains slab structure at the center.

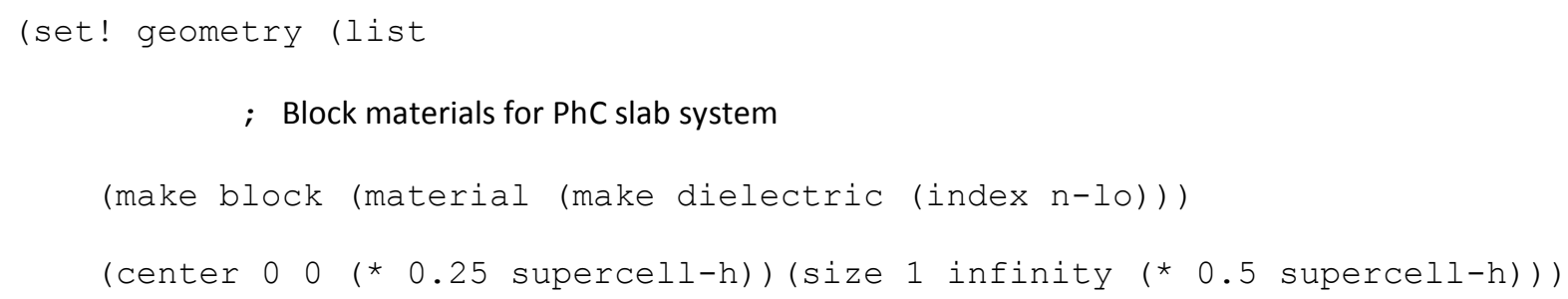

Once the simulations are performed and data is acquired from the output file, light cone for that structure has to be calculated . For a symmetric PhC slab, light cone can be determined by $\omega / c=k / n_{\text {cladding. }}$. This light cone separates the guided modes and radiative modes. The band gap which lies below the light cone can thus be determined from the zeven and zodd band diagram. For an asymmetrical PhC slab with different cladding layers, the light cone is an union of the light cones of two cladding layers. There will not be any bandgap in such structures due to absence of zeven and zodd modes. 


\section{APPENDIX D: ELLIPSOMETRY/Reflectivity}

Process flow for acquiring reflectance data using ellipsometer for mapping the experimental data to the theoretically drawn band diagram can be shown in Process flow below.

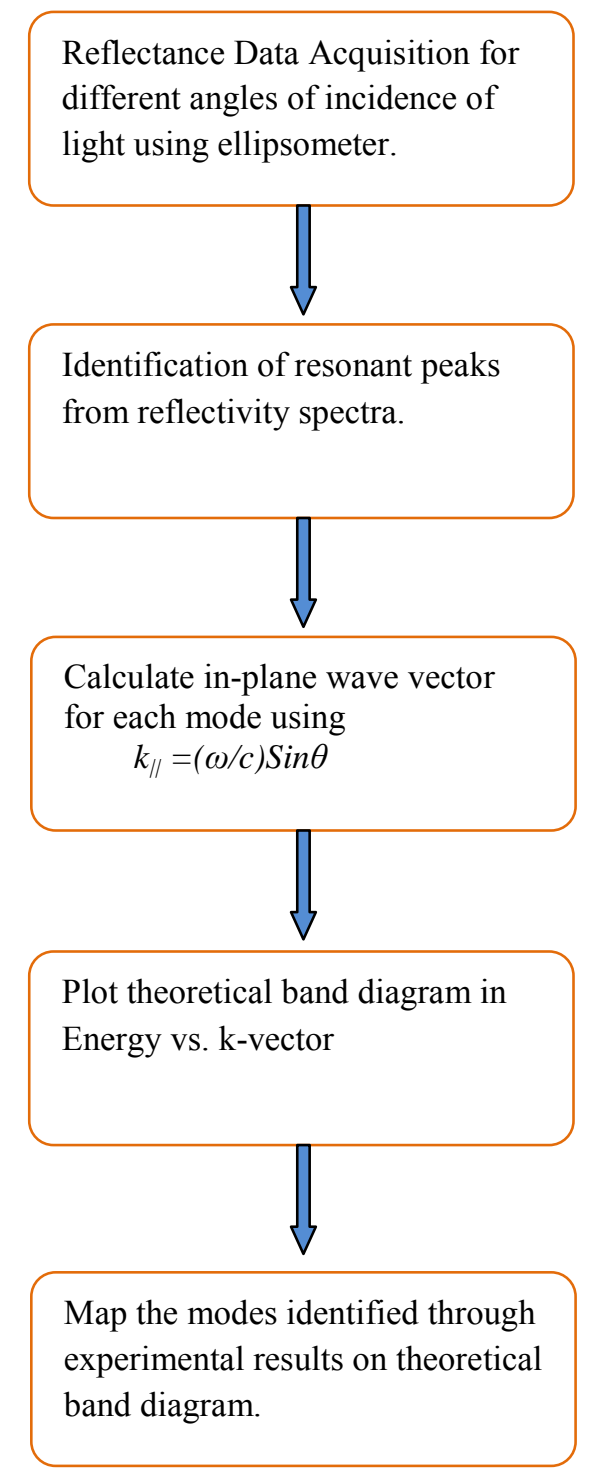

Figure(c) Process flow for mapping experimental data to theoretical band diagram

Ellipsometer uses a beam which has a width of $2-3 \mathrm{~mm}$ for data acquisition from the sample. But, the nanoscale PhC pattern size is approximately $600 \mu \mathrm{m}^{2}$. So, using a beam width of $3 \mathrm{~mm}$ would result in redundant data. Therefore, lenses were used to collimate the beam to a size of $\sim 500-600 \mu \mathrm{m}^{2}$. After the insertion of lenses into the input and output arms of ellipsometer, it needs alignment and calibration as specified in the ellipsometer manual.

1.Normal reflectance data can be acquired at different angles by following steps. 
Hardware $\rightarrow$ acquire data $\rightarrow \mathrm{R} \& \mathrm{~T}$ data $\rightarrow$ baseline scan and datascan, Refl p-pol without backside, specify angle (eg. $\left.70^{0}\right)$.

A base line data has to be acquired keeping the arms in $90^{\circ}$ angle position. After acquiring data at a specified angle, reflectance data can be acquired at different angles of incidence following the same steps as in (a). One baseline scan is sufficient for multiple angles of incidence. The data acquired at variable angular incidence is saved in a .dat file for further processing.

2.Resonance peaks or inflection points were identified from the reflectance graph plotted using MATLAB or ORIGIN or Excel.

3.Parallel component of k-vector for each peak identified can be calculated using $k_{\|}=(\omega / c) \operatorname{Sin} \theta$.

4.Theoretical band diagram calculated using MPB is not only plotted as frequency vs. k-wave vector but also it is normalized. Therefore, frequency band diagram is converted into Energy band diagram to match the ellipsometry data taking into consideration lattice constant.

5. MATLAB can be used to further map the experimental data to the theoretical band diagram. 


\section{APPENDIX E: ANSYS}

ANSYS is commercial software used for numerically solving a wide variety of mechanical structural analyses. FEA can be performed using Graphical user interface as well as command files.

Inorder to simulate a structure in GUI, the following steps are implemented.

1. Preferences $\rightarrow$ structural, electric $\rightarrow$ ok

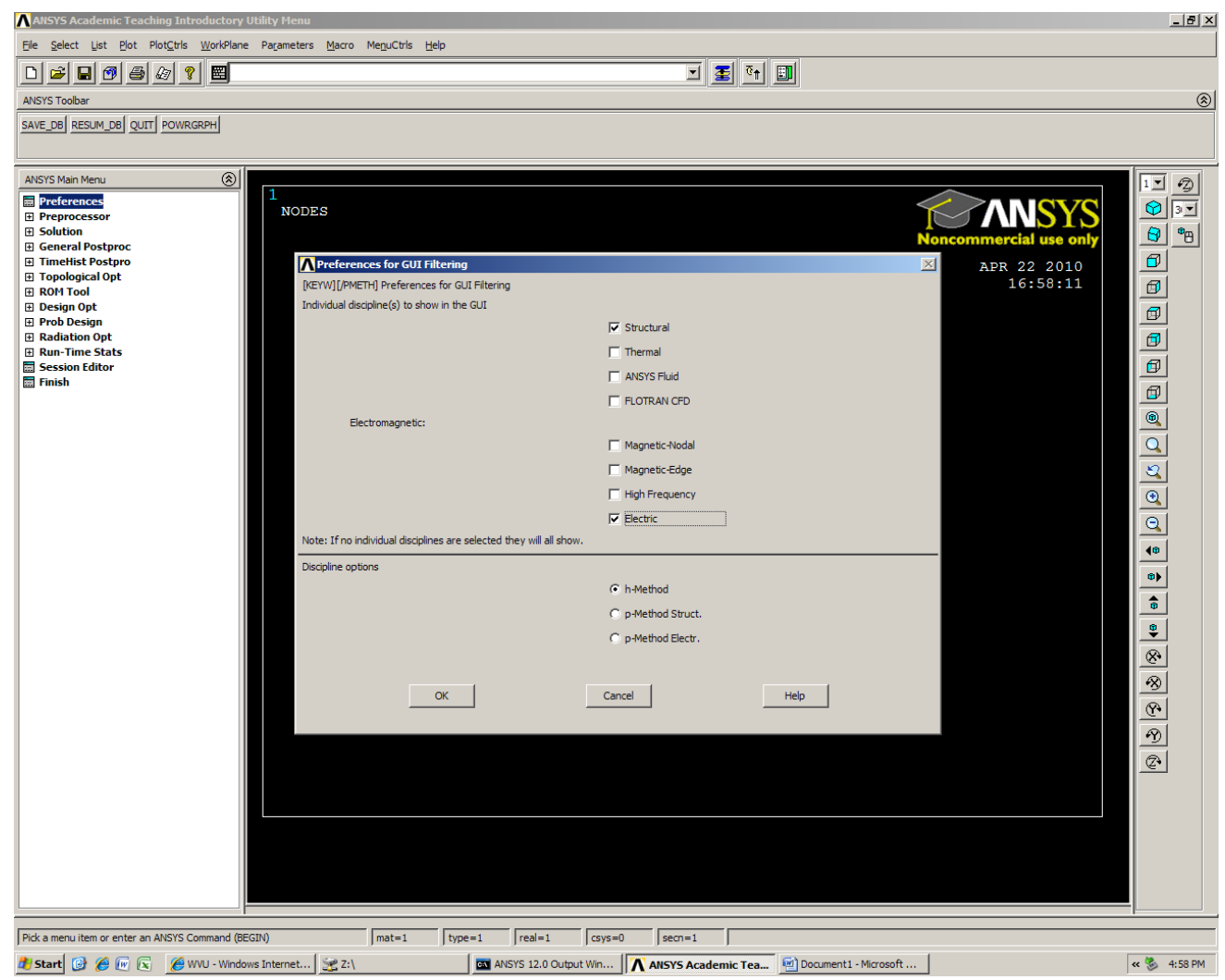

Figure(d) Coupled behavior of piezoelectric material can be analyzed by selecting structural/electric analysis

2. Preprocessor $\rightarrow$ Element type $\rightarrow$ Add/Edit/Delete $\rightarrow$ Add $\rightarrow$ coupledfield $\rightarrow$ scalar brick 5or Scalar Tet $98 \rightarrow$ ok

3. Element types $\rightarrow$ options $\rightarrow \mathrm{UX}, \mathrm{UY}, \mathrm{UZ}$, volt $\rightarrow$ ok

4. Preprocessor $\rightarrow$ Material Props $\rightarrow$ material models $\rightarrow$ Structural $\rightarrow$ Linear $\rightarrow$ Elastic $\rightarrow$ Anisotropic $\rightarrow$ insert compliance matrix. 


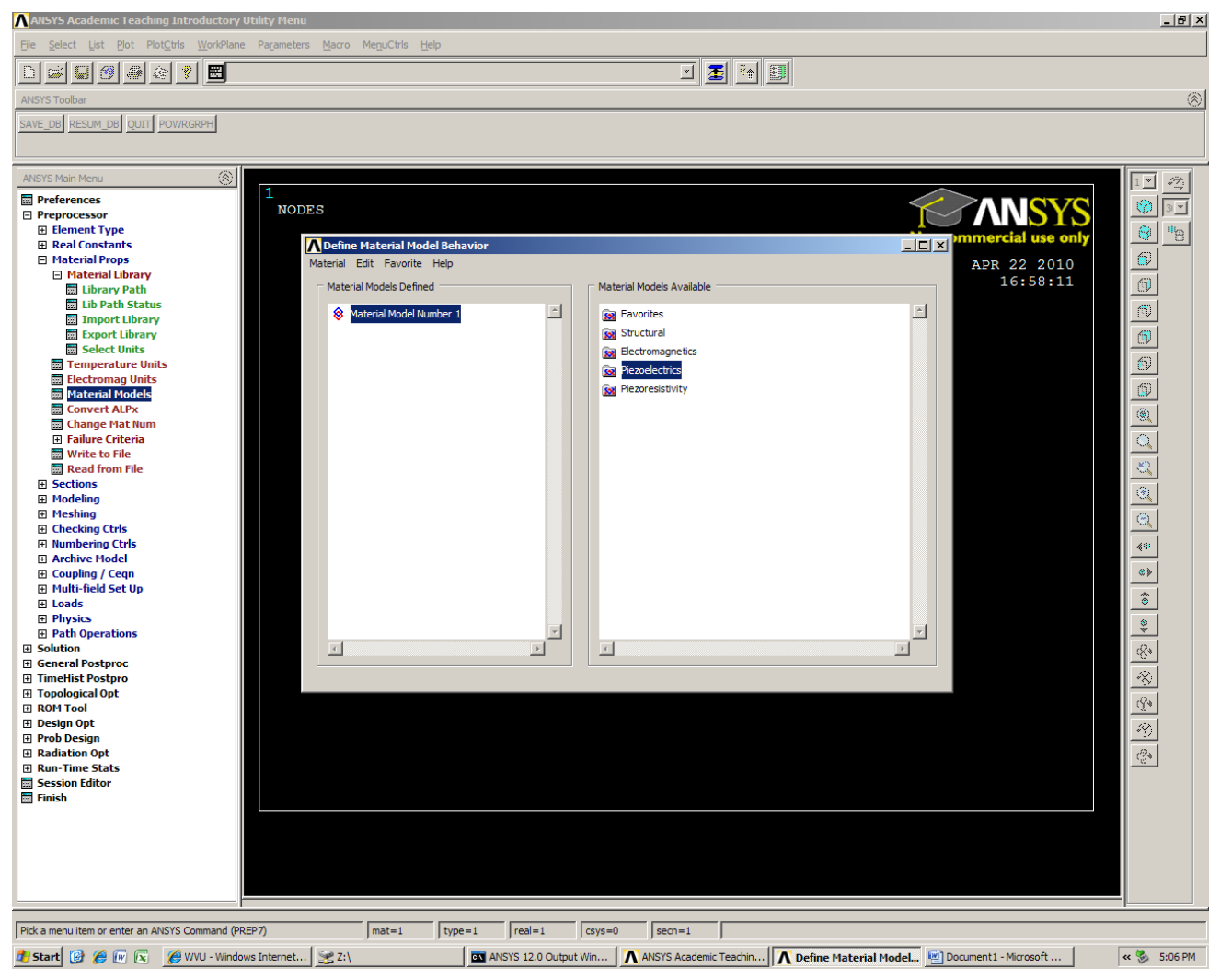

Figure(e) Material properties can be given in the material properties window

5. Preprocessor $\rightarrow$ Material Props $\rightarrow$ material models $\rightarrow$ Structural $\rightarrow$ Density $\rightarrow$ insert ok

6. Preprocessor $\rightarrow$ Material Props $\rightarrow$ material models $\rightarrow$ Structural $\rightarrow$ Electro-magnetics $\rightarrow$ relative permittivity $\rightarrow$ orthotropic $\rightarrow$ enter permittivity tensor value.

7. Preprocessor $\rightarrow$ Material Props $\rightarrow$ material models $\rightarrow$ Structural $\rightarrow$ Piezoelectrics $\rightarrow$ value

8. Preprocessor $\rightarrow$ modeling $\rightarrow$ create the structure using the drawing tools present.

9. Preprocessor $\rightarrow$ Meshing $\rightarrow$ Mesh attributes $\rightarrow$ picked volumes $\rightarrow$ select the volume to be meshed

10. Size of the mesh can be adjusted using size-ctrls button.

11. Preprocessor $\rightarrow$ mesh $\rightarrow$ volumes $\rightarrow$ select volumes $\rightarrow$ ok

12. After meshing the structure -boundary conditions can be applied using define loads option.

13. Solution $\rightarrow$ analysis type $\rightarrow \mathrm{DC} / \mathrm{AC} /$ Harmonic analysis

14. Solution $\rightarrow$ define loads - Apply $\rightarrow$ structural/Electric boundary conditions 


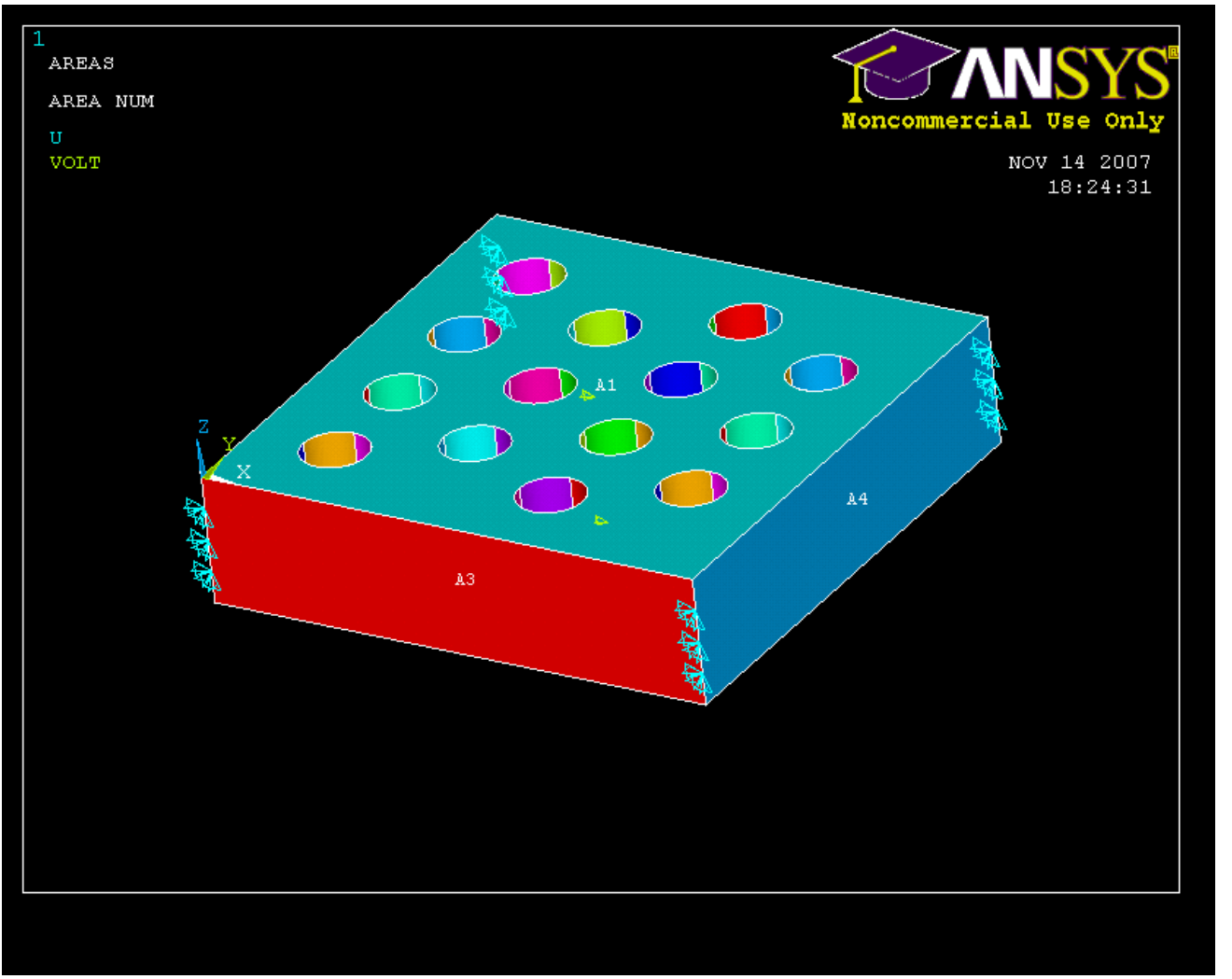

Figure(f) Zero displacement applied on the corners of the structure whereas voltage is applied on top and bottom plane

15. Solve $\rightarrow$ current LS

16. General post processor can be used to analyze results.

These are the basic steps used for a coupled field analysis of a piezoelectric material. After simulation, the results can be analyzed in general post processor. 


\section{Bibliography}

[ $\left.{ }^{1}\right] J$. D. Joannopoulos, R.D. Meade, and J.N. Winn, "Photonic Crystals: Molding the Flow of Light", (Princeton University Press, Princeton, 1995).

$\left.{ }^{2}\right]$ Daniel L. Barton and Arthur J. Fischer, "Photonic crystals improve LED efficiency", 2006 SPIE, The International Society for Optical Engineering.

$\left[{ }^{3}\right]$ Hatice Altug, Dirk Enlund and Jelenavu Ckovic "Ultrafast photonic crystal nanocavity laser", Published online: 1 July 2006; doi:10.1038/nphys343.

$\left[{ }^{4}\right]$ Chii-Chang Chen, Hung-Da Chien, and Pi-Gang Luan(20 November 2004), "Photonic crystal beam splitters", Applied optics, Vol. 43, No. 33.

$\left.{ }^{5}\right]$ Mads Brokner Christiansen(16 February 2009), "Polymer photonic crystal dye lasers as Optofluidic Cell Sensors", Optics Express 2722,Vol 17,No.4.

$\left.{ }^{6}\right]$ J. M. Dawson, J.R. Nightingale, R.P. Tompkins, S. Yeldandi, T.H. Myers, K. Jo, A. Timperman, D. Korakakis, X. Cao, and L.A. Hornak(2008), "Design and Characterization of Optofluidic Photonic Crystal Structures for the Detection of Fluorescent-Labeled Biomolecules, " to be published in Proc. 213th ECS Meeting, Phoenix, AZ, USA .

[7]. J.M. Dawson, J.R. Nightingale, R.P. Tompkins, X. Cao, T.H. Myers, L.A. Hornak, and D. Korakakis(2008), “GaN Photonic Crystal-Based, Enhanced Fluorescence Biomolecule Detection System,” Mater. Res. Soc. Symp. Proc., 1040, 1040-Q09-29.

$\left.{ }^{8}\right]$. J.R.Nightingale(2008), "Optical Biosensors: SPARROW Biosensor and Photonic-crystal based biosensor Flouroscene Enhancement,” Master's Thesis, Lane Dept of Comp.Sci and Elec.Engr.,West Virginia University, Morgantown.

[']. Y. Saado, M. Golosovsky, D. Davidov, and A. Frenkel(2002), "Tunable photonic band gap in self-assembled clusters of floating magnetic particles,” Phys. Rev. B 66, 195108-195113.

$\left[{ }^{10}\right]$ J. J. Zhou, C.Q. Sun, K. Pita, Y.L. Lam, Y. Zhou, S.L. Ng, C.H. Kam, L.T. Li, and Z.L. Gui (2001), ”Thermally tuning of the photonic band gap of $\mathrm{SiO} 2$ colloid-crystal infilled with ferroelectric BaTiO3,” Appl. Phys. Lett. 78, 661-663.

$\left[{ }^{11}\right]$. H. Nemec, P. Kuzel, L. Duvillaret, A. Pashkin, M. Dressel, and M. T. Sebastian, "Highly tunable photonic crystal filter for the terahertz range," Optics Letters, 30 (5), 549,2005. 
$\left.{ }^{12}\right]$ S. Savel'ev, A.L. Rakhmanov, and F. Nori, “Josephson vortex lattices as scatterers of terahertz radiation: Giant magneto-optical effect and Doppler effect using terahertz tunable photonic crystals," Phys. Rev. B, 74, 184512 (2006).

$\left[{ }^{13}\right]$ L. Feng, P. Liu, Y. F. Tang, Y.-F. Chen, J. Zi, S.-N. Zhu, and Y.-Y. Zhu, "Tunable negative refraction in a two dimensional active magneto-optical photonic crystal”, Phys. Rev. B, 71, 195106 (2005).

$\left[{ }^{14}\right]$ Shengli Pu, Tao Geng, Xianfeng Chen, Xianglong Zeng, Ming Liu, and Ziyun Di, “Tuning the band gap of selfassembled superparamagnetic photonic crystals in colloidal magnetic fluids using external magnetic fields", Journal for Magnetism and Magnetic materials, Volume 320,Issue 19, Oct 2008, pp:2345-2349.

$\left[{ }^{15}\right]$ L. Lalouat, B. Cluzel, P. Velha, E. Picard, D. Peyrade, J.P. Hugonin, P. Lalanne, E. Hadji, and F. de Fornel, "Near-field interactions between a subwavelength tip and a small-volume photonic-crystal nanocavity," Phys. Rev $B, 76,041102(\mathrm{R})(2007)$.

$\left[{ }^{16}\right]$ I. Märki, M. Salt, and H.P. Herzig, “Tuning the resonance of a photonic crystal microcavity with an AFM probe”,Optics Express, 14 (7), 2969 (2006).

$\left[{ }^{17}\right]$. T.G. Euser, H. Wei, J. Kalkman, Y. Jun, A. Polman, D.J. Norris, and W.L. Vos, "Ultrafast optical switching of three-dimensional Si inverse opal photonic band gap crystals," J. Appl. Phys., 102, 053111 (2007).

$\left[{ }^{18}\right]$ A.Z. Garcia-Déniz, P. Murzyn, A.M. Fox, D.O. Kundys, J.-P.R. Wells, D.M. Whittaker, M.S. Skolnick, T.F. Krauss, and J.S. Roberts, "Ultrafast reflectivity modulation in $\mathrm{Al}_{\mathrm{x}} \mathrm{Ga}_{1-\mathrm{x}} \mathrm{As} / \mathrm{In}_{\mathrm{y}} \mathrm{Al}_{\mathrm{x}} \mathrm{Ga}_{1-\mathrm{x}-\mathrm{y}} \mathrm{As}$ multiple quantum well photonic crystal waveguides," Phys. Rev. B, 74, 165327 (2006).

$\left[{ }^{19}\right]$ I. Märki, M. Salt, H.P. Herzig, R. Stanley, L. El Melhaoui, P. Lyan, and J.M. Fedeli, "Optically tunable microcavity in a planar photonic crystal silicon waveguide buried in oxide," Optics Letters, 31 (4), 513 (2006).

$\left.{ }^{20}\right]$. M.W. Lee, C. Grillet, C.L.C. Smith, D.J. Moss, B.J. Eggleton, D. Freeman, B. Luther-Davies, S. Madden, A. Rode, Y. Ruan, Y. Lee, "Photosensitive post tuning of chalcogenide photonic crystal waveguides," Optics Express, 15 (3), 1278 (2007).

$\left[{ }^{21}\right]$. P. Halevi, J.A. Reyes-Avendaño, and J.A. Reyes-Cervantes, "Electrically tuned phase transition and band structure in a liquid-crystal-infilled photonic crystal,” Phys. Rev. E, 73, 040701(R) (2006).

$\left.{ }^{22}\right]$. G. Alagappan, X.W. Sun, P. Shum, M.B. Yu, and M.T. Doan, "One-dimensional anisotropic photonic crystal with a tunable bandgap," J. Opt. Soc. Am. B, 23 (1), 159 (2006). 
${ }^{23}$ ]. D.M. Pustai, A. Sharkawy, S. Shi, and D.W. Prather, “Tunable photonic crystal microcavities," Applied Optics, 41 (26), 5574 (2002).

$\left.{ }^{24}\right]$. M Ghulinyan, Z. Gaburro, D.S. Wiersma, and L. Pavesi, "Tuning of resonant Zener tunneling by vapor diffusion and condensation in porous optical superlattices," Phys. Rev B, 74, 045118 (2006).

$\left[{ }^{25}\right]$. M. Notomi and S. Mitsugi, "Wavelength conversion via dynamic refractive index tuning of a cavity," Phys. Rev. A, 73, 051803(R) (2006).

$\left[{ }^{26}\right]$. T. Takahata, K. Hoshino, K. Matsumoto, I. Shimoyama, "Photonic crystal tuned by cantilever", 18th IEEE International Conference on Micro Electro Mechanical Systems, Miami, Florida, USA, (2005).

$\left[{ }^{27}\right]$. W. Park and J.-B. Lee, "Mechanically tunable photonic crystal structure," Appl. Phys. Lett., 85 (21), 4845 (2004).

$\left[{ }^{28}\right]$. E. Bormashenko, R. Pogreb, O. Stanevsky, Y. Biton, Y. Bormashenko, V. Streltsov, and Y. Socol, "2D photonic crystals deposited on polymer piezoelectric substrates - new kind of MOEMS," in Proc. of the 11th IEEE International Conference on Electronics, Circuits and Systems (ICECS 2004), 459, (2004).

$\left[{ }^{29}\right]$. S. Kim and V. Gopalan(2001), "Strain-tunable photonic band gap crystals,” Appl. Phys. Lett. 78, 3015-3017 .

$\left.{ }^{30}\right]$ P.A. Bermel and M. Warner(2002),"Photonic band structure of highly deformable self-assembling systems," Phys.Rev. E 65, 10702-10705.

$\left[{ }^{31}\right.$ ]. G. Alagappan, X.W. Sun, P. Shum, M.B. Yu, and M.T. Doan(2006), "One-dimensional anisotropic photonic crystal with a tunable bandgap,” J. Opt. Soc. Am. B, 23 (1), 159.

$\left[{ }^{32}\right]$.Chee Wei Wong,Xiaodong Yang,Peter T Rakich,Steven G.Johnson(2004), “Strain-Tunable Photonic BandGap Microcavity waveguides in Silicon at 1.55um,"'Proceedings of SPIE Vol.5511,SPIE,Bellingham,WA,236-239

$\left[{ }^{33}\right]$ Y. Benachour and N. Paraire (2007), "Characterization of planar photonic crystals using surface coupling techniques at large wavelengths," Chinese optics letters, Vol. 5, No. 9.

$\left[{ }^{34}\right]$ Vincent.R. Pagan, “Aluminum Nitride deposition/ characterization \& PMEMS/ SAW Device Simulation/ Fabrication”, LCSEE, Morgantown, WV, 2009.

$\left[{ }^{35}\right]$ Bernard Jaffe, William R.Cook, "Piezoelectric Ceramics: Principles and Applications”, APC International. Ltd, 2002. 
$\left.{ }^{36}\right]$ Humberto. C, "Thin-film bulk acoustic wave resonators: FBAR, Fabrication, heterogeneous Integration with CMOS Technologies and sensor Applications", PhD Thesis, Univ. of Barcelona.

$\left[{ }^{37}\right]$ Cady W.G, "Piezoelectricity”, Mc GrawHill, New York ,1946.

${ }^{38}$ R.G. Hunsperger, “Integrated Optics: Theory and Technology”, 2nd Edition, Springer-Verlag publications.

${ }^{39}$ P. Samudrala, “Alumina Waveguide Characterization and SPARROW Biosensor Modeling," Master's thesis, Lane Dept. of Comp. Sci. and Elec. Engr., West Virginia University, Morgantown, WV, 2006.

$\left[{ }^{40}\right]$ http://ab-initio.mit.edu/wiki/index.php/MIT_Photonic_Bands

$\left[{ }^{41}\right]$ Hojo Hitoshi and Mase Atsushi, "Dispersion relation of Electomagnetic waves in one-dimensionsl plasma photonic crystals," Rapid Communications ,2004.

$\left.{ }^{42}\right]$ S. Shi, C. Chen, and D. W. Prather, "Plane-wave expansion method for calculating band structure of photonic crystal slabs with perfectly matched layers," J. Opt. Soc. Am. A 21, 1769-1775, 2004.

$\left[{ }^{43}\right]$ http://www.mece.ualberta.ca/tutorials/ansys/

$\left[{ }^{44}\right]$ Randy. P.Tompkins, J. M.Dawson, J. Nightingale, S. Yeldandi, H. Yalamanchili, B.Hamza, “ Polarity Inverted GaN for photonic crystal Biosensor Applications", 214 ${ }^{\text {th }}$ ECS Meeting, 2008.

$\left[{ }^{45}\right]$ Steven.G.Johnson, "Photonic Crystals: From theory to practice”, Doc .of. Phil Thesis, MIT,2001.

$\left.{ }^{46}\right]$ Chee Wei Wong,Xiaodong Yang,Peter T Rakich,Steven G.Johnson, "Strain-Tunable Photonic BandGap Microcavity waveguides in Silicon at 1.55um,"Proceedings of SPIE Vol.5511,SPIE,Bellingham,WA,236-239, 2004.

$\left[{ }^{47}\right.$ ]. Y. K. Sin and K. Ibrahim"2D Silicon-based Photonic Crystals",ICSE2006 Proc. 2006, Kuala Lumpur, Malaysia,2006.

$\left[{ }^{48}\right]$. V.A.Tolmachev, T.S.Perova,E.V Astrova,B.Z.Volchek, and J.K.Vij, "Vertically etched Silicon as 1D Photonic crystal," phys.stat.sol. (a)197, No.2,544-548,2003.

$\left[{ }^{49}\right]$. Jae Soong I,Yeonsang Park and Heonsu Jeon ,“Optimal Design for one dimensional photonic crystal,” Journal of Lightwave Technology, Vol 22,No.2,509-513,2004.

$\left[{ }^{50}\right]$ K. Lee, H.Yalamanchili, J.Dawson, D.Korakakis, "Fabrication of Two Dimensional Array of Silica Nanospheres on GaN using Spin Coating Method and Nomarski Image Processing”, Mat. Res.Soc,Vol 1207,2009.

$\left[{ }^{51}\right]$ S. Y. Lin and E. Chow, "Direct measurement of quality factor in a two dimensional photonic crystal microcavity," Optics Letters, 26 (2001).

$\left[{ }^{52}\right]$ V. A. Tolmachev, Optics and Spectroscopy, 97, 276 (2004). 
$\left[{ }^{53}\right]$ C. Jiang and T. Jiyu, "Optical properties in $1 \mathrm{D}$ photonic crystal structure using $\mathrm{Si} / \mathrm{C}_{60}$ multilayers," $J$. Semiconductors, 30 (2009).

$\left[{ }^{54}\right]$ M. Akihikoshinya, Optical and Quantum Electronics, 34, 113 (2002).

$\left[{ }^{55}\right]$ Optiwave Documention for OptiBPM 8.0, Optiwave Corporation, Ottawa, ON, Canada.

$\left[{ }^{56}\right]$ K. M. Taylor and Camilie Lenie, "Some Properties of Aluminum Nitride", Journal of the Electrochemical Society, Apr. (1960), pp. 308-314.

$\left[{ }^{57}\right]$ C. R. Aita, C. J. Gawlak," The dependence of aluminum nitride film crystallography on sputtering plasma composition” Journal of Vacuum Science Technology A, 1, (1983), 403

$\left[{ }^{58}\right]$ I. Cimalla, Ch. Foerster, V. Cimalla, V. Lebedev, D. Cengher, and O. Ambacher, "Wet chemical etching of AlN in KOH solution", phys. stat. sol. (c) 3, No. 6, (2006),pp:1767-1770.

$\left[{ }^{59}\right]$ D. Zhuang, J. H. Edgar,B. Strojek, J. Chaudhuriand and Z. Rek, "Defect-selective etching of bulk AlN single crystals in molten KOH/NaOH eutectic alloy", Journal of Crystal Growth ,262 (2004) ,pp 89-94.

$\left[{ }^{60}\right]$ J. R. Mileham, S. J. Pearton, C. R. Abernathy, J. D. MacKenzie,", Wet chemical etching of AlN", Appl. Phys. Letters. 67, (1995), pp:1119.

$\left[{ }^{61}\right]$ R. J. Shul, G. A. Vawter, C. G. Willison, M. M. Bridges, J. W. Lee, S. J. Pearton, C. R. Abernathy, ”Comparison of plasma etch techniques for III-V nitrides”, Solid State Electronics, 42, 12 ,(1998),pp: 2259-2267.

$\left[{ }^{62}\right]$ Kuo-Sheng Kao, Chung-Jen Chung, Ying-Chung Chen, Tien-Fan Ou, Tai-Kang Shing, ” The influence of varied sputtering condition on piezoelectric coefficients of AIN thin films", IEEE Internations Ultrasonics, Ferroelectrics, and Frequency Control Joint 50th Anniversary Conference, 2004.

$\left[{ }^{63}\right]$ Gary Keith Fedder, "Simulation of Micro electro mechanical systems", PhD Thesis, University of Berkeley, 1994.

$\left[{ }^{64}\right]$ Niels Tasy, Tonny Sonnenberg, Henri Jansen, Rob Legtenberg and Miko Elwenspoek, "Stiction in surface micromachining", J. Micromech. Microeng, (1996) pp.385-397

$\left[{ }^{65}\right]$ Zhao Yapu, "Stiction and anti-stiction in NEMS and MEMS", Chinese Society of Theo. and Appl. Mechanics, Vol 19, No.1, February 2003.

$\left[{ }^{66}\right]$ Z.Suo, "Fracture in thin films", Prepared for Encyclopedia of Materials: Science and Technology, Second Edition, Elsevier Science, 2001. 
$\left[{ }^{67}\right]$ Si-Hyung Lee,Ki Hyun Yoon, Deok-Soo Cheong, Jeon-Kook Lee, "Relationship between residual stress and structural properties of AIN films deposited by r.f. reactive sputtering”, Thin Solid Films ,435 (2003) ,pp:193-198 $\left[{ }^{68}\right]$ Shinya, A, Mitsugi, S, Kuramochi, and Notomi, "Ultrasmall multi-port channel drop filter in two-dimensional photonic crystal on silicon-on-insulator substrate", Opt. Express 14, 12394-12400 (2006).

$\left[{ }^{69}\right]$ Sugimoto, Y, Tanaka, Ikeda, Nakamura, H, Kanamoto, Kohkouchi, Watanabe, Y, Inoue, K. and Asakawa, K, "Fabrication and characterization of photonic crystal-based symmetric Mach-Zehnder (PC-SMZ) structures based on GaAs membrane slab waveguides”, IEEE J. Sel. A. Comm. 23, 1308-1314 (2005).

$\left[{ }^{70}\right]$ C. Sibili, T.M. Benson, M. Marciniak, T. Szoplik "Photonic crystals: Physics and Technology", Springer Verlag, 2008.

$\left[{ }^{71}\right]$ Labilloy, D, Benisty, Weisbuch, Krauss, DeLaRue. R.M, Bardinal.V, Houdre,R, Oesterle.U, Cassagne. D, and Jouanin.C, "Quantitative measurement of transmission, reflection, and diffraction of two-dimensional photonic band gap structures at near-infrared wavelengths", Phys. Rev. Lett. 79, pp: 4147-4150, (1997).

$\left.{ }^{72}\right]$ V.N Astratov, R.M. Stevenson, I.S. Culshaw, D.M. Whittaker, M.S. Skolnick, T.F. Krauss, and R.M. De La Rue, "Heavy photon dispersion in Photonic crystal waveguides", Appl. Phys. Lett. 77, 178-180 (2000).

$\left.{ }^{73}\right]$ V.N. Astratov, I.S. Culshaw, R.M. Stevenson, D.M. Whittaker, M.S. Skolnick, T.F. Krauss, and R.M. DeLaRue, "Resonant Coupling of Near-Infrared Radiation to Photonic Band Structure Waveguides", J. of Lightwave Technology, v.17, 2050-2057 (1999).

$\left[{ }^{74}\right]$ V.N. Astratov, D.M. Whittaker, I.S. Culshaw, R.M. Stevenson, M.S. Skolnick, T.F. Krauss, and R. M.DeLaRue, "Photonic Band Structure Effects in the Reflectivity of Periodically Patterned Waveguides", Phys. Rev. B 60, R16255-16258 (1999).

$\left[{ }^{75}\right]$ M. Patrini, M. Galli, F. Marabelli, M. Agio, L. C. Andreani, D. Peyrade, and Yong Chen, "Photonic Bands in Patterned Silicon-on-Insulator Waveguides", IEEE journal of quantum electronics, Vol. 38, No. 7, July 2002.

$\left[{ }^{76}\right]$ Y. Benachour and N. Paraire, "Characterization of planar photonic crystals using surface coupling techniques at large wavelengths,"Chinese optics letters, Vol. 5, No. 9 (2007).

$\left[{ }^{77}\right]$ http://www.piketech.com/products/product-documentation-pdfs/VeeMAX_PDS.pdf

$\left[{ }^{78}\right]$ J. A Woollam, B. Johs, C. Herzinger, "Overview of variable angle spectroscopic ellipsometry (VASE), part I: Basic theory and typical applications", Critical Reviews of Optical Science and Technology. Vol CR72, 3-28 (2000). 
$\left[{ }^{79}\right]$ http://deeea.urv.cat/DEEEA/cen2008/docs/Moreno_UMH_01.pdf

$\left[{ }^{80}\right]$ M. Qiu, "Band gap effects in asymmetric photonic crystal slabs," Physics Review B 66, 033103 (2002).

$\left[{ }^{81}\right]$ Erwin. G. Loewen, Evgeny Popov, "Diffraction gratings and Applications”, Marcel Dekker Inc.,1997.

$\left[{ }^{82}\right]$ John. M. Jarem, Partha. P. Banerjee, “Computational methods for electromagnetic and optical systems”, Marcel Dekker Inc., 2000.

$\left[{ }^{83}\right]$ N. Paraire, Y. , "Investigation of planar photonic crystal band diagrams under the light cone using surface coupling techniques", Appl.Phys , 245-250, (2007). 